\title{
Dynamical System Analysis of Ignition Phenomena Using the Tangential Stretching Rate Concept
}

\author{
Mauro Valorani $^{\mathrm{a}}$, Samuel Paolucci ${ }^{\mathrm{b}}$, Emanuele Martellic, Temistocle Grenga ${ }^{\mathrm{b}}$, Pietro Paolo Ciottoli ${ }^{\mathrm{a}}$ \\ ${ }^{a}$ Mechanical and Aerospace Engineering Dpt., Sapienza University of Rome, Rome, Italy \\ ${ }^{b}$ Mechanical and Aerospace Engineering Dpt., University of Notre Dame, South Bend, Indiana, USA \\ ${ }^{c}$ Industrial and Information Engineering Dpt., Second University of Naples, Caserta, Italy
}

\begin{abstract}
We analyze ignition phenomena by resorting to the stretching rate concept formerly introduced in the study of dynamical systems. We construct a Tangential Stretching Rate (TSR) parameter by combining the concepts of stretching rate with the decomposition of the local tangent space in eigen-modes. The main feature of the TSR is its ability to identify unambiguously the most energetic scale at a given space location and time instant. The TSR depends only on the local composition of the mixture, its temperature and pressure. As such, it can be readily computed during the post processing of computed reactive flow fields, both for spatially homogeneous and in-homogenous systems.

Because of the additive nature of the TSR, we defined a normalized participation index measuring the relative contribution of each mode to the TSR. This participation index to the TSR can be combined with the mode amplitude participation Index of a reaction to a mode - as defined in the Computational Singular Perturbation (CSP) method - to obtain a direct link between a reaction and TSR. The reactions having both a large participation index to the TSR and a large CSP mode amplitude participation index are those contributing the most to both the explosive and relaxation regimes of a reactive system. This information can be used for both diagnostics and for the simplification of kinetic mechanisms.

We verified the properties of the TSR with reference to three nonlinear planar models (one for isothermal branched-chain reactions, one for a non-isothermal, one-step system, and for non-isothermal branched-chain reactions), to one planar linear model (to discuss issues associated with non-normality), and to test problems involving hydro-carbon oxidation kinetics.

We demonstrated that the reciprocal of the TSR parameter is the proper characteristic chemical time scale in problems involving multi-step chemical kinetic mechanisms, because (i) it is the most relevant time scale during both the explosive and relaxation regimes, and (ii) it is intrinsic to the kinetics, that is, it can be identified without the need of any ad-hoc assumption.
\end{abstract}

Keywords: Ignition, Chemical Kinetics, Computational Singular Perturbation, Tangential Stretching Rate

\section{Introduction}

Ignition of hydrocarbon (and hydrogen) fuels is controlled by branched-chain reactions and its complexity increases with the length/size of the fuel molecule.

The Computational Singular Perturbation (CSP) Method has been used to analyze two-stage ignition of n-heptane by Kazakov, et al. [1] and Goussis, et al. [2, 3]. They observed the occurrence in spatially homogeneous systems of two branches of positive eigenvalues during both chain-branching and thermal ignition. Chen, et al. [4] resorts to CSP to study high temperature ignition and combustion enhancement by dimethyl ether addition to methane-air mixtures. Lu, et al. [7-10] proposed an explosion index for Chemical Explosive Modes (CEM) to analyze DNS datasets of turbulent flames. Lu, et al. [11] also discussed the role of eigenvalues with a positive real part in the context of limit phenomena in combustion. Najm, et al., [12], found explosive modes in edge flame data sets. Gupta, et al. adopted CSP to classify ignition regimes in HCCI combustion [5], and n-heptane auto-ignition characteristics in DNS datasets [6]. All these ignition studies stress the role of eigenvalues with a positive real part in ignition and other limit phenomena for all fuels tested (hydrogen and hydrocarbons).

The present work analyzes the kinetic behavior related to ignition events by adopting the concept of local stretching rates introduced in [13]. The local stretching rates find their theoretical justification in the theory of normal hyperbolicity applied along system orbits/trajectories.

Upon close analysis, we found that the basic definition of the local stretching rate given in [13] fails to provide meaningful information when the trajectory proceeds through a region of complex eigenvectors. To overcome this shortcoming, we propose in this paper a modification of the local Tangential Stretching Rate (TSR) definition, which involves combining the eigen-decomposition of the Jacobian matrix of the vector field with the basic definition of the local TSR given in [13].

The main result of this work is the recognition that the TSR can be computed as a weighted average of the modulus of the eigenvalues of the problem, with weights that depend on (i) the (square of) mode amplitudes, and (ii) the degree of co-linearity between each eigenvector and the vector field.

In addition, because of its additive nature, we introduce a nor- 
malized index measuring the relative contribution of each mode to the TSR amplitude. This index can be further combined with the standard participation index of a reaction to a mode amplitude - as defined in CSP - to obtain a direct link between reactions and the TSR. This information can be used for both diagnostics and for the simplification of kinetic mechanisms.

We explore ignition with a planar (2D) model of a spatially homogeneous system according with an isothermal branchedchain explosion model proposed by Williams [20]. We analyze Williams' model on the basis of the CSP and TSR theories. Similar to what is found in detailed kinetic systems, Williams' model develops one pair of eigenvalues, both real and complex, with a positive real part during the explosive regime of ignition.

Our findings show that the TSR is able to track the most energetic scale(s) at all times, and that its own time evolution is smooth.

We carry out the TSR analysis of a planar (2D) model of thermal explosion model - firstly proposed by Semenov in [33, 34] to study the non-isothermal explosion process, which might occur in a reservoir where combustion generates heat released to the ambient through non-adiabatic walls. The Semenov system features an exponential non linearity.

We finally consider a planar (2D) model of a spatially homogeneous system for a non-isothermal branched-chain explosion model proposed by Kapila [22]. The main motivation to analyze this system is to assess the role of the underlying nonlinearities characterizing this model problem. In fact, Williams' model features a polynomial (quadratic) non linearity, whereas Kapila's model combines the exponential dependence of the temperature with the quadratic non linearity of the branchingchain reaction.

The critical comparison of the Williams, Semenov, and Kapila models allowed us to learn about the influence of the nature of the nonlinearities on the qualitative evolution of ignition.

The qualitative results obtained in the analysis of the kinetics of three fuels (methane [18], propane and n-heptane [19]) are very similar to those observed in the Williams model, this suggesting that the dominant behavior is controlled by the polynomial nonlinearities typical of branched-chain reactions. This finding is consistent with the results obtained by $\mathrm{Lu} \mathrm{[7-10]} \mathrm{and}$ Goussis [2,3] in their (independent) analyses.

We will show on the basis of the TSR analysis that during ignition, events are controlled not only by the two modes with positive real parts but also by a few modes having negative real parts. This finding extends what has been already assessed in the literature (e.g., [1-3, 7-10]) about the role of modes with positive real part.

While carrying out the TSR analysis of the hydrocarbon system, we observed the TSR to occasionally exhibit significant overshoots with respect to the underlying eigenvalues. We were eventually able to attribute this behavior to the development of nearly co-linear eigenvalues, which occurs when the Jacobian matrix becomes non-normal. To explore in depth this issue, we designed a planar linear system with tunable non-normality, and we analyzed its dynamics with the TSR index.

We found that non-normality is a necessary condition to gen- erate the overshoots, but not sufficient: overshoots of the TSR index manifest only along trajectories exhibiting a strong curvature in non-normal systems.

Finally, the combination of the participation index of reactions to modes and the participation index of modes to the TSR allows us to identify the most important reactions controlling the ignition in the three fuels considered. Among other things, this provides information on the reactions and species that are common in the ignition of the three fuels.

The paper is organized as follows. The theoretical derivation that leads to the definition of the TSR is presented in Sec. 2. The derivation of the equation for the vector dynamics is offered in 2.1. The derivation of the equation for the vector norm dynamics is obtained in 2.2. In Sec. 3, the Williams model is presented and discussed in light of both the CSP and TSR analyses, where we also introduce a two-dimensional definition of the TSR. The definition of the TSR is extended to the $N$ dimensional case in Sec. 4. The Participation Index of a mode to the TSR is introduced in Sec. 5. The TSR analysis of the Semenov model is in 7. The non-isothermal system by Kapila is defined in Sec. 8, and analyzed in Sec. 9. The outcome of the TSR analysis applied to the auto-ignition of three hydrocarbon fuels is illustrated in Sec. 10. Finally, issues connected with non-normality of the Jacobian are discussed in Sec. 11.

In Appendix A, we provide the detail of the implementation of the TSR when complex eigenvalues are present.

\section{Theory}

Consider a chemical kinetic system whose dynamics is described by the a Cauchy problem of the form:

$$
\frac{d \boldsymbol{x}}{d t}=\boldsymbol{g}(\boldsymbol{x}), \quad \boldsymbol{x}(0)=\boldsymbol{x}_{0}, \quad \boldsymbol{x} \in \mathbb{R}^{N} .
$$

The state vector $\boldsymbol{x}$ can be identified with the species concentration vector, the vector field $\boldsymbol{g}(\boldsymbol{x})=S \mathbf{r}(\boldsymbol{x})$ with the species reaction rate vector, $S$ with the stoichiometric coefficients matrix, $\mathbf{r}(\boldsymbol{x})$ with the net reaction rates vector, and $\boldsymbol{x}_{0}$ with the initial concentrations vector.

The definition of the state vector $\boldsymbol{x}$ can be extended to include thermo-dynamic variables (temperature, pressure, internal energy, entropy, ...), this requiring a suitable generalization of the coefficients matrix, to accommodate laws of energy conservation, entropy production, and so forth.

Now, consider two nearby initial conditions, $\boldsymbol{x}_{0,1}$ and $\boldsymbol{x}_{0,2}$, for the point dynamics of Eq. (1), such that:

$$
\boldsymbol{x}_{0,2}-\boldsymbol{x}_{0,1}=\boldsymbol{\epsilon}
$$

with $\boldsymbol{\epsilon}$ a small (vector) perturbation. Eq. (1) will generate two trajectories $\boldsymbol{x}_{1}(t)$ and $\boldsymbol{x}_{2}(t)$. Let us now define the vector $\boldsymbol{v}(t)$ as follows:

$$
\boldsymbol{v}(t):=\lim _{|\boldsymbol{\epsilon}| \rightarrow 0} \frac{\left(\boldsymbol{x}_{2}-\boldsymbol{x}_{1}\right)}{|\boldsymbol{\epsilon}|}
$$

By construction, the vector $v(t)$ belongs to the tangent bundle at $\mathbf{x}_{0,1}$. The vector $\boldsymbol{v}(t)$ is a scaled measure, at time $t$, of the difference between the two trajectories emanating from the two initial conditions. 


\subsection{Derivation of the Vector Dynamics Equation}

The characterization of the dynamical features defined by Eq. (1) can be carried out by viewing the dynamical system as a generalized "advection machine" of all the possible geometric entities that can be defined on the state space [25]. With the term "geometric entities" it is intended the set of measure elements (point, vector, surface, volume, hyper-volumes) that can be constructed in the tangent space $\mathcal{T}_{\boldsymbol{x}_{0}}$ at $\boldsymbol{x}_{0}$.

The algebra of these measure elements (or exterior forms) is called exterior algebra. A comprehensive analysis of exterior algebra can be found in [26]. Use of this formalism allows to introduce a self-consistent system of differential equations for the entries of each measure element.

The simplest measure element is the vector $v$ defined in (3). Its dynamics (intended as the 1-D measure element) can be derived as follows [27].

The point dynamics applied to two initial conditions that satisfy Eq. (2) provides the following ODEs:

$$
\frac{d x_{1}}{d t}=g\left(x_{1}\right), \quad x_{1}(0)=x_{0,1}
$$

and

$$
\frac{d x_{2}}{d t}=\boldsymbol{g}\left(x_{2}\right), \quad \boldsymbol{x}_{2}(0)=\boldsymbol{x}_{0,2} .
$$

Subtracting Eq. (4) from Eq. (5) yields:

$$
\frac{d\left(x_{2}-x_{1}\right)}{d t}=g\left(x_{2}\right)-g\left(x_{1}\right), \quad x_{2}(0)-x_{1}(0)=\epsilon .
$$

Expanding in a Taylor series $\boldsymbol{g}\left(\boldsymbol{x}_{2}\right)$ about $\boldsymbol{x}_{1}$, we obtain

$$
\begin{aligned}
& \frac{d\left(x_{2}-x_{1}\right)}{d t}= \\
& \left\{g\left(x_{1}\right)+\left.\frac{\partial g(x)}{\partial x}\right|_{x_{1}}\left(x_{2}-x_{1}\right)+o\left(\left|x_{2}-x_{1}\right|\right)\right\}-g\left(x_{1}\right), \\
& \boldsymbol{x}_{2}(0)-\boldsymbol{x}_{1}(0)=\boldsymbol{\epsilon}
\end{aligned}
$$

to yield the evolution equation for the difference between the two point dynamics in the tangent space $\mathcal{T}_{\boldsymbol{x}_{1}}$ of $\boldsymbol{x}_{1}$ :

$$
\begin{array}{r}
\frac{d\left(x_{2}-x_{1}\right)}{d t}=J_{g}\left(x_{1}\right) \cdot\left(x_{2}-x_{1}\right)+o\left(\left|x_{2}-x_{1}\right|\right), \\
x_{2}(0)-x_{1}(0)=\epsilon
\end{array}
$$

where

$$
J_{g}\left(x_{1}\right):=\left.\frac{\partial g(x(t))}{\partial x}\right|_{x_{1}}
$$

is the Jacobian matrix of the vector field $\boldsymbol{g}(\boldsymbol{x})$ evaluated at $\boldsymbol{x}_{1}$. It is defined at all times by solving the point dynamics, Eq. (4), for the initial condition in $\boldsymbol{x}_{0,1}$.

To obtain the vector dynamics equation, we first scale Eq. (8) by the norm of the difference between the two initial conditions to obtain

$$
\frac{d}{d t} \frac{\left(x_{2}-x_{1}\right)}{|\epsilon|}=J_{g}\left(x_{1}\right) \cdot \frac{\left(x_{2}-x_{1}\right)}{|\epsilon|}+\frac{o\left(\left|x_{2}-x_{1}\right|\right)}{|\epsilon|} .
$$

If the vector field is smooth (Lipschitz continuous), then the Lipschitz inequality holds for any time $t$ :

$$
\left|x_{0,2}-x_{0,1}\right|=|\boldsymbol{\epsilon}| \Rightarrow\left|x_{2}-x_{1}\right| \leqslant|\epsilon| e^{\mathcal{L} t}
$$

with $\mathcal{L}$ a suitable Lipschitz constant. Subsequently, the second term vanishes under the limit $|\boldsymbol{\epsilon}| \rightarrow 0$, i.e., for any finite time $t$, $o\left(|\boldsymbol{\epsilon}| e^{\mathcal{L} t}\right) /|\boldsymbol{\epsilon}| \rightarrow 0$. Therefore, Eq. (10) provides the sought-after vector dynamics equation:

$$
\frac{d v}{d t}=J_{g}\left(x_{1}\right) \cdot v, \quad v(0)=\mathbf{1},
$$

where $J_{\boldsymbol{g}}\left(\boldsymbol{x}_{1}\right)$ is the Jacobian of the vector field $\boldsymbol{g}$ evaluated along the reference trajectory as defined in (9), and $\mathbf{1}$ is a unit vector at $\boldsymbol{x}_{0}$ taken along any direction.

The vector dynamics of $\boldsymbol{v}$ described by Eq. (12) can be solved only after the integration of Eq. (1) will make available the point dynamics $\boldsymbol{x}_{1}(t)$ required to evaluate $J_{g}\left(\boldsymbol{x}_{1}\right)$. It is emphasized that $\boldsymbol{v} \neq d \boldsymbol{x} / d t$; instead, $\boldsymbol{v}$ provides a measure of the distance between trajectories

To summarize (see for example the comments to Eq. (4) in [28]):

- the dynamics of a small perturbation in $\boldsymbol{x}$ evolves exactly according with Eq. (8), and approximately with the linear dynamic system (12)

- the dynamics of any vector of unit size in the tangent bundle of $\boldsymbol{x}_{0,1}$ evolves exactly according to the linear dynamics (12).

\subsection{Derivation of the Vector Norm Dynamics Equation}

The norm $v$ of the vector $v$ is defined as $v=\sqrt{\boldsymbol{v} \cdot \boldsymbol{v}}$, and its rate of change represents the overall rate of production/consumption of intermediate and product species due to reactions. The equation for the time evolution of $v$ is readily found by taking the scalar product of the left- and right-hand side of Eq. (12) with $\boldsymbol{v}$, and reads

$$
\frac{d v}{d t}=\left(\frac{\boldsymbol{v} \cdot J_{\boldsymbol{g}} \cdot \boldsymbol{v}}{v^{2}}\right) v, \quad v(0)=1
$$

The rate at which $v$ changes (grows/shrinks) with time is governed by the quadratic form enclosed by the parentheses on the right hand side of Eq. (13). It is thus proper to name this coefficient "the (local) rate of stretching of the dynamics," evaluated along the direction identified by the unit vector $\tilde{\boldsymbol{u}}:=\boldsymbol{v} / v$ and defined $^{1}$ as

$$
\omega_{\tilde{\boldsymbol{u}}}:=\tilde{\boldsymbol{u}} \cdot J_{g} \cdot \tilde{\boldsymbol{u}} .
$$

The (local) stretching rate $\omega_{\tilde{u}}$ takes positive/negative values when the dynamics acts so as to stretch/shrink the initial unit vector.

\subsection{Introducing the stretching rates}

In Adrover, et al. [13], it was introduced (i) the tangential stretching rate (TSR) by setting $\tilde{\tau}:=\boldsymbol{g} / \mathrm{g}$, with $\mathrm{g}=|\boldsymbol{g}|$, which spans the vector field direction, and (ii) $N-1$ normal stretching rates spanning the orthogonal complement of the vector field

\footnotetext{
1 To avoid excessive cluttering of the expressions, we have not explicitly replaced a column vector $x$ in an inner product operation of the type $x \cdot y$, with its transpose $x^{T}$ to yield $x^{T} \cdot y$.
} 
subspace. In a two-dimensional system (the simple model of immediate interest), the unit normal vector is obtained as $\tilde{\mathbf{n}}(\boldsymbol{x})=$ $\left\{\tilde{\boldsymbol{\tau}}_{2}(\boldsymbol{x}),-\tilde{\boldsymbol{\tau}}_{1}(\boldsymbol{x})\right\}$. One thus obtains the following definitions of tangential, $\omega_{\tilde{\tau}}$, and normal, $\omega_{\tilde{\mathbf{n}}}$, stretching rates:

$$
\omega_{\tilde{\tau}}:=\tilde{\boldsymbol{\tau}} \cdot J_{g} \cdot \tilde{\boldsymbol{\tau}} \quad \omega_{\tilde{\mathbf{n}}}:=\tilde{\mathbf{n}} \cdot J_{g} \cdot \tilde{\mathbf{n}} .
$$

For $\omega_{\tilde{\tau}}\left(\omega_{\tilde{\mathbf{n}}}\right)>0$, the tangential (normal) perturbations are amplified, while for $\omega_{\tilde{\tau}}\left(\omega_{\tilde{\mathbf{n}}}\right)<0$ they are damped.

However, this definition based on the local Jacobian degenerates, as discussed in the next section, when the trajectory crosses a region of complex eigenvectors.

To overcome this shortcoming, we will combine the TSR definition with the eigen-decomposition of the Jacobian matrix of the vector field as discussed in the following section.

\subsection{Stretching rates and eigenvalues}

The Jacobian of the vector field can be always decomposed as $J_{\boldsymbol{g}}=A \cdot \Lambda \cdot B$, where $A=\left\{\boldsymbol{a}_{j}\right\}_{j=1, N}$ and $B=\left\{\boldsymbol{b}^{i}\right\}_{i=1, N}$ are the right and left normalized eigenvector matrices of $J_{g}$, respectively, and $\Lambda=\left\{\lambda_{j}^{i}\right\}_{i, j=1, N}$ is the eigenvalue matrix of $J_{\boldsymbol{g}}$.

Evaluating the stretching rates along the eigendirections, $\boldsymbol{a}_{j}$, yields:

$$
\omega_{\boldsymbol{a}_{j}}:=\boldsymbol{a}_{j} \cdot J_{\boldsymbol{g}} \cdot \boldsymbol{a}_{j}
$$

By definition of eigendirection, we have that $J_{\boldsymbol{g}} \cdot \boldsymbol{a}_{j}=\lambda_{j} \boldsymbol{a}_{j}$, so that $\omega_{a_{j}}$ becomes

$$
\omega_{\boldsymbol{a}_{j}}=\boldsymbol{a}_{j} \cdot J_{\boldsymbol{g}} \cdot \boldsymbol{a}_{j}=\boldsymbol{a}_{j} \cdot \lambda_{j} \boldsymbol{a}_{j}=\left|\boldsymbol{a}_{j}\right|^{2} \lambda_{j}=\lambda_{j} .
$$

since $\left|\boldsymbol{a}_{j}\right|=1$ by construction. Thus, the stretching rate $\omega_{\boldsymbol{a}_{j}}$ evaluated along $\boldsymbol{a}_{j}$ coincides with the eigenvalue $\lambda_{j}$ corresponding to that eigendirection.

The unit vector $\tilde{\tau}$ can be rewritten after projecting the vector field over the right eigenvector basis as

$$
\tilde{\boldsymbol{\tau}}=\frac{\boldsymbol{g}}{\mathrm{g}}=\frac{1}{\mathrm{~g}} \sum_{i=1}^{N} \boldsymbol{a}_{i} f^{i} \text {, with } f^{i}:=\boldsymbol{b}^{i} \cdot \boldsymbol{g}, \text { and } \boldsymbol{g}=\sum_{i=1}^{N} \boldsymbol{a}_{i} f^{i}
$$

Given that $J_{g}=A \cdot \Lambda \cdot B$, we now have

$$
\begin{aligned}
\omega_{\tilde{\boldsymbol{\tau}}} & =\tilde{\boldsymbol{\tau}} \cdot J_{\boldsymbol{g}} \cdot \tilde{\boldsymbol{\tau}}=\frac{1}{\mathrm{~g}^{2}}(\boldsymbol{g} \cdot A \cdot \Lambda \cdot B \cdot \boldsymbol{g})=\frac{\boldsymbol{g}}{\mathrm{g}^{2}} \sum_{i=1}^{N} \boldsymbol{a}_{i} \lambda_{i}\left(\boldsymbol{b}^{i} \cdot \boldsymbol{g}\right) \\
& =\frac{\boldsymbol{g}}{\mathrm{g}^{2}} \sum_{i=1}^{N} \boldsymbol{a}_{i} \lambda_{i} f^{i}=\frac{1}{\mathrm{~g}^{2}} \sum_{i=1}^{N}\left(\boldsymbol{g} \cdot \boldsymbol{a}_{i}\right) \lambda_{i} f^{i}
\end{aligned}
$$

Expanding $\boldsymbol{g}$ in terms of eigen-modes, one obtains

$$
\boldsymbol{g} \cdot \boldsymbol{a}_{i}=\left(\sum_{k=1}^{N} \boldsymbol{a}_{k} f^{k}\right) \cdot \boldsymbol{a}_{i}=\sum_{k=1}^{N} f^{k}\left(\boldsymbol{a}_{k} \cdot \boldsymbol{a}_{i}\right),
$$

where $\boldsymbol{a}_{k} \cdot \boldsymbol{a}_{i}$ is the direction cosine (the phase) between $\boldsymbol{a}_{i}$ and $\boldsymbol{a}_{k}$ (with $\left|\boldsymbol{a}_{k} \cdot \boldsymbol{a}_{i}\right| \leq 1$ ).
With this result, Eq. (18) becomes

$$
\begin{aligned}
\omega_{\tilde{\tau}} & =\sum_{i=1}^{N}\left(\frac{1}{\mathrm{~g}^{2}} \sum_{k=1}^{N} f^{k}\left(\boldsymbol{a}_{k} \cdot \boldsymbol{a}_{i}\right)\right) \lambda_{i} f^{i} \\
& =\sum_{i=1}^{N}\left(\frac{f^{i}}{\mathrm{~g}^{2}} \sum_{k=1}^{N} f^{k}\left(\boldsymbol{a}_{k} \cdot \boldsymbol{a}_{i}\right)\right) \lambda_{i}=\sum_{i=1}^{N} W_{i} \lambda_{i}
\end{aligned}
$$

with

$$
W_{i}:=\frac{f^{i}}{\mathrm{~g}} \frac{\boldsymbol{g} \cdot \boldsymbol{a}_{i}}{\mathrm{~g}}=\frac{f^{i}}{\mathrm{~g}} \sum_{k=1}^{N} \frac{f^{k}}{\mathrm{~g}}\left(\boldsymbol{a}_{k} \cdot \boldsymbol{a}_{i}\right) .
$$

The vector field is an invariant direction for the (locally linearized) dynamics as well as the eigendirections. The unit vector $\tilde{\boldsymbol{\tau}}$ tangent to the vector field $\boldsymbol{g}$ changes according with the rate $\omega_{\tilde{\tau}}=\sum_{i=1}^{N} W_{i} \lambda_{i}$ because of the action of the (linearized) dynamics as represented by $J_{g}$. By construction, this term takes the maximum value when all $\boldsymbol{a}_{i}$ are co-linear with $\boldsymbol{g}$, that is:

$$
\frac{\boldsymbol{g} \cdot \boldsymbol{a}_{i}}{\mathrm{~g}}=\sum_{k=1}^{N} \frac{f^{k}}{\mathrm{~g}}\left(\boldsymbol{a}_{k} \cdot \boldsymbol{a}_{i}\right) \leq \frac{f^{i}}{\mathrm{~g}}
$$

and substituting this result in Eqs. (19)-(21), provides the upper bound

$$
\omega_{\tilde{\tau}} \leq \sum_{i=1}^{N}\left(\frac{f^{i}}{\mathrm{~g}}\right)^{2} \lambda_{i}
$$

This shows that, because of the quadratic term, the sign of $\omega_{\tilde{\tau}}$ depends on those of the prevailing eigenvalues.

Remark 1. To summarize, the stretching rate along an eigendirection simply coincides with the corresponding eigenvalue. Instead, the (tangential) stretching rate along the vector field, which is an invariant direction of the dynamics, is a weighted average of all eigenvalues $\lambda_{i}$, with weights $W_{i}$ that according to Eq. (21) depend on:

- the normalized amplitude $f^{i}$ of the $i$-th mode,

- the degree of co-linearity of the eigenvector $\boldsymbol{a}_{i}$ with respect to the vector field $g$.

\subsection{Tangent Space Decomposition induced by TSR}

After ordering the terms in the sum which defines $\omega_{\tilde{\tau}}$ by the descending ${ }^{2}$ value of the modulus of the eigenvalue, and setting for simplicity $\boldsymbol{g} \cdot \boldsymbol{a}_{i}=f^{i}$ for all modes, we have

$$
\omega_{\tilde{\tau}}=\sum_{r=1}^{L}\left(\frac{f^{r}}{\mathrm{~g}}\right)^{2} \lambda_{r}+\sum_{a=L+1}^{K-1}\left(\frac{f^{a}}{\mathrm{~g}}\right)^{2} \lambda_{a}+\sum_{s=K}^{N}\left(\frac{f^{s}}{\mathrm{~g}}\right)^{2} \lambda_{s},
$$

\footnotetext{
2 The ordering of the eigenvalues in the G-Scheme and CSP is different ( $i=1$ is the slowest (smallest) in the G-Scheme whereas is the fastest (largest) in CSP. In this paper, we adopted the CSP ordering.
} 
where the labels " $r$ ", " $a$ ", " $s$ " denote fast, active, slow modes, respectively. Let us now consider a typical situation for which, at some point in the phase space, there exists a number of fast and slow modes with a vanishing amplitude, that is $\left\{f^{r}=\boldsymbol{b}^{r} \cdot \boldsymbol{g} \approx 0\right\}_{r=1, \ldots, L}$ and $\left\{f^{s}=\boldsymbol{b}^{s} \cdot \boldsymbol{g} \approx 0\right\}_{s=K, \ldots, N}$, with $L<$ $K$.

This situation can develop when there exist two spectral gaps between the slow and active subspaces, $\varepsilon_{S}=\left|\lambda_{L} / \lambda_{L+1}\right|$, and between the active and slow subspaces, $\varepsilon_{r}=\left|\lambda_{K-1} / \lambda_{K}\right|$, both (much) smaller than one.

In such a case, it happens that

$$
\omega_{\tilde{\tau}} \approx \sum_{a=L+1}^{K-1}\left(\frac{f^{a}}{\mathrm{~g}}\right)^{2} \lambda_{a}
$$

which implies that only the active scales contribute to $\omega_{\tilde{\tau}}$. If an eigenvalue is positive, say $\lambda_{a+}$, it is likely that $f^{a+}$ will be the largest of all active mode amplitudes. In this case $\omega_{\tilde{\tau}}$ will be mostly affected by $\lambda_{a+}$.

From Eq. (23), three scales can be identified: (i) one associated with $\omega_{\tilde{\tau}}$, which is the most energy containing scale; (ii) two others associated with $\lambda_{K-1}$ and $\lambda_{L+1}$, which are the fastest and slowest scales, respectively, contributing to $\omega_{\tilde{\tau}}$, and these bracket the range of active (energy containing) scales. The integers $K$ and $L$ underline a decomposition of the tangent space $\mathcal{T}_{x}$ in three subspaces: the fast subspace $\mathcal{F}_{T S R}(r=1, \ldots, L)$, the active $\mathcal{A}_{T S R}(a=L+1, \ldots, K-1)$, and the slow $\mathcal{S}_{T S R}$ $(s=K, \ldots, N)$, such that $\mathcal{T}_{\boldsymbol{x}}=\mathcal{F}_{T S R} \bigoplus \mathcal{A}_{T S R} \bigoplus \mathcal{S}_{T S R}$. This three-fold decomposition is analogous to that employed in the G-Scheme framework proposed in [23]. In the CSP method, the contribution of the $M$ fastest modes $(r=1, \ldots, M)$ to the system dynamics is considered negligible until the following inequality is satisfied

$$
\tau_{M+1} \sum_{r=1}^{M} a_{r}^{j} f^{r}<\epsilon^{j}=\operatorname{rtol}^{j}\left|x^{j}\right|+\operatorname{atol}^{j}, \quad j=1, \ldots, N
$$

where $\tau_{M+1}=1 /\left|\lambda_{M+1}\right|$, and $\epsilon=\left\{\epsilon^{j}\right\}_{j=1, N}$ is a user-defined error vector. In all the calculation carried out in this work, we set $\mathrm{rtol}^{j}=1.0 \times 10^{-2}$, and atol ${ }^{j}=1.0 \times 10^{-8}$, for all $j$. In CSP, the first integer $M$ for which the inequality is not satisfied for all $N$ components of the state $\boldsymbol{x}$, identifies the decomposition of the tangent space as $\mathcal{T}_{\boldsymbol{x}}=\mathcal{F}_{C S P} \bigoplus \mathcal{S}_{C S P}$, with $\mathcal{F}_{C S P}=\left\{\boldsymbol{a}_{r}\right\}_{r=1, \ldots, M}$ atnd $\mathcal{S}_{C S P}=\left\{\boldsymbol{a}_{s}\right\}_{s=M+1, \ldots, N}$.

Note that the "slow" modes in the CSP nomenclature correspond to the union of the "slow" and "active" modes in the G-Scheme nomenclature, that is $\mathcal{A}_{T S R} \bigoplus \mathcal{S}_{T S R}=\mathcal{S}_{C S P}$, while the fast subspaces coincide: $\mathcal{F}_{T S R}=\mathcal{F}_{C S P}$.

\subsection{CSP and TSR Analyses}

At this stage we can establish a bridge connecting the CSP concepts and the TSR analysis, by observing that $\lambda_{M+1}$ in CSP and $\lambda_{L+1}$ in TSR are the same scale, that is, the fastest of the slow/active scales. However, the TSR analysis identifies two other significant scales: $\lambda_{K-1}$, the slowest of the active scales, and $\omega_{\tilde{\tau}}$ itself, which is the scale containing most of the energy.
Equations (19)-(21) provide a method to compute the TSR on the basis of the local eigensystem, but the inability of handling the presence of complex eigenvalues and eigenvectors is still unsolved. After a discussion of some preliminary results, we will propose a simple modification to circumvent this difficulty.

\section{The Branched-Chain Reactions Isothermal Williams Model}

In this section, we consider the Williams' model as a prototype of the kinetics of a real chemical mechanism. The Williams' model for isothermal branched-chain reactions [20], involves three irreversible reactions: $\mathrm{R} \rightarrow \mathrm{C}$ (initiation), $\mathrm{R}+\mathrm{C} \rightarrow \alpha \mathrm{C}+\mathrm{P}$ (propagation), $\mathrm{C} \rightarrow \mathrm{P}$ (termination), where $\mathrm{R}=$ reactants, $\mathrm{C}=$ intermediates, $\mathrm{P}=$ products, and the three reaction rates $k_{i, p, t}$ are taken constant due to the assumed isothermicity. The branching factor $\alpha$ takes values $\alpha>1$ to mimic branching propagation. A suitable non dimensionalization of Williams' model has been proposed in [21]. The three non dimensional ODEs that describe the kinetics of the model (1) are

$$
\begin{aligned}
\frac{d x_{1}}{d \tau} & =-x_{1}-x_{1} x_{2}, \\
\epsilon \frac{d x_{2}}{d \tau} & =x_{1}+(\alpha-1) x_{1} x_{2}-\gamma x_{2}, \\
\gamma \frac{d x_{3}}{d \tau} & =\gamma x_{2}+x_{1} x_{2},
\end{aligned}
$$

with initial conditions $\left(\mathrm{x}_{1}(0), \mathrm{x}_{2}(0), \mathrm{x}_{3}(0)\right)=(1,0,0)$. The non dimensional time is given by $\tau:=t k_{i}$, with $t$ being the dimensional time. The non dimensional state variables are $x_{1}:=r / r_{0}$, $x_{2}:=c k_{p} / k_{i}$, and $x_{3}:=p k_{p} / k_{t}$, with $r, c$, and $p$ the molar concentrations of $\mathrm{R}, \mathrm{C}$, and $\mathrm{P}$, respectively, and $r_{0}$ the initial molar concentration of $\mathrm{R}$. Because the third equation is decoupled from the other two, Williams' model is effectively twodimensional, with state vector defined by the pair $\left(x_{1}, x_{2}\right)$. The dimensionless rate constants are defined as $\epsilon=k_{i} /\left(k_{p} r_{0}\right)$ and $\gamma=k_{t} /\left(k_{p} r_{0}\right)$. A super-critical trajectory for $\alpha=2, \epsilon=0.01, \gamma$ $=0.5 \gamma_{c r}$, with $\gamma_{c r}=\alpha-1$, is plotted in the phase space $\left(x_{1}, x_{2}\right)$ in Fig. 1 (solid black curve).

The trajectory is nearly tangent to the fast eigenvector (dark gray arrow) $\boldsymbol{a}_{1}$ at $(1,0)$, and to the slow eigenvector (light gray arrow) $\boldsymbol{a}_{2}$ at $(0,0)$, and, at time $t \approx 0.3\left(x_{1} \approx 0.15\right.$ in Fig. 1$)$, it lands on the QSSA of the Slow Invariant Manifold (dashed gray line) when both mode amplitudes have the same magnitude, and eventually reaches the equilibrium point $(0,0)$.

The region of complex eigenvalues lies above the dot dashed line in Fig. 1. The dotted line is the locus of zero real part of the complex conjugate eigenvalues.

The eigenvalue analysis carried out along the supercritical trajectory exhibits the evolution shown in Fig. 2. There exists a first stage during which a pair of eigenvalues with positive real parts form; this stage ends when the pair of positive eigenvalues merge to form a complex conjugate pair with positive real parts. Eventually, the complex conjugate pair crosses the imaginary axis so that the real parts becomes negative. Finally, the imaginary parts reduce to zero, and a pair of real negative eigenval- 


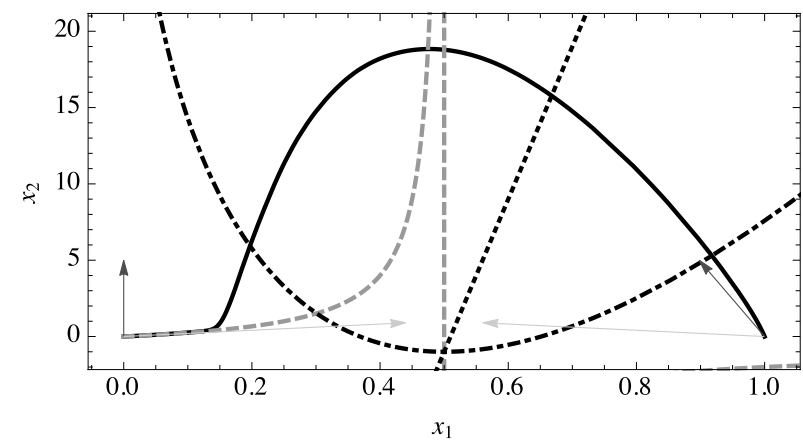

Figure 1: Trajectory (solid line), complex conjugate eigenvalues region boundary (dot dashed line), zero real part of eigenvalues (dotted line), QSSA of SIM (dashed gray line), and fast/slow eigenvector (dark/light gray arrows) at $(1,0)$ and $(0,0)$.

ues emerge and remain until the system reaches the equilibrium state.

Note that when the real parts of the eigenvalues cross zero, the eigenvalues' modulus is defined by the imaginary part and is non-zero (see Fig. 2). Note for future consideration that introducing a time scale as the reciprocal of the sole real part leads to an infinite scale at the zero crossing, while the reciprocal of the modulus will always remain bounded.

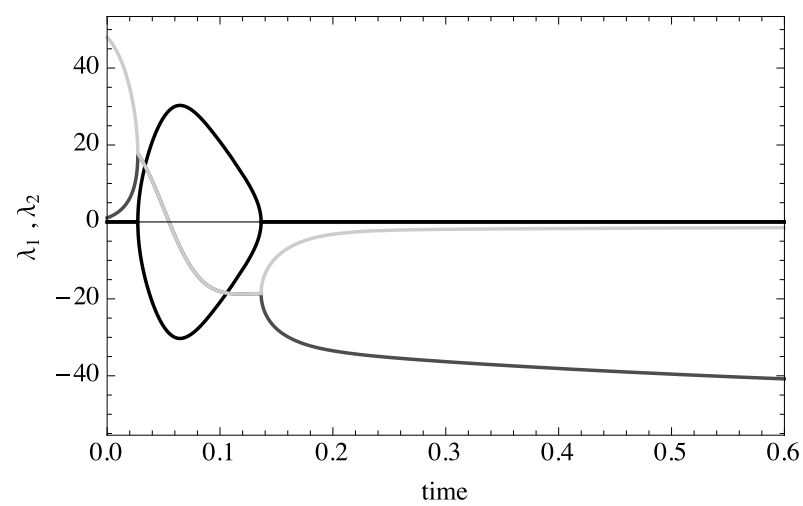

Figure 2: Real part of eigenvalues ( $\lambda_{1}$ (dark gray line), $\lambda_{2}$ (light gray line)); imaginary part of eigenvalues (black solid line); $\alpha=2, \epsilon=0.01, \gamma=0.5 \gamma_{c r}$, $\gamma_{c r}=\alpha-1$.

In the first stage of the ignition, whose detail is shown in Fig. 3(a), when the eigenvalues are both positive and real, the slow eigenvector (light gray arrow), while approaching the complex region, rotates toward the direction of the fast eigenvector (dark gray arrow) which is nearly tangent to the trajectory, until the fast and slow eigenvectors become co-linear at the complex region boundary. In the third stage of the ignition, whose detail is shown in Fig. 3(b), when the eigenvalues are both negative and real, the slow eigenvector (light gray arrow) at the complex region boundary is co-linear with the fast eigenvector (dark gray arrow) and also co-linear with the vector field. While leaving the complex region, it rotates away from the direction of the fast eigenvector (dark gray arrow), until the slow eigenvector (light gray arrow) becomes nearly tangent to the trajectory in the slow approach to the fixed point.

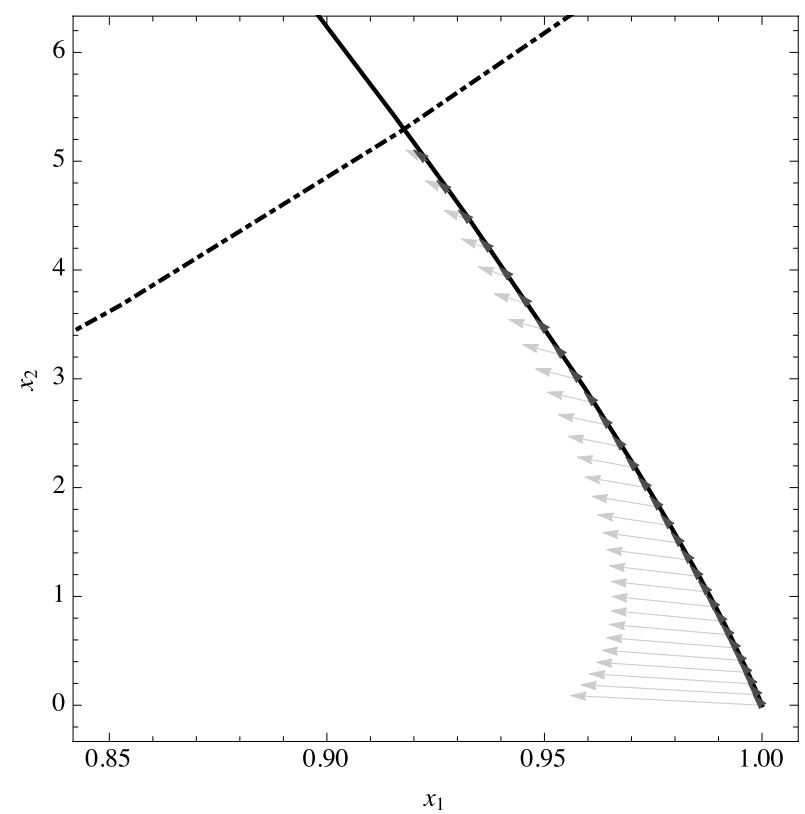

(a)

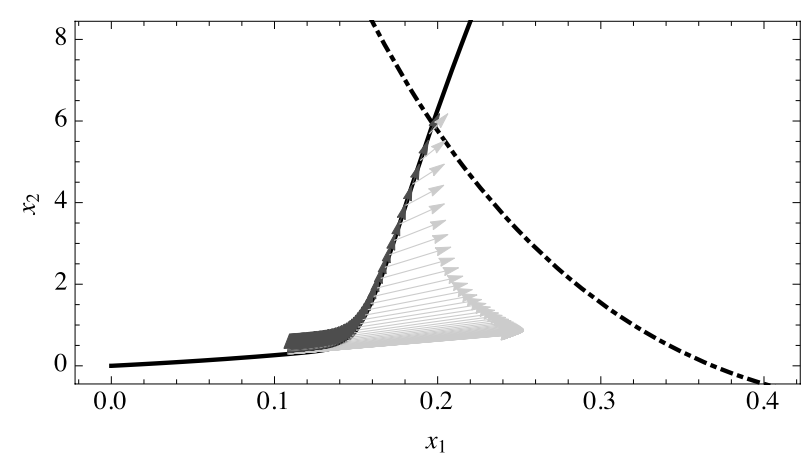

(b)

Figure 3: Eigenvectors evolution during (a) the first stage, and (b) the third stage of ignition along the trajectory (fast eigenvector $a_{1}$ (dark gray arrow), slow eigenvector $a_{2}$ (light gray arrow))

Within the complex region, the eigenvectors and eigenvalues are complex conjugates, no time scale separation exists and the direction along which the single time scale is acting is simply the local tangent of the trajectory, that is the vector field itself. This conjecture can be drawn by a continuity argument noting that the two real eigenvectors both enter and leave the complex region being co-linear among themselves and with the vector field (see Fig. 3).

One way to elucidate the role of complex eigenvalues and eigenvectors, is the following. Consider the state point $\bar{x}=$ $\boldsymbol{x}(\bar{t})$ of the trajectory that at time $\bar{t}$ is found within the complex region. Next, integrate the dynamics of the small perturbations $\delta x$ about $\bar{x}$ for large times, on the basis of the linear system

$$
\frac{d(\delta \boldsymbol{x})}{d \tau}=\boldsymbol{g}(\overline{\boldsymbol{x}})+J_{g}(\overline{\boldsymbol{x}}) \delta \boldsymbol{x}, \quad \delta \boldsymbol{x}(\tau=0)=0
$$

from which one can construct the state vector as $\boldsymbol{x}(\bar{t}+\tau)=$ 
$\overline{\boldsymbol{x}}(\bar{t})+\delta \boldsymbol{x}(\tau)$. By construction, the eigenvectors and eigenvalues of $J_{g}(\bar{x})$ are complex conjugates. The trajectory obtained by selecting $\overline{\boldsymbol{x}}$ in the regions of eigenvalues with positive/negative real part (specifically: one equal to $\overline{\boldsymbol{x}}\left(t_{0}=0.04\right)=$ $\{0.822737,9.97036\}$, at which the eigenvalues are $\left\{\lambda_{1}, \lambda_{2}\right\}=$ $\{10.6517-20.8581 i, 10.6517+20.8581 i\}$, and the next equal to $\overline{\boldsymbol{x}}\left(t_{0}=0.105\right)=\{0.270419,12.9093\}$, at which the eigenvalues are $\left\{\lambda_{1}, \lambda_{2}\right\}=\{-18.4337-18.859 i,-18.4337+18.859 i\}$ ), are shown in Figs. 4 and 5, respectively. These results clearly illustrate that the trajectories of the linearized system are outward/inward spirals, the outward spiral tending to infinity, and the inward spiral approaching the fixed point of the linearized system. By analogy with the motion of a point-mass, one can interpret the real part of the complex eigenvalue as affecting the tangential acceleration at one point of the spiral motion, and the imaginary part of the complex eigenvalue as affecting the centripetal acceleration responsible of the deviation of the trajectory from a straight line.

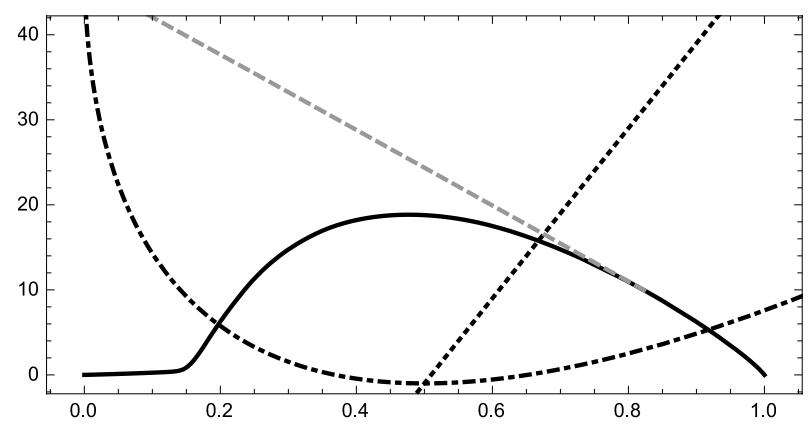

(a)

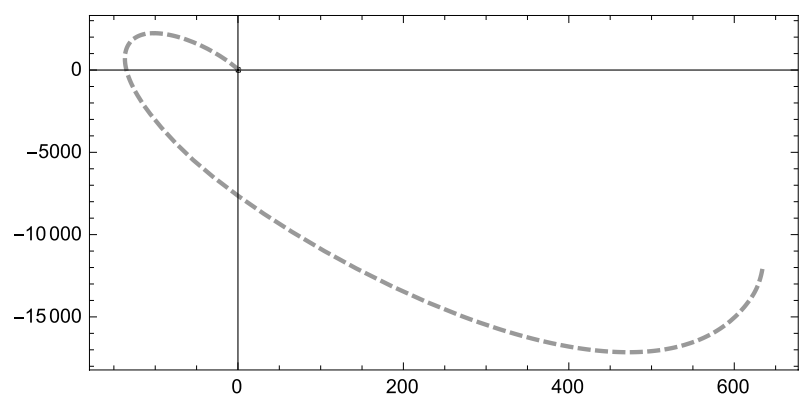

(b)

Figure 4: Dynamics of linearized system at large times: an initial point chosen in the region of eigenvalues with positive real part generates an outward spiral tending to infinity. Trajectory of the full system (black solid line), trajectory of the linearized system (dashed gray line), complex conjugate eigenvalues region boundary (dot dashed line), zero real part of eigenvalues (dotted line).

To summarize, we can say that at one point with two real eigenvalues and eigenvectors, it is possible to project the vector field onto the slow and fast eigendirections. This is not possible when the eigenvalues are complex, but the acceleration vector, $\ddot{\boldsymbol{x}}=J_{\boldsymbol{g}} \cdot \boldsymbol{g}$, can still be decomposed into tangential and normal components: the real part relates to the rate of growth of tangential perturbations, and the imaginary part to the rate of change of the direction of the vector field.

It is instructive to provide a visual representation of the action

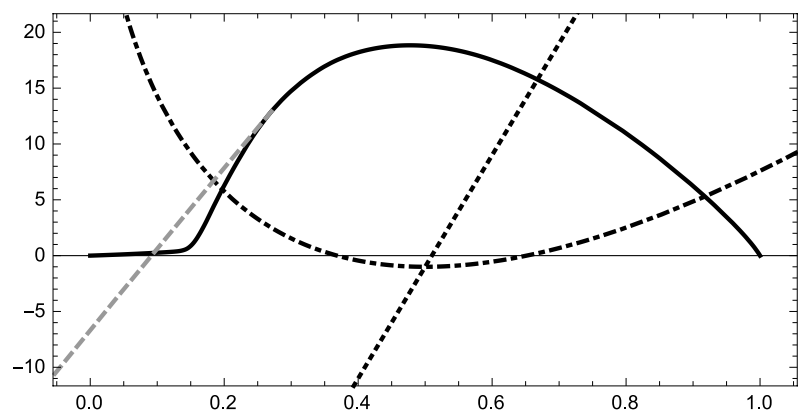

(a)

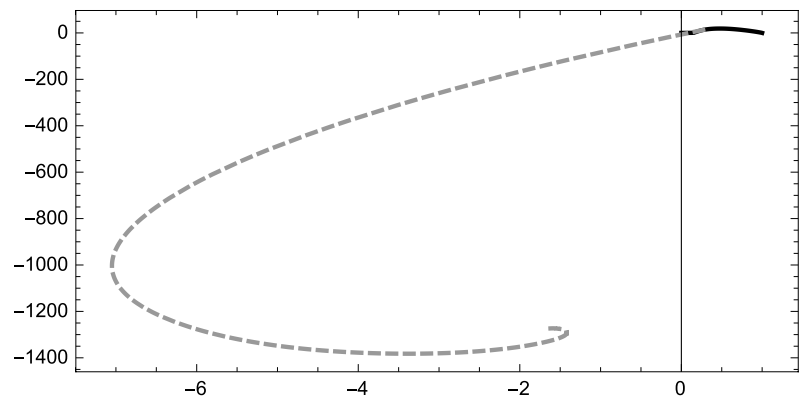

(b)

Figure 5: Dynamics of linearized system at large times: an initial point chosen in the region of eigenvalues with negative real part generates an inward spiral approaching the fixed point of the linearized system. Trajectory of the full system (black solid line), trajectory of the linearized system (dashed gray line), complex conjugate eigenvalues region boundary (dot dashed line), zero real part of eigenvalues (dotted line).

of the vector dynamics, Eq. (12), when applied to the Williams model. With this aim, we portrait in Fig. 6 the evolution of a small circle drawn about the initial condition and advected in time by the dynamical system (1). The small circle can be obtained by rotating $360^{\circ}$ the unit vector 1 , defining the initial condition of the vector dynamics (12), which hinges at the initial condition of the trajectory. As time progresses, the union of unit vectors $\mathbf{1}$ forming the initial circle at time zero is advected by the vector dynamic equation (12), thus mapping the initial circle at time zero into some other closed line at all subsequent times.

In Fig. 6, the explosive nature associated with the occurrence of the two positive eigenvalues translates in the enlargement of the initially circular "probe" (note that the axis have independent ranges) both along the tangential and the other (different from the tangential) directions. After having reached its maximum size, the closed line starts to experience the dissipative (contractive) nature of the two negative eigenvalues until its size shrinks first to a one dimensional object moving along the SIM, and eventually collapses to a zero-dimensional object at the fixed point.

Note that the singular value decomposition of the Jacobian matrix provides both the directions of extremal dilatation (growth/shrinkage) of the circular probe and their extent (singular values). The ratio of the largest to the smallest singular value provides an estimate of the condition number of the Ja- 
cobian matrix. A large condition number is an indication of ill-conditioning of the Jacobian matrix.

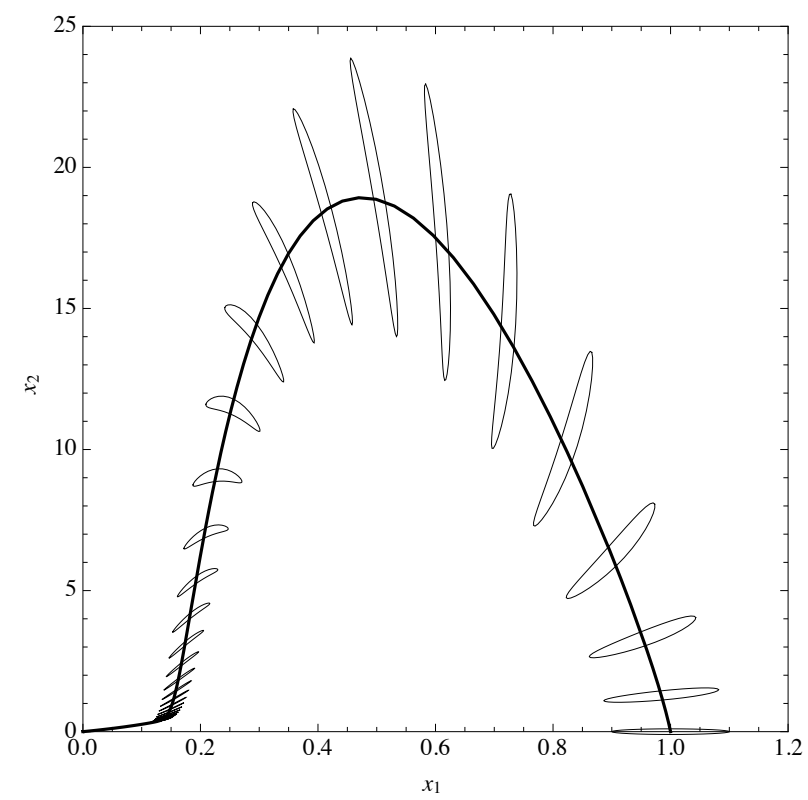

Figure 6: Evolution of a small circle drawn about the initial condition and advected in time by the dynamical system (axis have different scales).

\subsection{CSP Analysis}

The CSP criterion, Eq. (25), used to partition the tangent space in fast and slow subspaces requires computing the mode amplitudes as well as the eigenvalues already shown in Fig. 2. A log-linear plot of the mode amplitudes corresponding to Williams' model is shown in Fig. 7. For easy reference, we will refer to the time period when the eigenvalues are real and positive/negative as Period 1 (P1) and Period 3 (P3), respectively, while when the eigenvalues are complex as Period $2(\mathrm{P} 2)$.

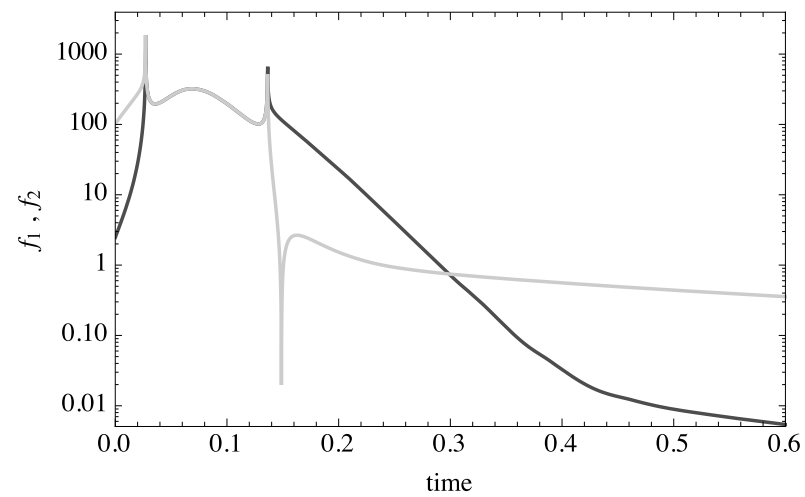

Figure 7: Absolute value of CSP mode amplitudes $\left(f_{1}=\left|\boldsymbol{b}^{1} \cdot \boldsymbol{g}\right|\right.$ (dark gray line), $f_{2}=\left|\boldsymbol{b}^{2} \cdot \boldsymbol{g}\right|($ light gray line $\left.)\right) ; \alpha=2, \epsilon=0.01, \gamma=0.5 \gamma_{c r}, \gamma_{c r}=\alpha-1$.

In Williams' model, it is possible to obtain the analytical expressions for the two eigenvalues and eigenvectors. We can thus unambiguously refer to each mode as Mode 1 and 2. Mode 1 (dark gray line) is slow (smallest positive eigenvalue) in P1, and fast (largest negative eigenvalue) in the early stage of P3, while Mode 2 (light gray line) has the opposite behavior, first fast then slow. In P1, Mode 2 has the largest amplitude and the largest positive eigenvalue. The application of the CSP criterion returns $M=0$, that is the fast subspace is empty, and the active (fast) scale is associated with $\lambda_{2}>0$ (light gray line). In P2, there is no scale separation and thus no fast/slow decoupling is possible. In P3, Mode 1 has the largest amplitude up to time $t \approx 0.3$ and the largest negative eigenvalue. Thus, even during this phase (P3a), we obtain $M=0$, the fast subspace is empty, and the active (fast) scale is associated with $\lambda_{1}<0$ (dark gray line). At $t \approx 0.3$, both modes have the same amplitude, $f_{1}=f_{2}$, and thus are equally important. For $t \gtrsim 0.3, f_{1}$ becomes smaller than $f_{2}$, and thus, Mode 1 becomes exhausted, the fast subspace is spanned by Mode 1, and the active (slow) scale is associated with $\lambda_{2}<0$ (light gray line). It is interesting to note that, although both eigenvalues are negative and real after time $t \approx 0.138$ as shown in Fig. 2, the trajectory remains nearly tangent to the fast eigenvector until $t \approx 0.345$, when the SIM is reached: there the trajectory becomes tangent to the slow eigenvector (light gray line). This behavior is monitored by inspection of the fast mode amplitude (dark gray line) which becomes smaller than the slow mode amplitude (light gray line) only after $t \approx 0.32$ (when they are alike; see Fig. 7).

Remark 2. This confirms that the negative sign of the eigenvalues and the existence of a gap between the two are only necessary conditions for the development of a SIM, the sufficient condition requires the vanishing of the fast mode amplitude.

Note that the transition from $M=0$ to $M=1$ is abrupt as soon as the error threshold in the criterion of Eq. (25) allows one to declare that $f_{1}$ is negligible. When this happens the active scale abruptly switches from $\lambda_{1}$ to $\lambda_{2}$. On the basis of the mode classification described here below, the CSP analysis can be continued to establish cause-effect relationships by resorting to the different definitions of indices that estimate the relative contribution of any reaction to the making of the amplitude of a mode (Mode Participation Index), of a time scale (Time Scale Participation Index), or of the time rate of change of a species (Slow and Fast Importance Index). This route of investigation has been fully pursued by Goussis and co-workers in [3].

\subsection{TSR Analysis}

Figure 8 compares the evolution of the tangential (dashed black line)/normal (dot-dashed black line) stretching rates, computed using the definitions in Eq. (15), with respect to variations of the eigenvalues. We observe that $\omega_{\tilde{\tau}}$ follows the fast eigenvalue (positive (light gray line) in P1 and negative (dark gray line) in P3) up to time $t \approx 0.2$. In the interval $t \approx 0.2-0.45$, the two stretching rates switch between the eigenvalues, so that after $t \approx 0.45, \omega_{\tilde{\tau}}$ follows the slow eigenvalue (light gray line). The opposite trend is followed by the normal rate.

Remark 3. However, both stretching rates take very large and nearly opposite values in $P 2$. This is because $J_{g}$ cannot decou- 


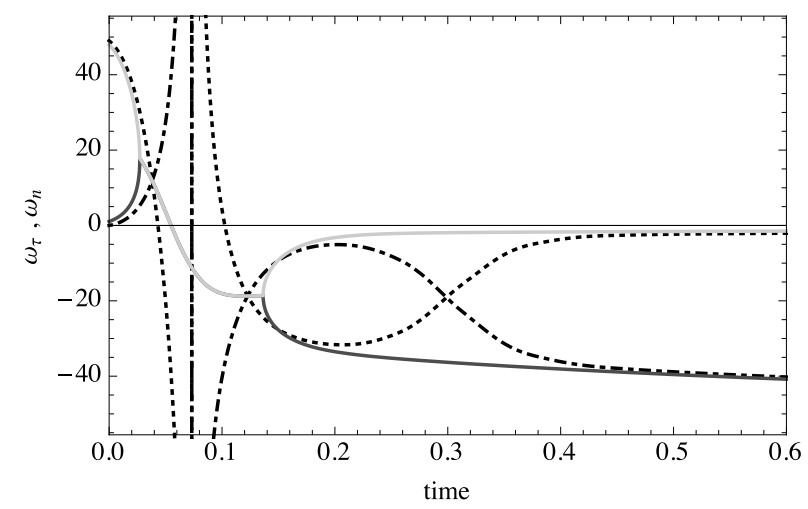

Figure 8: Stretching rates evaluated with Eq. (15); $\omega_{\tilde{\tau}}$ (dashed black line), $\omega_{\tilde{\boldsymbol{n}}}$ (dot-dashed black line), $\operatorname{Re}\left(\lambda_{1}\right)$ (light gray line), $\operatorname{Re}\left(\lambda_{2}\right)$ (dark gray line).

ple the dynamics in tangential and normal components when the eigenvalues and eigenvectors are complex.

When this occurs, instead of resorting to the dichotomies fast/slow, tangential/normal, one could resort to that of compression/expansion of the infinitesimal volume in phase plane and consider the volumetric stretching rate, $\omega_{V}$, given by the rate of change of a differential volume $d V$. It can be shown that in 2D the following relations hold:

$$
\omega_{V}:=\operatorname{tr}(D)=\lambda_{1}+\lambda_{2}=\omega_{\tilde{\tau}}+\omega_{\tilde{n}} .
$$

where $D=\left(J_{g}+J_{g}^{T}\right) / 2$ is the symmetric part of $J_{g}$.

One can therefore choose to set $\tilde{\omega}_{\tilde{\tau}}=\tilde{\omega}_{\tilde{n}}=\omega_{V} / 2$ when $\lambda_{1,2}$ are complex conjugates. This modification of the original definition of tangential and normal stretching rates produces the result shown in Fig. 9, where obviously the degeneracy problem is circumvented.

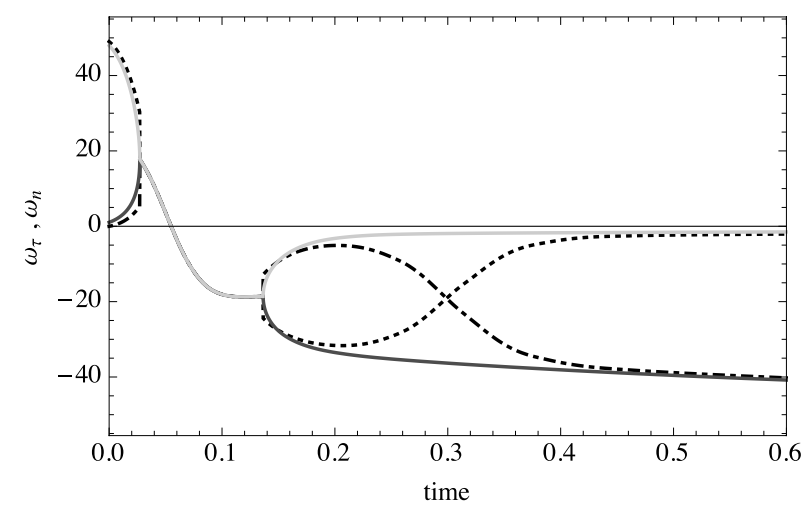

Figure 9: Stretching rates evaluated with Eq. (28) $\tilde{\omega}_{\tilde{\tau}}$ (dashed black line), $\tilde{\omega}_{\tilde{\boldsymbol{n}}}$ (dot-dashed black line), $\operatorname{Re}\left(\lambda_{1}\right)$ (light gray line), $\operatorname{Re}\left(\lambda_{2}\right)$ (dark gray line).

A positive/negative determinant of the Jacobian is the local measure of the rate of growth/decay, respectively, of an elementary volume while being advected by the flow dynamics. Note that the determinant of the Jacobian is never zero along the trajectory as shown in Fig. 10 and so the Jacobian matrix $J_{g}$ is never singular.

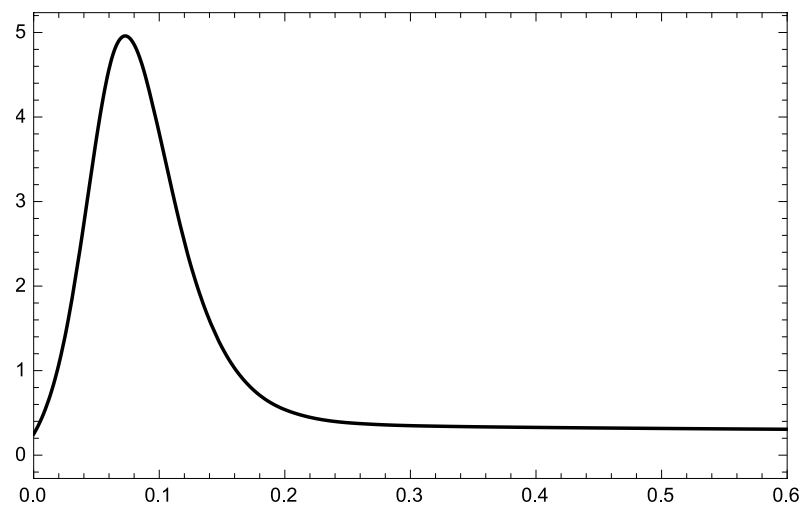

Figure 10: Determinant of the Jacobian $J_{g}$ along the trajectory.

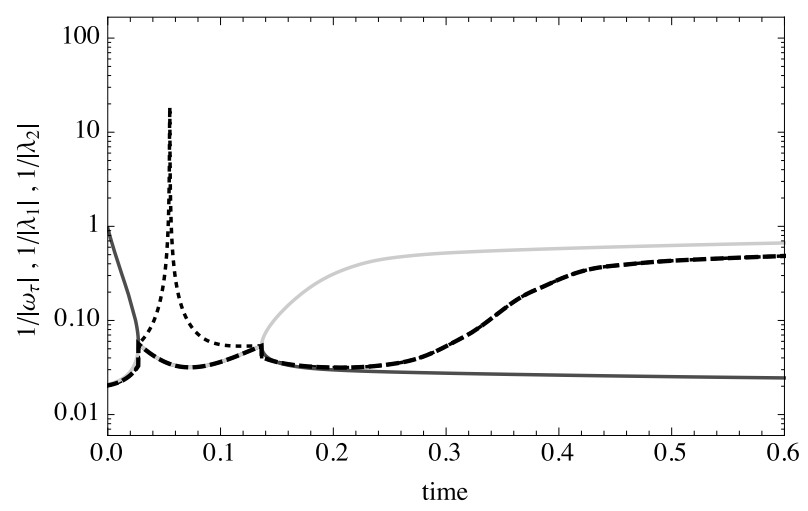

Figure 11: $1 /\left|\lambda_{1,2}\right|$ (light/dark gray line), $1 / \tilde{\omega}_{\tilde{\tau}}$ (dashed gray line); $1 / \hat{\omega}_{\tilde{\tau}}$ (dashed black line); the reciprocal of $\left|\tilde{\omega}_{\tilde{\tau}}\right|$ goes to infinity when $\tilde{\omega}_{\tilde{\tau}}$ crosses the imaginary axis in Fig. 9, whereas $\hat{\omega}_{\tilde{\tau}}$ defined as in Eq. (29) is well defined.

Time scales estimated as the reciprocals of $\tilde{\omega}_{\tilde{\tau}}=\omega_{V} / 2$, and of the two eigenvalues are shown in Fig. 11. However, the reciprocal of $\left|\tilde{\omega}_{\tilde{\tau}}\right|$ goes to infinity when $\tilde{\omega}_{\tilde{\tau}}$ crosses the imaginary axis (dashed gray line in Fig. 11). A better estimate of the time scale in this region is provided by the reciprocal of the modulus of the complex eigenvalues (dashed black line in Fig. 11): therefore we define a new $\hat{\omega}_{\tilde{\tau}}$ as:

$$
\begin{aligned}
& \hat{\omega}_{\tilde{\tau}}=\tilde{\tau} \cdot J_{g} \cdot \tilde{\tau} \\
& \hat{\omega}_{\tilde{\tau}}=\left|\lambda_{1}\right|=\left|\lambda_{2}\right| \quad \text { if: } \lambda_{1,2} \text { are complex conjugates. }
\end{aligned}
$$

In Sec. 3.1, we noted that to identify the controlling scale, CSP performs two actions: (i) it evaluates the eigensystem, and (ii) applies the CSP criterion, Eq. (25), which involves providing a user-defined error threshold. In the TSR analysis, $\hat{\omega}_{\tilde{\tau}}$ closely follows the largest positive eigenvalue (light gray line) in P1, the largest negative eigenvalue (dark gray line) in P3a, and the smallest negative eigenvalue (light gray line) in P3b. Thus, $\hat{\omega}_{\tilde{\tau}}$ is able to track the controlling time scale at all times (see Figs. 9 and 11), and it achieves this goal without involving any userdefined error threshold.

An apparent advantage in using the reciprocal of $\left|\hat{\omega}_{\tilde{\tau}}\right|$ as an estimate of the driving time scale (dashed black line) is the smooth transition from the fast to the slow scale occurring in 
the time range $t \approx 0.2-0.45$. The CSP driving scale defined as $\tau_{M+1}$ will suddenly switch from the fast $(M=0)$ to the slow scale $(M=1)$ as soon as the fast mode amplitude satisfies Eq. (25).

\section{N-dimensional extension}

Extending to the $N$-dimensional case the definition of $\hat{\omega}_{\tilde{\tau}}$ given above for the $2 \mathrm{D}$ case, simply involves replacing the eigenvalue with its modulus in Eq. (20). The sign of the TSR is set according with the sign of the real part of the eigenvalue. Therefore, we propose computing the TSR as

$$
\bar{\omega}_{\tilde{\tau}}:=\sum_{i=1}^{N} \bar{W}_{i} \operatorname{sgn}\left(\operatorname{Re}\left(\lambda_{i}\right)\right)\left|\lambda_{i}\right|, \quad \bar{W}_{i}=\frac{W_{i}}{\sum_{j=1}^{N}\left|W_{j}\right|}
$$

with

$$
W_{i}:=\frac{f^{i}}{\mathrm{~g}} \frac{\boldsymbol{g} \cdot \boldsymbol{a}_{i}}{\mathrm{~g}} .
$$

Particular attention must be paid when the system of interest involves state variables having different units of measure (mole fractions vs. Kelvin). In this case, the evaluation of the inner products $\boldsymbol{g} \cdot \boldsymbol{a}_{i}$ and $\boldsymbol{a}_{j} \cdot \boldsymbol{a}_{i}$ in Eq. (21) require special care. Moreover, if $\boldsymbol{g}$ is an infinite dimensional vector field defined in some functional space (e.g., as in PDEs), then the definition of the inner product should be consistent with that of the functional space of interest. To overcome these difficulties, one can resort to the estimate of $\bar{\omega}_{\tilde{\tau}}$ obtained by taking the equality sign in Eq. (22), to yield this expression for the weights to use in definition (30):

$$
W_{i}:=\left(\frac{f^{i}}{\mathrm{~g}}\right)^{2} \text {. }
$$

Finally, when there are complex eigenvectors and eigenvalues, the definition needs to be properly modified as described in Appendix A.

\section{Participation Index of a Mode to the TSR}

The additive nature of definition (30) suggests introducing a Participation Index of the $i$-th mode to the TSR as:

$$
P_{\text {mode }_{i}}^{\bar{\omega}_{\tilde{\tau}}}=\operatorname{sgn}\left(\operatorname{Re}\left(\lambda_{i}\right)\right) \frac{\bar{W}_{i}\left|\lambda_{i}\right|}{\sum_{j=1}^{N}\left|\bar{W}_{i}\right| \lambda_{i}||}
$$

Modes with a large and positive/negative $P_{\text {mode }}^{\bar{\omega}_{\tilde{\tau}}}$ are the ones that contribute the most to the growth/decay of the most energy containing time scale. Next, the reactions that contribute the most to the development of the $\omega_{\tilde{\tau}}$ scale can be identified by resorting to the CSP participation index for the $k$-th reaction to these modes, $P_{\text {reaction }_{k}}^{\text {mode }_{i}}$.

For the reader's convenience, we recall the definition of the CSP participation index for the $k$-th reaction to the $m$-th mode in the following paragraph. Recall first that for a chemical kinetic problem the source term $g$ in Eq. (1) can be expressed as the sum of contributions from all the elementary reactions in the kinetic mechanism, so that it can be expressed as:

$$
\boldsymbol{g}=\sum_{k=1}^{2 N_{r}} \boldsymbol{S}_{k} r^{k}
$$

where $N_{r}$ is the number of reversible reactions, $S_{k}$ and $r^{k}$ are the stoichiometric vector and rate of progress of the $k$-th irreversible reaction, respectively.

Given this, the relative contribution of the $k$-th irreversible reaction to the amplitude of the $i$-th mode is measured by the CSP participation index defined as:

$$
P_{\text {reaction }_{k}}^{\text {mode }_{i}}=\frac{C_{k}^{i} r^{k}}{\sum_{k^{\prime}=1}^{2 N_{r}}\left|C_{k^{\prime}}^{i} r^{k^{\prime}}\right|} \quad i=1, N_{s}, k=1,2 N_{r}
$$

with

$$
C_{k}^{i}=\boldsymbol{b}^{i} \cdot \mathbf{S}_{k},
$$

where $N_{s}$ is the number of species, and $\boldsymbol{b}^{i}$ is the left eigenvector of the $i$-th mode.

To identify the reactions most contributing to the development of $\bar{\omega}_{\tilde{\tau}}$, we can introduce an index relating the $\mathrm{k}-$ th reaction to $\bar{\omega}_{\tilde{\tau}}, P_{\text {reaction }_{k}}^{\bar{\omega}_{\tilde{\tau}}}$, as the product of the participation index of the mode $i$ to the TSR, $P_{\text {mode }}^{\bar{\omega}_{\tilde{\tau}}}$, times the CSP participation index of the $\mathrm{k}$-th reaction to the $\mathrm{i}-$ th mode, $P_{\text {reaction }_{k}}^{\text {mode }_{i}}$, that is:

$$
P_{\text {reaction }_{k}}^{\bar{\omega}_{\tilde{\tau}}}:=P_{\text {mode }_{i}}^{\bar{\omega}_{\tilde{\tau}}} \times P_{\text {reaction }_{k}}^{\text {mode }_{i}}
$$

Note that the sum of the absolute values of $P_{\text {reaction }}^{\bar{\omega}_{\tilde{\tau}}}$ is not normalized to unity.

\section{TSR Analysis of Williams Model}

We are now ready to carry out the TSR analysis of the Williams model using the final definition of the TSR as given by Eq. (30). We will carry out the analysis by using both definitions of the weights as defined in (31) and (32) to point out their different behavior.

This system exhibits two main ignition modes named sub and supercritical when the parameter $\gamma$ is below and above the critical value of $\gamma_{c r}=\alpha-1$. When $\gamma<\gamma_{c r}=\alpha-1$, the rate at which the intermediates are consumed by the termination step is slower than the rate of production by chain branching; this determines a large and quick growth (mass explosion) of the intermediates. Instead, when $\gamma>\gamma_{c r}=\alpha-1$, the termination step is effectively able to keep the intermediates at very low values. This system has been studied by considering uncertain initial conditions and model parameters in [35].

\subsection{Supercritical Regime}

To study the Supercritical Regime of ignition, we set the parameters of the Williams model as follows : $\alpha=2, \epsilon=0.01$, $\gamma=0.5 \gamma_{c r}$, with $\gamma_{c r}=\alpha-1$. Setting $\gamma<\alpha-1$ implies that the termination step consumes the intermediate at a slower pace than its chain-branching production, this leading to a significant and fast growth of the intermediate. 
Figure 12 portraits the isocontour plot of the field of the fast mode amplitude $\left(f_{\text {fast }}=\boldsymbol{b}_{\text {fast }} \cdot \boldsymbol{g}\right)$, where $\boldsymbol{g}$ is the rhs of Eq. (26) and $\boldsymbol{b}_{\text {fast }}$ is the left eigenvector corresponding to the fast right eigenvector of the Jacobian matrix of $\boldsymbol{g}$. A leading order approximation of the SIM of the dynamical system (26) is provided by the iso contour line of $f_{\text {fast }}=0$, which is the boundary separating the regions (in dark/light gray shade) of positive/negative $f_{\text {fast }}$, respectively. It is apparent that in the supercritical regime, the trajectory (black solid line) reaches the SIM only very late during its time evolution, meaning that for most of the time the dynamics is off the SIM.

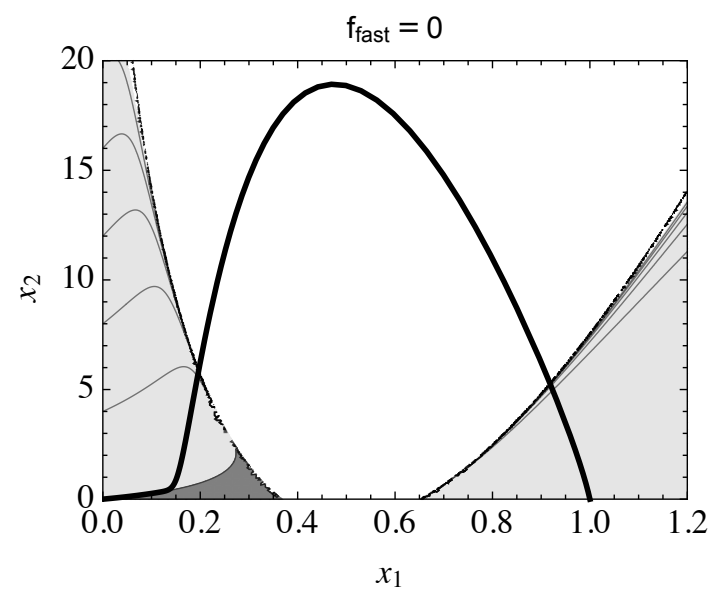

Figure 12: Supercritical regime. Fast mode amplitude of Williams model; dark/light gray shade indicates positive/negative value of $f_{\text {fast }}$; the trajectory is marked by the black solid line; isocontour lines refer to constant fast mode amplitudes.

To understand why, we plot the isocontour plot of the fields of the real part of the fast (top figure) and slow (right figure) eigenvalues in Fig. 13. Dark/light gray shade indicates positive/negative value of real part of the fast (top) and slow (bottom) eigenvalues; white region denotes a complex eigenvalue. For the prescribed initial conditions, the trajectory starts where both eigenvalues have positive real part, then enters the region of complex eigenvectors, and finally proceeds towards the SIM and the fixed point in the region where both eigenvalues have negative real part (as already discussed in Sec.3).

We compare in Fig. 14 (top), the time evolution of the real part of eigenvalues (dark/light gray lines), with the TSR computed according with formulas (31) (dashed black line) and (32) (long dashed black line). In this case, there is no qualitative difference and only a moderate quantitative difference between the two TSR definitions.

The absolute value of the two modal amplitudes is shown in Fig. 14 (bottom). When the fast amplitude (light gray line) becomes smaller than the slow amplitude (dark gray line), then the trajectory approaches the SIM (defined indeed by $f_{\text {fast }}=0$ ). This is what TSR indicates (in Fig. 14 (top)) when the TSR lines switch from being first coincident with the fast mode eigenvalue to being coincident with the slow mode eigenvalue.
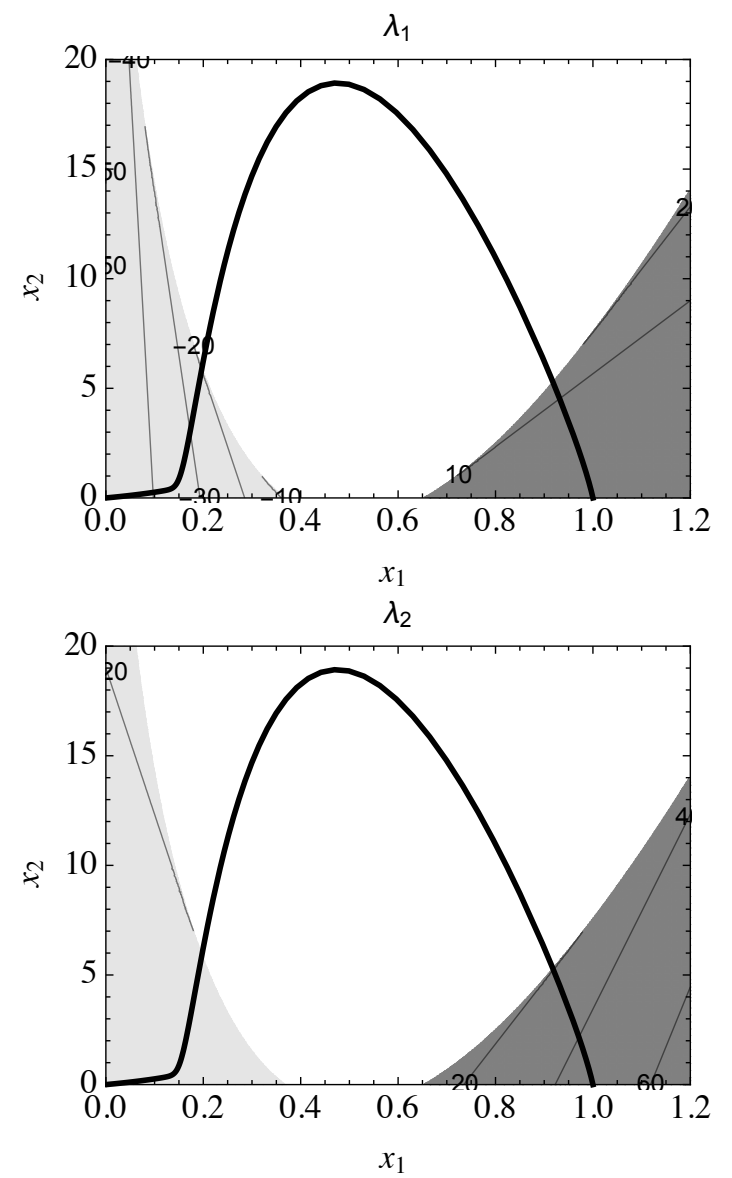

Figure 13: Supercritical regime. Dark/light gray shade indicates positive/negative value of real part of the fast (top) and slow (bottom) eigenvalues; white region denotes a complex eigenvalue; isocontour lines refer to constant eigenvalue magnitude.

\subsection{Subcritical Regime}

To study the Subcritical Regime of ignition, we set the parameters of the Williams model as follows: $\alpha=2, \epsilon=0.01, \gamma$ $=1.5 \gamma_{c r}$, with $\gamma_{c r}=\alpha-1$.

Figure 15 indicates that the trajectory lies completely in the region of eigenvalues with negative real part. This justifies naming the regime as subcritical. Note that the white region denoting a complex eigenvalue is now at the right of the trajectory. Thus, the whole dynamics is dominated by dissipative (contractive) processes. Indeed, setting $\gamma>\alpha-1$ implies that the termination step consumes the intermediate at a faster pace than its chain-branching production, this preventing a significant and fast growth of the intermediate.

The effect of the dissipative (contractive) processes is (i) to force the trajectory to quickly approach the SIM (Fig. 16), (ii) the quick collapse of the small circular probe drawn about the initial condition (Fig. 17).

The parameter $\epsilon<1$ controls the degree of stiffness (time scale gap between fast and slow scales) of the system, and is the responsible of the strong curvature experienced by the trajectory while exhausting its fast transient and in its approach to the SIM. In fact, at the fixed point $(0,0)$ the two eigenvalues 

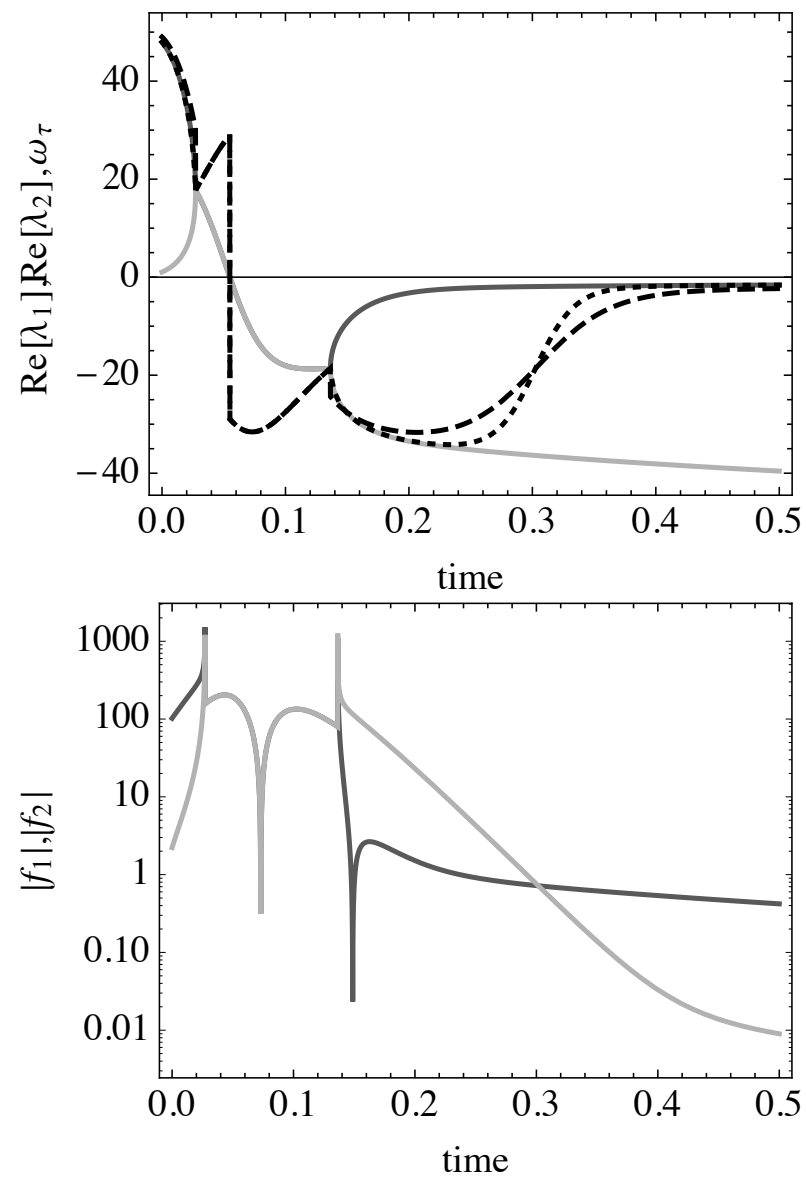

Figure 14: Supercritical regime. (top) Real part of eigenvalues (dark/light gray lines); TSR computed according with formula (31) (long dashed black line) and (32) (dashed black line); (bottom) absolute value of the two modal amplitudes.

take the values $\lambda_{\text {slow }}=-1$ and $\lambda_{\text {fast }}=-\gamma / \epsilon$, so that their ratio is simply $\lambda_{\text {slow }} / \lambda_{\text {fast }}=\epsilon / \gamma<1$ for $\gamma \sim O(1)$ and $\epsilon<1$.

Figure 18 (top) indicates that there is a qualitative and quantitative difference between the two definition of the TSR. In fact, the TSR computed with formula (31) (long dashed line) exhibits a significant overshoot with respect to the two eigenvalues, so that it becomes faster than the fast eigenvalue, and slower (even positive) with respect to the slow eigenvalue. Instead the TSR computed with formula (32) (dashed line) exhibits no overshoot and proceeds monotonously from the fast to the slow eigenvalue. We will discuss in Sec. 11 that this difference is caused by the contribution of the direction cosines, which perceive the changes in the relative orientation between the eigenvectors and the vector field of the model problem (which is by definition, always tangent to the trajectory). Eventually, we will show that the TSR computed with formula (31) is sensitive to the strong curvature experienced by the trajectory while exhausting its fast transient and in its approach to the SIM. Note for future reference, that the angle between the two eigenvectors of the Williams model in the subcritical regime becomes minimal in the region of strongest curvature of the trajectory (Fig. 19).
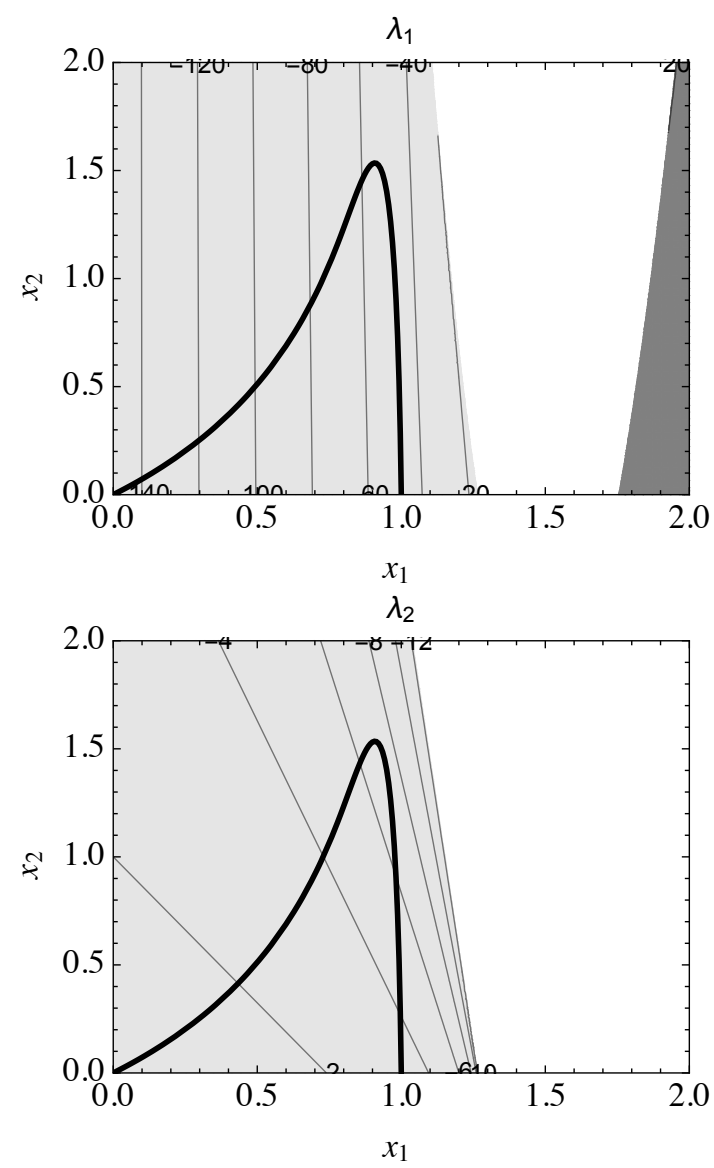

Figure 15: Subcritical regime. Dark/light gray shade indicates positive/negative value of real part of the fast (top) and slow (bottom) eigenvalues; white region denotes a complex eigenvalue.

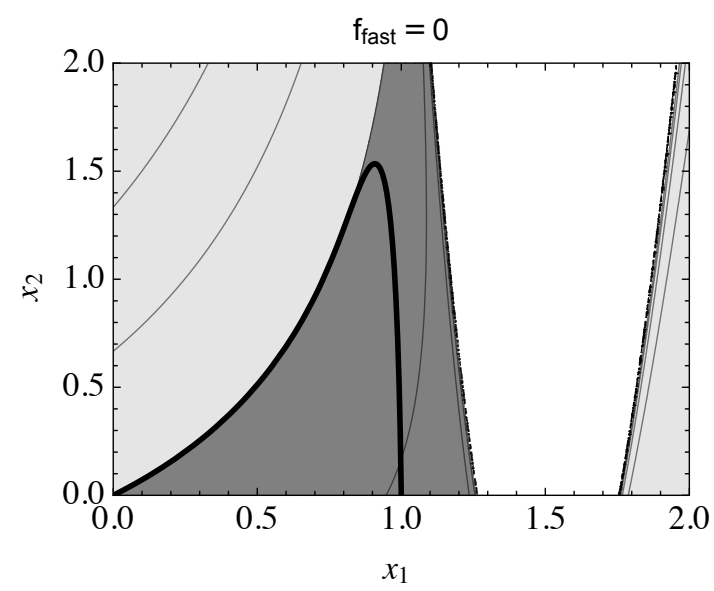

Figure 16: Subcritical regime. Fast mode amplitude of Williams model; dark/light gray shade indicates positive/negative value of $f_{\text {fast }}$; the trajectory is marked by the black solid line; isocontour lines refer to constant fast eigenmode magnitude.

\section{The Non-Isothermal Semenov Model}

A thermal explosion model can be derived starting from the system studied by Semenov in $[33,34]$. The Semenov model 


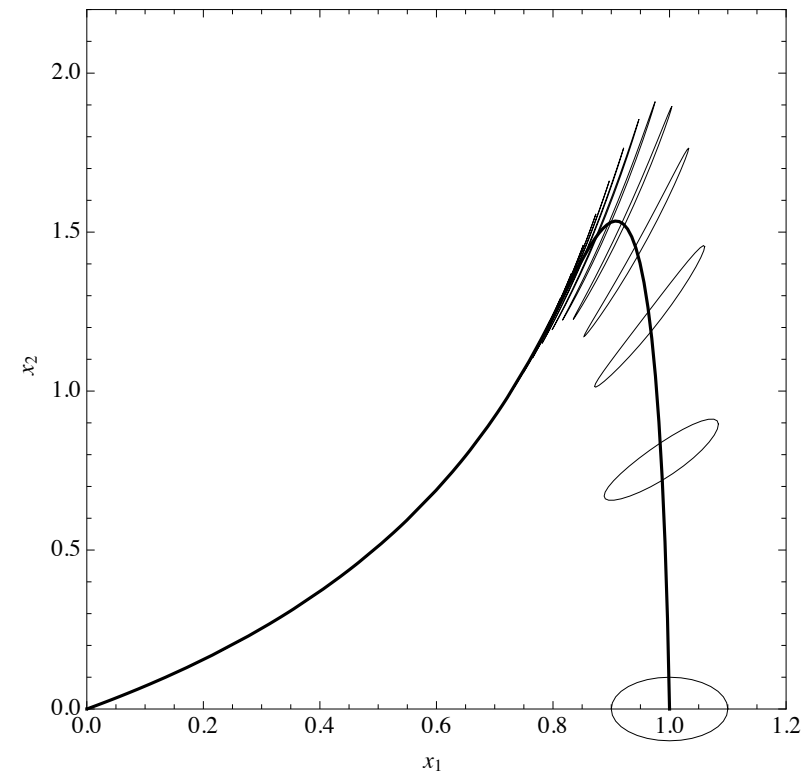

Figure 17: Subcritical regime. Evolution of a small circle drawn about the initial condition and advected in time by the dynamical system (axis have different scales); the trajectory is marked by the black solid line.
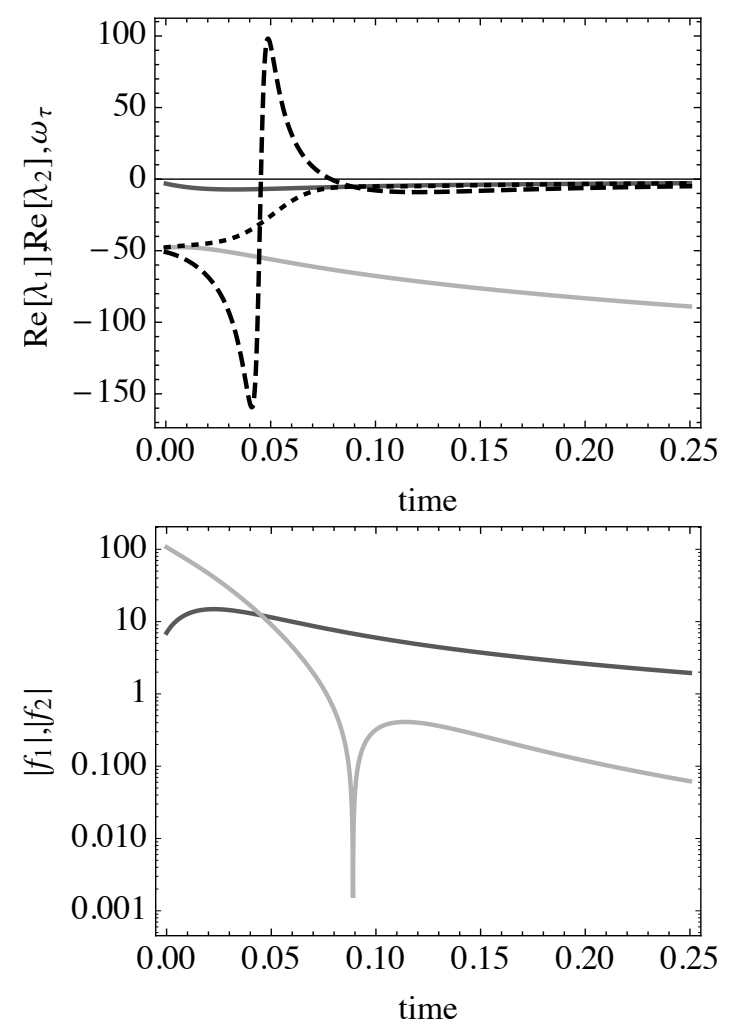

Figure 18: Subcritical regime. (top) Real part of eigenvalues (dark/light gray lines); TSR computed according with formula (31) (long dashed black) and (32) (dashed black); (bottom) absolute value of the two modal amplitudes.

describes the non-isothermal explosion process which might occur in a reservoir where combustion generates heat which is released to the ambient through non-adiabatic walls.

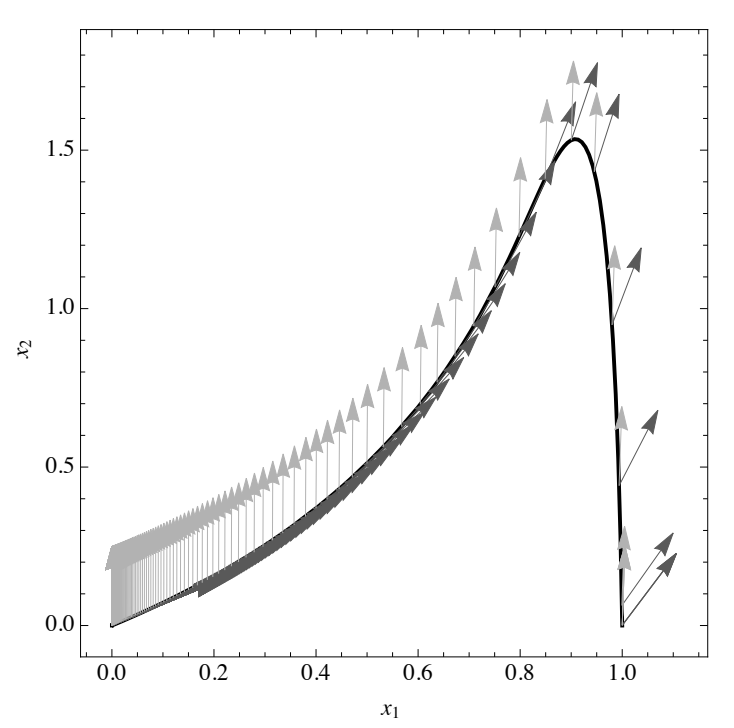

Figure 19: Subcritical regime. Evolution of eigenvectors of Williams model in phase space; fast/slow eigenvectors plotted as dark/light gray arrows.

In this case, the system is purely controlled by an exponential non linearity. A comprehensive dynamical analysis of this model has been carried out in [32].

The state variables of the model problem as defined by Semenov are the temperature $T$ of the reactive mixture filling the reservoir, and the amount of fuel described by its molar concentration $C_{f}$. The reservoir is therefore a non-adiabatic system, where there are heat losses modeled as $q_{\text {wall }}(T)=h_{T} S\left(T-T_{w}\right)$, and where fuel can leave the system by absorption at the walls, as modeled by $\dot{m}_{f u e l}\left(C_{f}\right)=h_{f} S C_{f}$. The kinetics is modeled as a one-step reaction Fuel $\rightarrow$ Products, with a reaction constant retaining the exponential dependence on temperature $K(T)=$ $B_{0} e^{-\frac{E_{a}}{R_{u} T}}$, and a reaction rate of the form $W\left(T, C_{f}\right)=K(T) C_{f}^{1}$, that is, the one step kinetics is assumed to have a reaction order 1 with respect to the fuel. The initial temperature of the fuel is $T_{0}$ and is assumed at equilibrium with the isothermal walls kept at the temperature $T_{w}$. The initial fuel concentration is $C_{f, 0}$, The volume and surface, $V$ and $S$, of the reservoir are constant in time. The specific heat of the fuel and products, $C_{v}$, the density of the reactive mixture, $\rho$, the heat of combustion, $Q_{F}$, are all constant in time. The activation energy and the steric factor of the one-step kinetics are $E_{a}$ and $B_{0}$, respectively, the universal gas constant is $R_{u}$. The energy and mass balance for this system reads:

$$
\begin{aligned}
V \rho C_{v} T^{\prime} & =+V Q_{F} W\left(T, C_{f}\right)-q_{\text {wall }}(T) \\
V C_{f}^{\prime} & =-V W\left(T, C_{f}\right)-\dot{m}_{\text {fuel }}\left(C_{f}\right)
\end{aligned}
$$

This set can be cast in non dimensional form by introducing 
the following parameters

$$
\begin{aligned}
t_{c} & =\frac{\rho C_{v}}{h_{t} S / V} \\
\alpha & =\frac{\rho C_{v} h_{f}}{h_{t}} \\
\epsilon & =\frac{R_{u} T_{w}}{E_{a}} \\
\gamma & =\frac{\rho C_{v} T_{w}}{C_{f, 0} Q_{F}} \\
\delta & =\frac{B_{0} t_{c} e^{-\frac{1}{\epsilon}}}{C_{f, 0} \gamma \epsilon}
\end{aligned}
$$

Next, we introduce the non dimensional state variables, reduced temperature and fuel concentration, $\theta$ and $\varphi$, and time $\tau$ :

$$
\begin{aligned}
\tau & =\frac{t}{\frac{\rho C_{v}}{h_{t} S / V}} \\
\theta & =\frac{E_{a}(T-T w)}{R_{u} T_{w}{ }^{2}} \\
\varphi & =\frac{C_{f}}{C_{f, 0}}
\end{aligned}
$$

We cast the non dimensional exponential term as

$$
\tilde{W}(\theta, \varphi)=\left(B_{0} e^{-\frac{1}{\epsilon}}\right) e^{\frac{\theta}{1+\epsilon \theta}} \varphi
$$

By carrying out all the transformation on (38) required by the nondimensionalization process, and after having renamed $x_{1}=\theta$ and $x_{2}=\varphi$ for coherence with the other model problem discussed in this work, we can write the final form of the Semenov model as:

$$
\begin{aligned}
\delta \frac{d x_{1}}{d \tau} & =+\Omega\left(x_{1} x_{2}\right)-\frac{x_{1}}{\delta} \\
\delta \frac{d x_{2}}{d \tau} & =-\gamma \epsilon \Omega\left(x_{1} x_{2}\right)-\alpha \frac{x_{2}}{\delta} \\
x_{1}(0) & =1 \quad x_{2}(0)=0
\end{aligned}
$$

with

$$
\Omega\left(x_{1} x_{2}\right)=x_{2} e^{\frac{x_{1}}{\epsilon x_{1}+1}}
$$

A comprehensive dynamical analysis of this model has been carried out in [32]. A bifurcative analysis of (39) indicates that this system exhibits two main ignition modes named sub and supercritical when the parameter $\delta$ is below and above the value of $\delta_{c r}$ that satisfies the following equation:

$$
\epsilon^{-\frac{1}{\beta}} \delta_{c r}-\epsilon=0
$$

The small parameters $\alpha$ and $\epsilon$ control the degree of stiffness of the problem. Changing $\alpha$ changes the SIM of the system, whereas $\epsilon$ does not influence the SIM definition.

\subsection{Supercritical Regime}

The set of parameters chosen to discuss a supercritical explosion in the Semenov model are $\epsilon=0.01, \gamma=0.01, \alpha=$ $0.001, \delta=1.4(1 / e)>\delta_{c r}$.

Inspection of Fig. 20 (top), 21, and 22 show that the supercritical trajectory (i) starts in a region where both eigenvalues have a negative real part; (ii) enters the (white) region of complex eigenvalue where both eigenvalues have first a negative and then a positive real part; (iii) enters a region where both eigenvalues are real and positive; (iv) transits back in the region of complex eigenvalue; ( $v$ ) enters at about time $t=35$ in the region where both eigenvalues are real and negative.

The evolution of the small circular probe drawn about the initial condition shown in Fig. 20 (bottom) features a significant growth only after the trajectory enters the region where both eigenvalues have a positive real part. On the other hand, as indicated in Fig. 20 (top), the circular probe, past time $t=35$, becomes negligibly small when the trajectory lands in the SIM defined by equation $f_{\text {fast }}=0$.
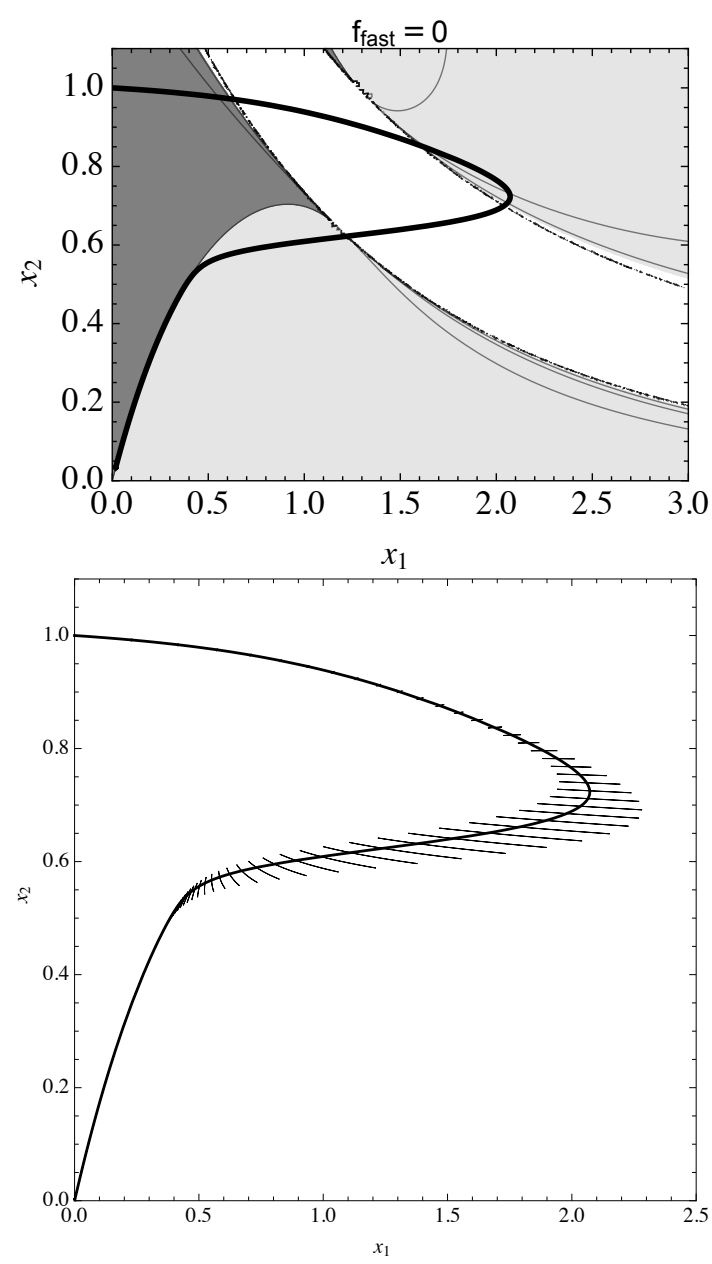

Figure 20: Supercritical regime. (top) Fast mode amplitude of Semenov model; dark/light gray shade indicates positive/negative value of $f_{\text {fast }}$; the trajectory is marked by the black solid line; isocontour lines refer to constant fast eigenmode magnitude. (bottom) Evolution of the small circular probe drawn about the initial condition. 

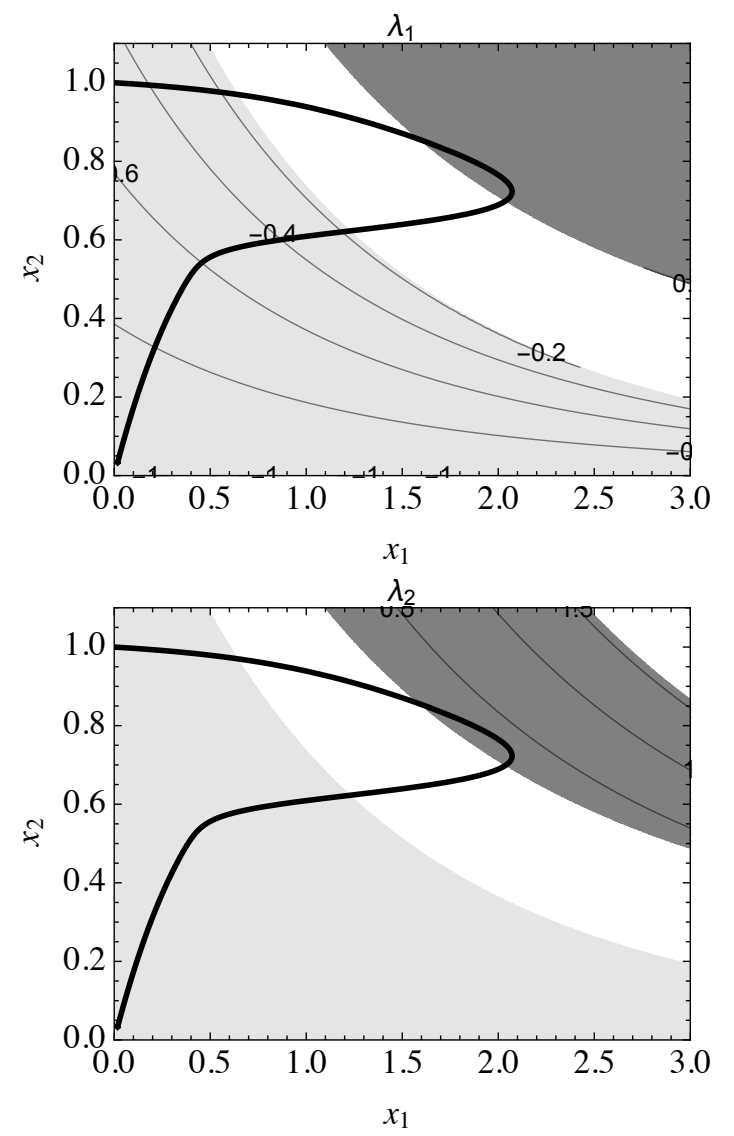

Figure 21: Supercritical regime. Dark/light gray shade indicates positive/negative value of real part of the fast (top) and slow (bottom) eigenvalues; white region denotes a complex eigenvalue; isocontour lines refer to constant eigenvalue magnitude.

Inspection of Fig. 22 indicates quite a number of differences between the time evolution of the TSR computed according with formula (31) (long dashed black) and (32) (dashed black). Note that the black TSR line before time $\mathrm{t}=2$, follows a path which is close to the slow (black solid line) negative eigenvalue, because the slow mode amplitude is larger than the fast (Fig. 22 (bottom)). However, the long dashed line is practically coincident with the largest of the two negative eigenvalues. This suggests that the direction of the slow eigenvector being quite different from the one of the vector field is able to cancel the contribution of the slow mode to the TSR computed according with formula (31).

The long dashed and dashed black lines also differ during the time period $(7<\mathrm{t}<12)$ where both eigenvalues are real and positive, and where both eigenvalues are real and negative $(\mathrm{t}$ $>17$ ). This behavior, which is sensed by the long dashed line and not sensed by the dashed black line, can be attributed to the strong curvature experienced by the trajectory, where both eigenvectors approximately point towards the same direction as confirmed by Fig. 23. It is also at later times that the long dashed, the dashed, and the light gray lines merge, all identifying the slowest real and negative eigenvalue as the scale taking the system to the fixed point.
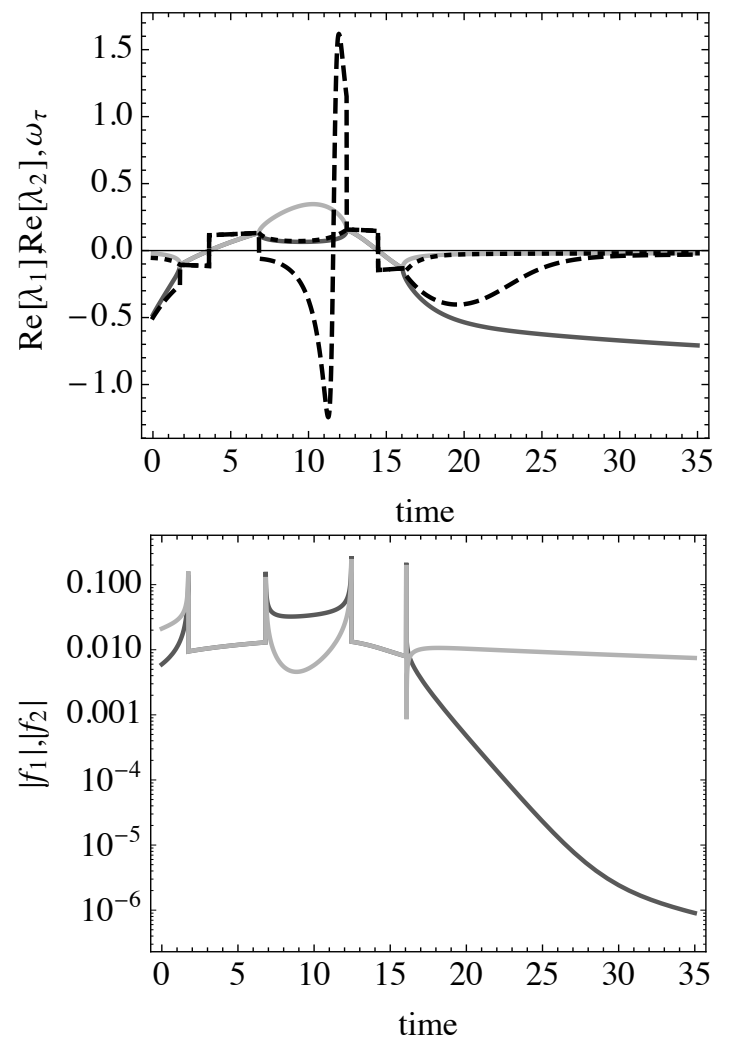

Figure 22: Supercritical regime. (top) Real part of eigenvalues (dark/light gray lines); TSR computed according with formula (31) (long dashed black) and (32) (dashed black); (bottom) absolute value of the two modal amplitudes.

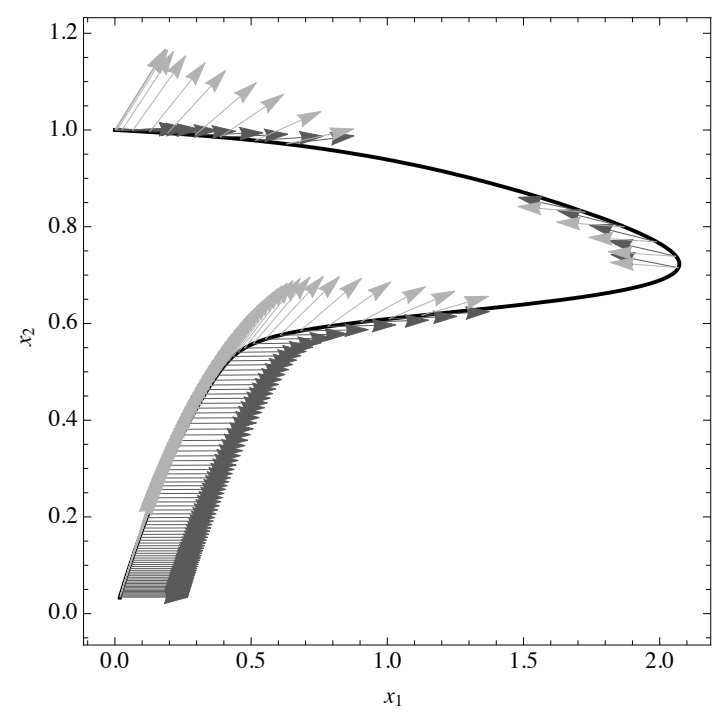

Figure 23: Supercritical regime. Evolution of eigenvectors of Semenov model in phase space; fast/slow eigenvector = dark/light gray arrows.

\subsection{Subcritical Regime}

The set of parameters chosen to discuss a subcritical explosion in the Semenov model are $\epsilon=0.01, \gamma=0.01, \alpha=$ $0.001, \delta=1.2(1 / e)<\delta_{c r}$.

Inspection of Fig. 24, 26, and 27 show that the subcritical tra- 
jectory is fully contained in the region where both eigenvalues are real and negative, which justifies attributing the subcritical adjective to this

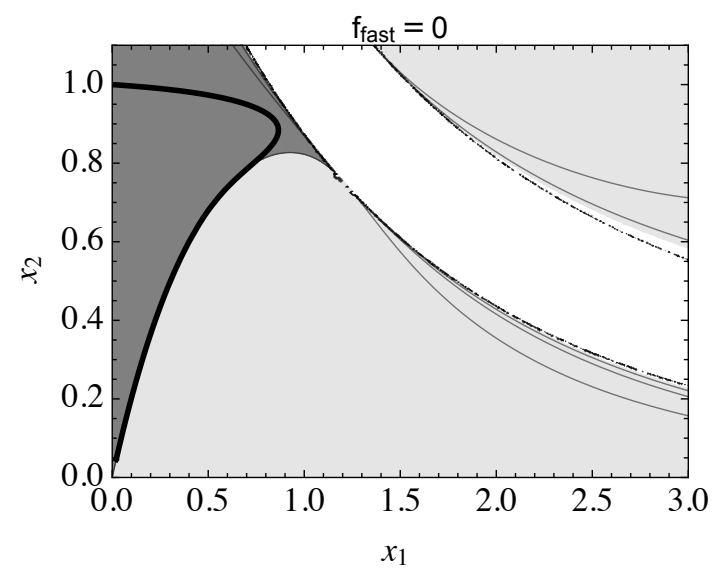

Figure 24: Subcritical regime. Fast mode amplitude of Semenov model; dark/light gray shade indicates positive/negative value of $f_{\text {fast }}$; the trajectory is marked by the black solid line; isocontour lines refer to constant fast eigenmode magnitude.

The evolution of the small circular probe drawn about the initial condition shown in Fig. 25 features a progressive contraction as the trajectory is quickly attracted towards the SIM defined by equation $f_{\text {fast }}=0$.

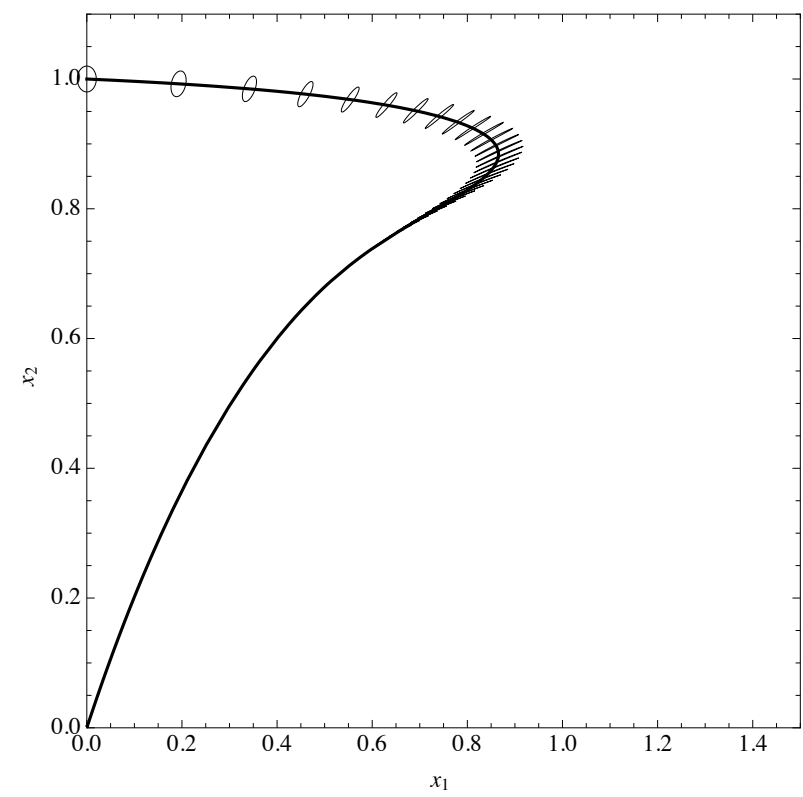

Figure 25: Subcritical regime. Evolution of the small circular probe drawn about the initial condition.

Inspection of Fig. 27 indicates a significant difference between the time evolution of the TSR computed according with formula (31) (long dashed black) and (32) (dashed black), which is qualitatively similar to what was observed earlier in the non-normal tunable linear model. Note that the black TSR
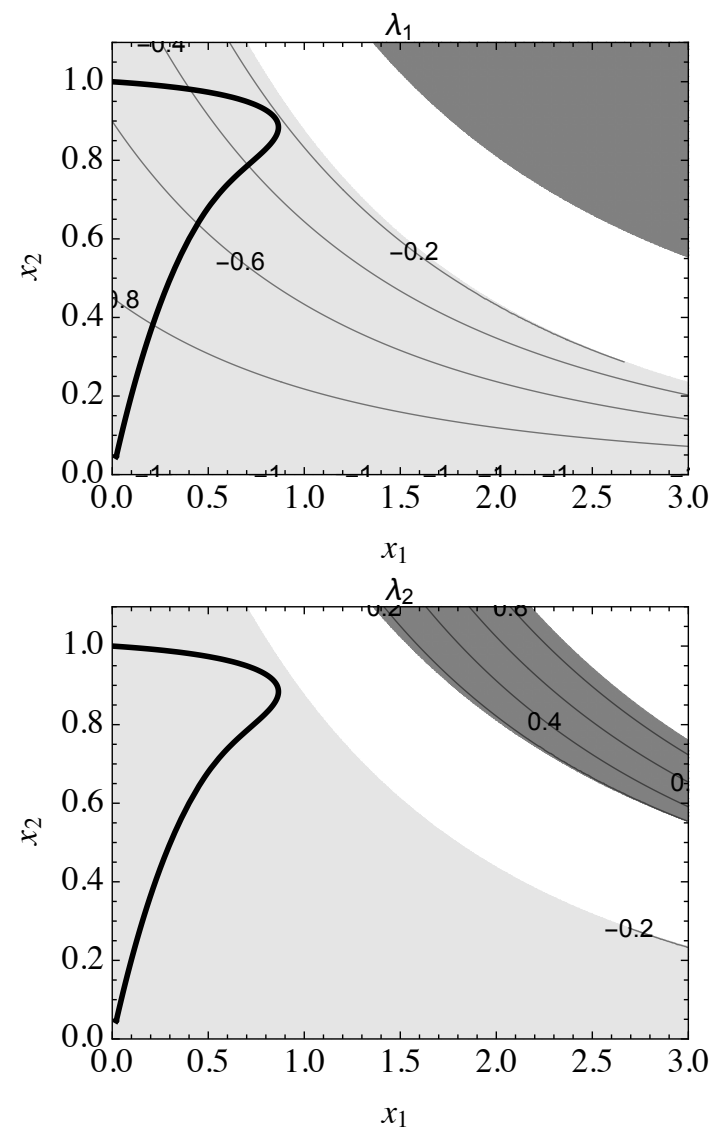

Figure 26: Subcritical regime. Dark/light gray shade indicates positive/negative value of real part of the fast (top) and slow (bottom) eigenvalues; white region denotes a complex eigenvalue; isocontour lines refer to constant eigenvalue magnitude.

line at very early times follows a path which at all times lies close to the slow (black solid line) negative eigenvalue; instead, the long dashed black line is initially close to the fast (dark gray line) negative eigenvalue, and later on it switches towards the slow (light gray line) negative eigenvalue. The transition between the two scales is accompanied by the overshoot already observed elsewhere and associated with the near co-linearity of the two eigenvectors in the region of strong curvature of the trajectory as noticeable in Fig. 28.

According to Fig. 27 (bottom), the amplitude of the slow mode is uniformly larger than that of the fast. However, at early times, the amplitude of the fast mode is not negligible, and makes the fast mode the controlling one during the transient phase (before time $\mathrm{t} \sim 12$ ) that brings the system from the initial condition to the SIM.

All these observations suggest that the direction of the slow eigenvector is quite different from the one of the vector field as confirmed by Fig. 28. 

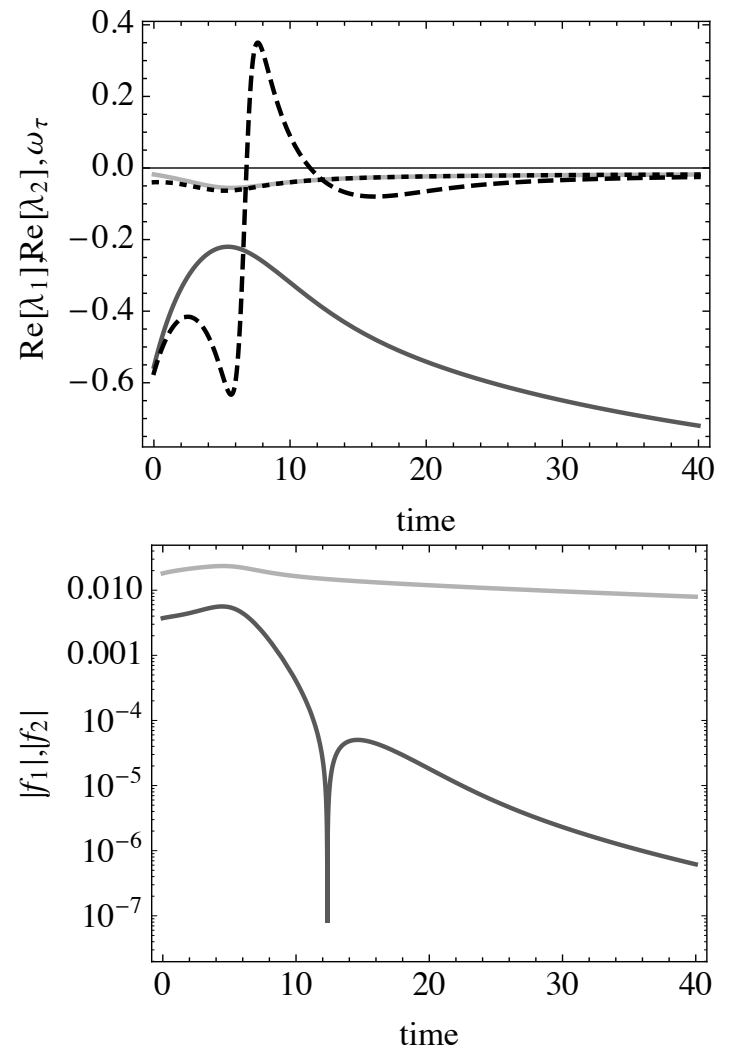

Figure 27: Subcritical regime. (top) Real part of eigenvalues (dark/light gray lines); TSR computed according with formula (31) (long dashed black line) and (32) (dashed black line); (bottom) absolute value of the two modal amplitudes.

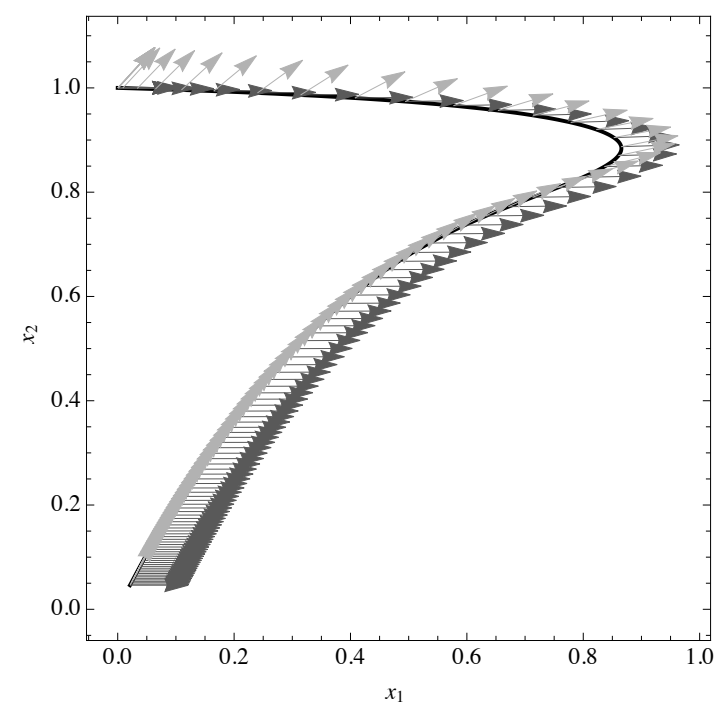

Figure 28: Subcritical regime. Evolution of eigenvectors of Semenov model in phase space; fast/slow eigenvector = dark/light gray arrows.

\section{The Branched-Chain Reactions Non-Isothermal Kapila Model}

The Williams model mimics the dynamics of an isothermal branched-chain reactions system, characterized by a quadratic (polynomial) non linearity. It is also of interest to study the dynamics when branched-chain reactions are combined with heat production to make the system non-isothermal.

Kapila in 1978 developed a model problem with these characteristics and carried out its full asymptotic study as reported in [22]. In Kapila's model, the relevant nonlinearities are the result of the product of an exponential times a quadratic nonlinearity (Eq. 47).

A brief summary of the model problem is outlined in the following for the reader convenience.

Let us consider a constant volume batch reactor filled with a reactive mixture containing a single reactant $\mathrm{A}$. The reactant $\mathrm{A}$ is converted into products through reaction with a single active intermediary $\mathrm{C}$ according to a second-order chain-branching step. The sole recombination process occurs at the walls of the vessel according to first order kinetics.

$$
\begin{aligned}
& R \# 1: A+C \rightarrow(1+n) C+\text { products } \\
& R \# 2: \quad C \rightarrow \text { stable species }
\end{aligned}
$$

According to Kapila [22], the assumptions taken to define the model are: (i) a small concentration of chain carriers is already present in the starting mixture (rather than postulating an initiation reaction, which is usually far slower than the other two steps and therefore unlikely to influence the overall reaction rate), (ii) the propagation step $\mathrm{R} \# 1$ is assumed to have a strongly temperature-dependent rate, i.e., a large activation energy, but is considered to be thermally neutral, (iii) the overall reaction is exothermic with the entire heat being liberated in the termination step R\#2, and (iv) the system is spatially homogeneous.

With these assumptions, the mathematical equations governing the reactive system include the rate equations for the production/consumption of species and the energy conservation $[20,22]$, which are written using with the following definitions: $n$ the branching factor; $\mathrm{W}_{a}, \mathrm{~W}_{b}, \mathrm{~W}_{c}$, the molecular weights of species A, B, and C, respectively; $\alpha=\left(\mathrm{Q} /\left(\mathrm{C}_{p} \mathrm{~T}_{o}\right)\right)\left(\mathrm{n} \mathrm{W}_{c} / \mathrm{W}_{a}\right)$ $\mathrm{Y}_{A, o}$, with $\alpha$, the non dimensional initial fuel; $\beta=\left(\mathrm{Q} /\left(\mathrm{C}_{p} \mathrm{~T}_{o}\right)\right)$ $\left(\mathrm{n} \mathrm{W}_{c} / \mathrm{W}_{a}\right)\left(\mathrm{E}_{1} /\left(\mathrm{RT}_{o}\right)\right) \mathrm{Y}_{C, o}$, with $\beta$ the non dimensional initial intermediate; $\epsilon=\mathrm{RT}_{o} / \mathrm{E}_{1}$, with $\epsilon \ll 1$, the small parameter controlling the heat release rate; $\lambda=\left(\mathrm{B}_{1} \rho \mathrm{C}_{p} \mathrm{~T}_{o}\right) /\left(\mathrm{B}_{2} \mathrm{~W}_{c} \mathrm{Q}\right)$, with $\lambda$ the non dimensional ratio between propagation and termination reaction rates; $\rho$, the density of the mixture; $\mathrm{B}_{1}, \mathrm{~B}_{2}$ are the pre-exponential factors and $E_{1}, E_{2}$ the activation energies of the reaction steps $R \# 1$ and $R \# 2$, respectively ( $E_{2}$ is taken to be zero in accordance with assumption (ii) ); $Q$ is the heat release per unit mass; $R$ the universal gas constant; $\mathrm{C}_{p}$ the specific heat at constant pressure, and $t$ the time. For simplicity of the analysis, it is assumed that $\mathrm{C}_{p}$ and the $\mathrm{B}_{i}$ are constant.

The state variables are non dimensionalized as follows: $\mathrm{Y}_{A}$ $=\left(\mathrm{C}_{p} \mathrm{~T}_{o} / \mathrm{Q}\right)\left(\mathrm{W}_{a} /\left(\mathrm{n} \mathrm{W}_{c}\right)\right) y$, with $y$ the non dimensional fuel mass fraction, $(\mathrm{A}) ; \mathrm{Y}_{C}=\left(\mathrm{C}_{p} \mathrm{~T}_{o} / \mathrm{Q}\right) z$, with $z$ the non dimensional intermediate mass fraction, $(\mathrm{C}) ; \mathrm{T}=\mathrm{T}_{o} \theta$, with $\theta$ the non dimensional temperature; $\mathrm{t}=\tau / B_{2}$, with $\tau$ the non dimensional time.

The mathematical equations in non dimensional form read: 


$$
\begin{aligned}
\frac{d y}{d \tau} & =-\Omega(y, z, \theta) \\
\frac{d z}{d \tau} & =+\Omega(y, z, \theta)-z \\
\frac{d \theta}{d \tau} & =z \\
y(0) & =\alpha, \quad z(0)=\epsilon \beta, \quad \theta(0)=1
\end{aligned}
$$

with

$$
\Omega(y, z, \theta)=\lambda(\alpha, \beta) y z e^{\frac{1}{\epsilon}-\frac{1}{\epsilon \theta}}
$$

where $\lambda(\alpha, \beta):=\lambda_{0} \lambda_{\text {critic }}(\alpha, \beta) \sim O(1)$, with $\lambda_{\text {critic }}(\alpha, \beta):=$ -ProductLog $\left[-e^{\frac{-1-\beta}{\alpha}}\right]$, measures the speed of the propagation reaction relative to that of the termination reaction on an $\mathrm{O}(\exp (1 / \epsilon))$ scale. According to Kapila, we define as being "supercritical" all the solutions generated by taking $\lambda>\lambda_{\text {critic }}$, that is $\lambda_{0}>1$.

\section{TSR Analysis of the planar Kapila Model}

Kapila's original model is a three dimensional system which can be reduced to a two dimensional system, completely equivalent to the full three dimensional system, if one replace the differential equation for the time rate of change of the fuel (43) with the exact integral expression (48) expressing the conservation of absolute enthalpy in an adiabatic system:

$$
y+z+\theta=1+\alpha+\beta \epsilon
$$

This way the fuel mass fraction $y$ can be expressed as a function of the intermediate mass fraction $z$ and the mixture temperature $\theta$. For clarity, we introduce the state variables $\mathrm{x}_{1}, \mathrm{x}_{2}$ as follows:

$$
x_{1}=z, \quad x_{2}=\theta
$$

to yield a two dimensional system, equivalent to the full three dimensional system, defined as:

$$
\begin{aligned}
\frac{d x_{1}}{d \tau} & =+\Omega\left(1+\alpha+\beta \epsilon-x_{1}-x_{2}, x_{1}, x_{2}\right)-x_{1} \\
\frac{d x_{2}}{d \tau} & =x_{1} \\
x_{1}(0) & =\beta \epsilon, x_{2}(0)=1
\end{aligned}
$$

The dynamic significance of the model parameters $\epsilon, \lambda_{0}, \alpha, \beta$ is as follows; $\epsilon$ affects the degree of stiffness of the model but changing its value does not change the Slow Invariant Manifold (SIM) of the system; $\lambda_{0}$ both affects the degree of stiffness of the model and changing its value does change the SIM; $\alpha$ is TBD; $\beta$ is TBD.

The combination of the exponential and the quadratic nonlinearity in Kapila's model produces a rich variety of dynamical behavior. The most significant finding of immediate interest to the discussion on ignition is the observation that, differently from Williams' model, the positive eigenvalue can evolve into a negative one without (necessarily) crossing a region of complex eigenvalues.

To become convinced of this circumstance, we plot in Fig. 29 the field of the largest (with sign) eigenvalue for the selection of parameters $\epsilon=0.1, \lambda_{0}=1.4, \alpha=1.0, \beta=0.01 / \epsilon$.

The dark/light gray shade coding denote the region where the largest (with sign) eigenvalue has positive/negative real part. The "islands" in the landscape where the eigenvalues are complex are colored in white.
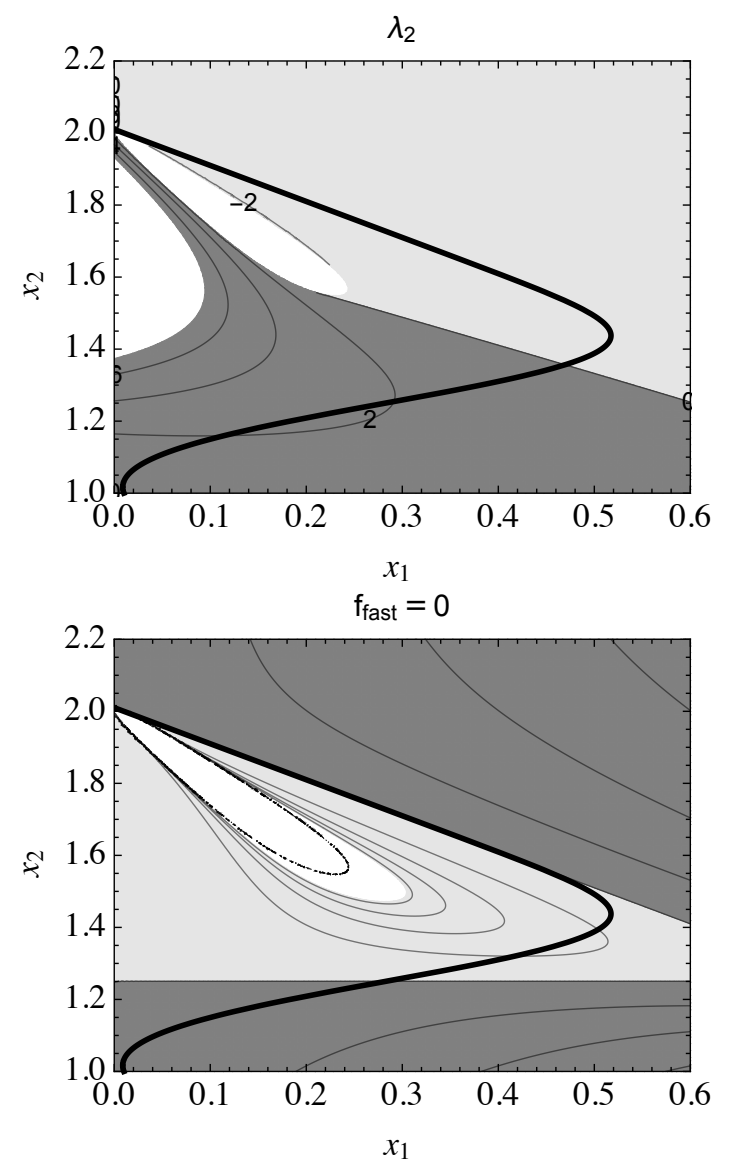

Figure 29: Evolution of the trajectory in the phase space without crossing a region of complex eigenvalues $\left(\epsilon=0.1, \lambda_{0}=1.4, \alpha=1.0, \beta=0.01 / \epsilon, x_{1}^{0}=\beta \epsilon=\right.$ $0.01, x_{2}^{0}=1.0$ ); (top) isocontour lines refer to constant fast eigenvalue magnitude; (bottom) isocontour lines refer to constant fast eigenmode magnitude.

In Fig. 29, the trajectory obtained with the set of parameters indicated here above and with initial conditions $x_{1}^{0}=$ $0.01, x_{2}^{0}=1.0$ is the solid black line. It can be easily appreciated (Fig. 29 (top)) that the trajectory moves from a dark to a light gray region without crossing the white island, meaning that the positive eigenvalue evolves into a negative one without crossing the region of complex eigenvalues. It is also apparent (Fig. 29 (bottom)) that the trajectory quickly approaches the SIM (this is so because with $\epsilon=0.1$ the system is quite stiff).

In Fig. 30 (top), we plot the time evolution of the two eigenvalues (dark and light gray lines) and of the TSR computed according with formula (31) and (32), (long dashed and dashed 
black lines, respectively). Note that both estimates of the TSR track the largest (in sign) slow eigenvalue before time $t=13.2$, whereas past time $t=13.2$ they track the smallest in sign fast eigenvalue. This development requires an explanation.
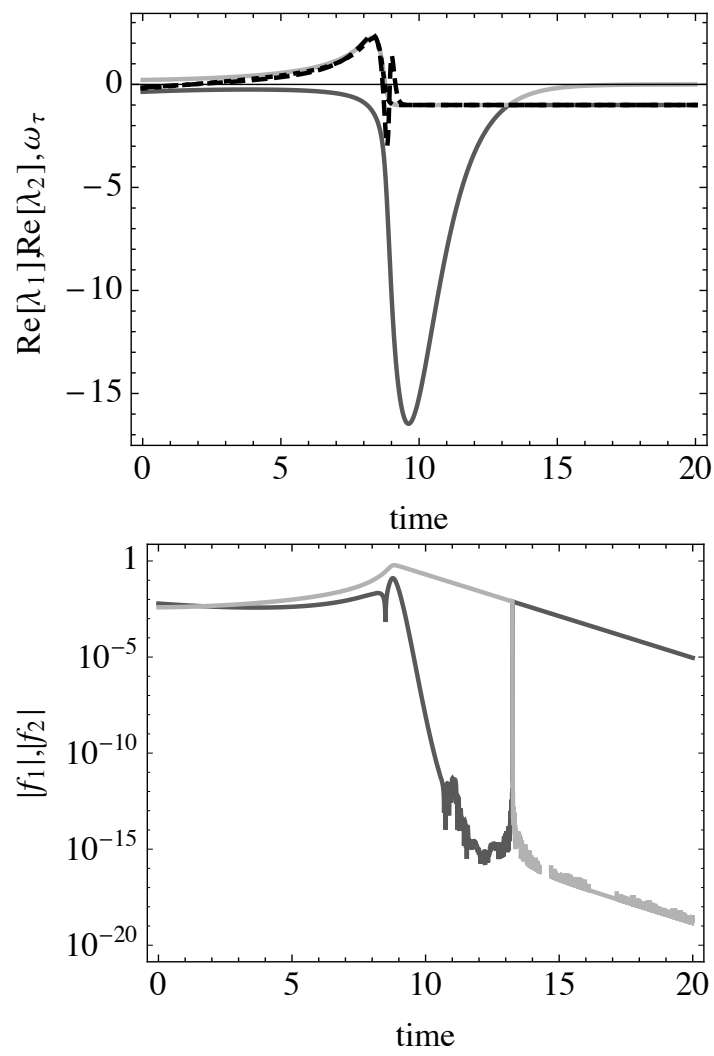

Figure 30: Time evolution of the TSR without crossing a region of complex eigenvalues ( $\epsilon=0.1, \lambda_{0}=1.4, \alpha=1.0, \beta=0.01 / \epsilon, x_{1}^{0}=\beta \epsilon=0.01, x_{2}^{0}=1.0$ ) (top) Real part of eigenvalues (dark/light gray lines); TSR computed according with formula (31) (long dashed black line) and (32) (dashed black line); (bottom) absolute value of the two modal amplitudes.

At about time $t=13.2$, the eigenvector associated with the largest negative (fast) eigenvalue gradually rotates toward the tangential direction, becomes co-linear with it and keeps rotating away from it, as confirmed by Fig. 31 (bottom), while the corresponding eigenvalue changes from being larger (in modulus) than -1 for times $t<13.2$ to smaller (in modulus) for $t>$ 13.2: this evolution turns this fast scale into a frozen scale. In fact, as shown in Fig. 30 (bottom), the amplitude of the fastto-frozen mode is vanishingly small not because of the existence of an equilibrium but because no process is contributing to this mode. Instead the other eigenvector remains tangent to the trajectory with the corresponding eigenvalue and mode amplitude unaltered (Fig. 31 (bottom)). In this rather unusual circumstance, the TSR is still able to select, past time $\mathrm{t}=13.2$, the correct driving scale associated with the slow (and active) eigenvector tangent to the trajectory.

The effect of the combined action of one positive and one negative eigenvalue noticeable on the evolution of the circular probe drawn about the initial condition and advected by the dynamical system in Fig. 32, is a prompt elongation (caused
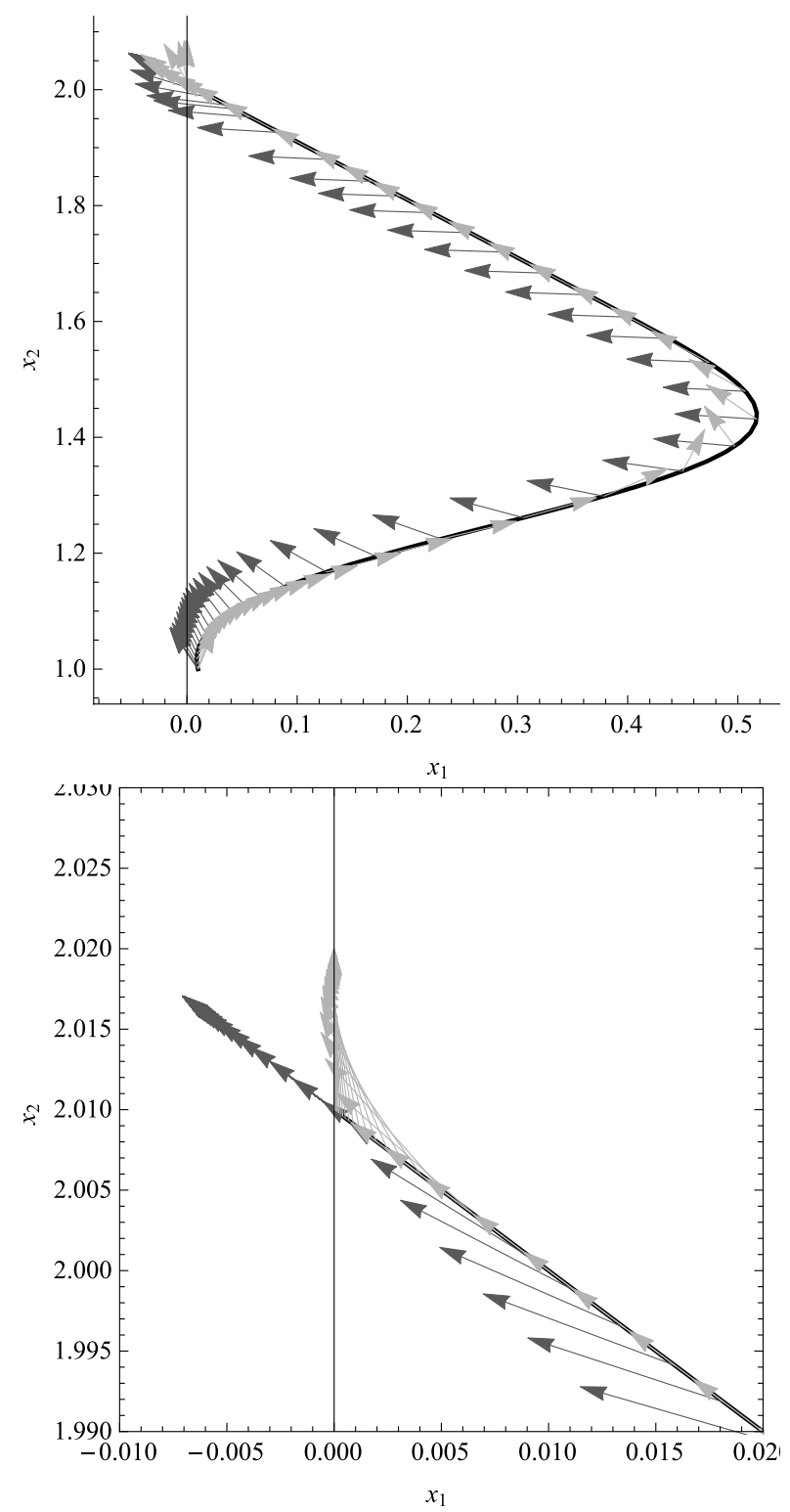

Figure 31: Evolution of eigenvectors of Williams model in phase space without crossing a region of complex eigenvalues $\left(\epsilon=0.1, \lambda_{0}=1.4, \alpha=1.0, \beta=\right.$ $\left.0.01 / \epsilon, x_{1}^{0}=\beta \epsilon=0.01, x_{2}^{0}=1.0\right)$; fast/slow eigenvectors plotted as dark/light gray arrows.

by the positive eigenvalue) of the circle along the tangential (to the trajectory) direction, while at the same time there is a strong contraction in the normal direction (caused by the negative eigenvalue).

The mechanism is so efficient that soon the circle degenerates into a one dimensional object. To make evident the action of the dynamics at later times, we created a new circular probe at the time when both eigenvalues become negative. Their action is apparent on the prompt collapse of the circle.

However, the landscape drawn by the eigenvalue fields change quite significantly with a different selection of parameters.

It suffices to set $\epsilon=0.2$ in lieu of 0.1 , for which $x_{1}^{0}=$ 


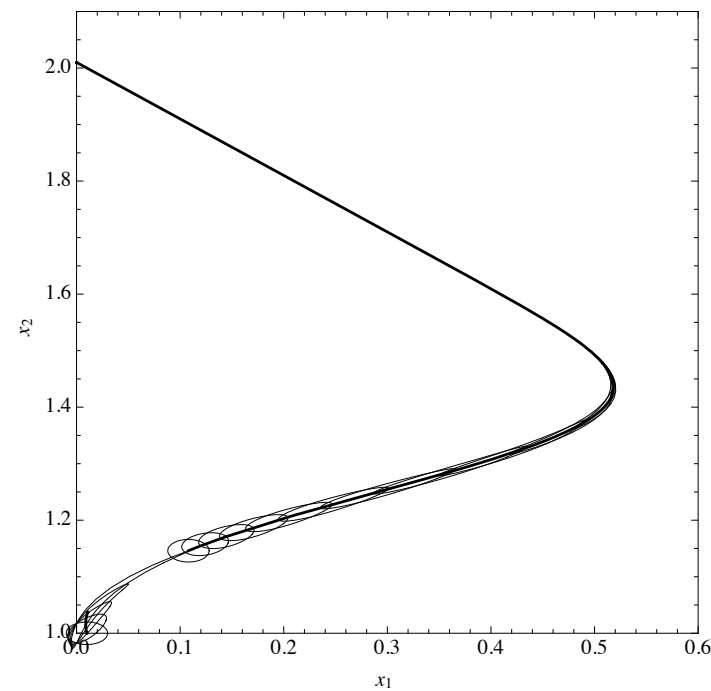

Figure 32: Evolution of the circular probe without crossing a region of complex eigenvalues $\left(\epsilon=0.1, \lambda_{0}=1.4, \alpha=1.0, \beta=0.01 / \epsilon, x_{1}^{0}=\beta \epsilon=0.01, x_{2}^{0}=1.0\right.$ ).
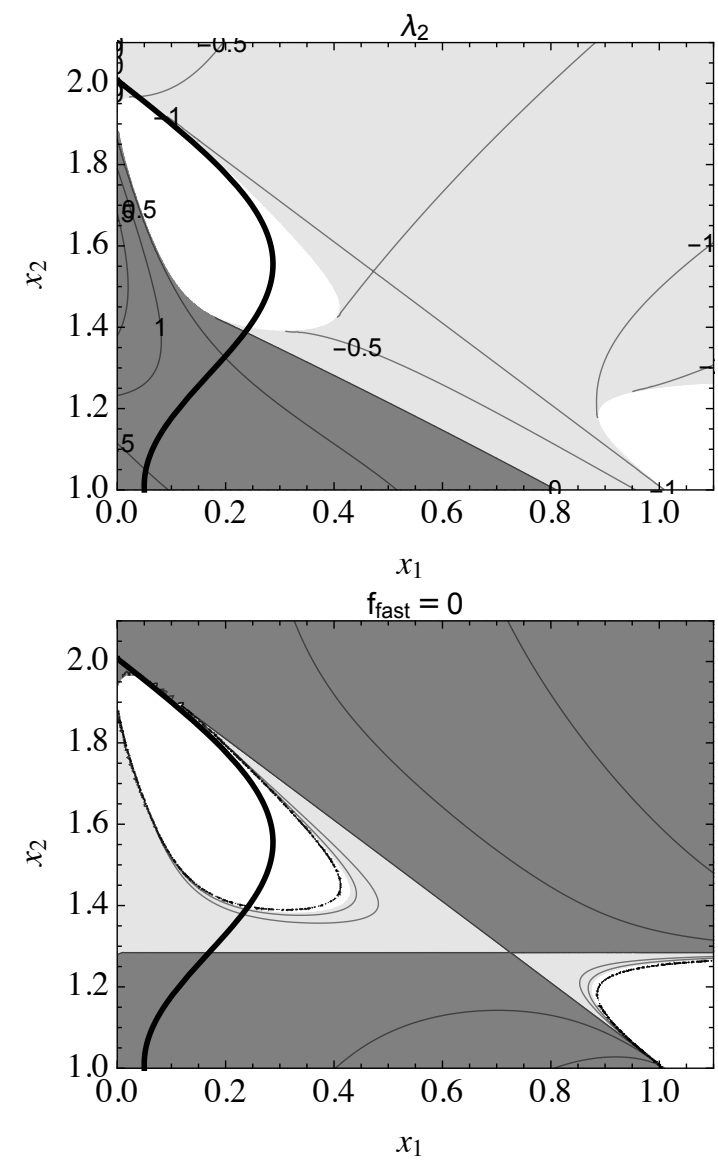

Figure 33: Evolution of the trajectory in the phase space with crossing a region of complex eigenvalues $\left(\epsilon=0.2, \lambda_{0}=1.4, \alpha=1.0, \beta=0.01 / \epsilon, x_{1}^{0}=\beta \epsilon=\right.$ $0.05, x_{2}^{0}=1.0$ ); (top) isocontour lines refer to constant fast eigenvalue magnitude; (bottom) isocontour lines refer to constant fast eigenmode magnitude.
$0.05, x_{2}^{0}=1.0$, to observe the enlargement of the upper white island and the disappearance of the lower white island (compare Fig. 29 with Fig. 33). This forces the trajectory to cross the region of complex eigenvectors. Note in Fig. 33 (bottom), that the trajectory approaches asymptotically the SIM only very late (this is so because with $\epsilon=0.2$ the system is not too stiff).

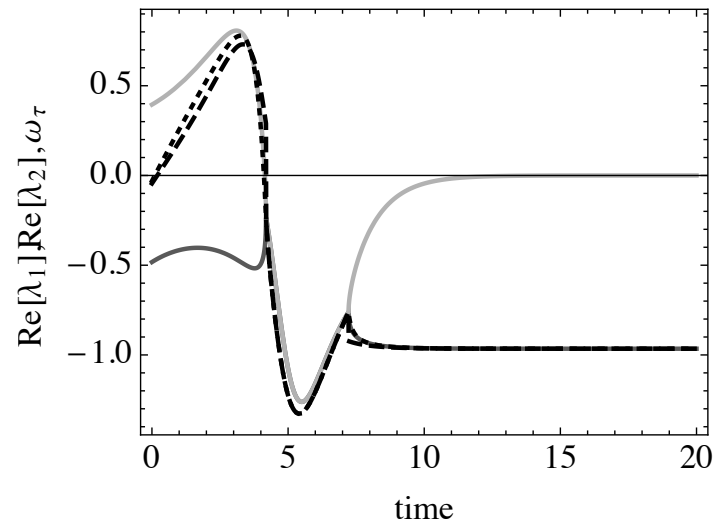

Figure 34: Time evolution of the TSR with crossing a region of complex eigenvalues $\left(\epsilon=0.2, \lambda_{0}=1.4, \alpha=1.0, \beta=0.01 / \epsilon, x_{1}^{0}=\beta \epsilon=0.05, x_{2}^{0}=1.0\right.$ ); real part of eigenvalues (dark/light gray lines); TSR computed according with formula (31) (long dashed black) and (32) (dashed black).

In Fig. 34, we can observe three interesting facts. First, the TSR is initially estimating a driving scale intermediate between the positive and negative eigenvalue; the explosive nature of the positive eigenvalue along the direction of the corresponding eigenvector is contrasted by the contractive nature of the negative eigenvalue along the direction of the corresponding eigenvector, this resulting in a less explosive driving scale along the tangential direction.

Note that none of both eigenvectors is initially aligned with the trajectory (Fig. 35), which instead starts along a direction intermediate between the two eigenvectors; second, the real part of the complex pair of eigenvalues is always negative; third, the TSR marks the fastest mode as driving for the same reason discussed earlier, that is the slowest mode is frozen, and the fixed point is approached at the pace set by the slow active scale tracked by the TSR.

\section{TSR Analysis of Hydocarbon Fuels Ignition}

We are now ready to apply Eqs. (30)-(31) with the weight definition of (32), to the branched-chain/thermal, adiabatic isochoric auto-ignition processes of methane/air, propane/air and $\mathrm{n}$-heptane/air oxidation mechanisms. The set of ordinary differential equations that describes the time evolution of a spatially homogeneous constant volume batch reactor is well documented in many text books and thus we will not report it here.

We adopted the formulation described in the CHEMKIN manual [15]; however, the management of all the thermokinetic datasets has been carried out by resorting to TChem [16]. 

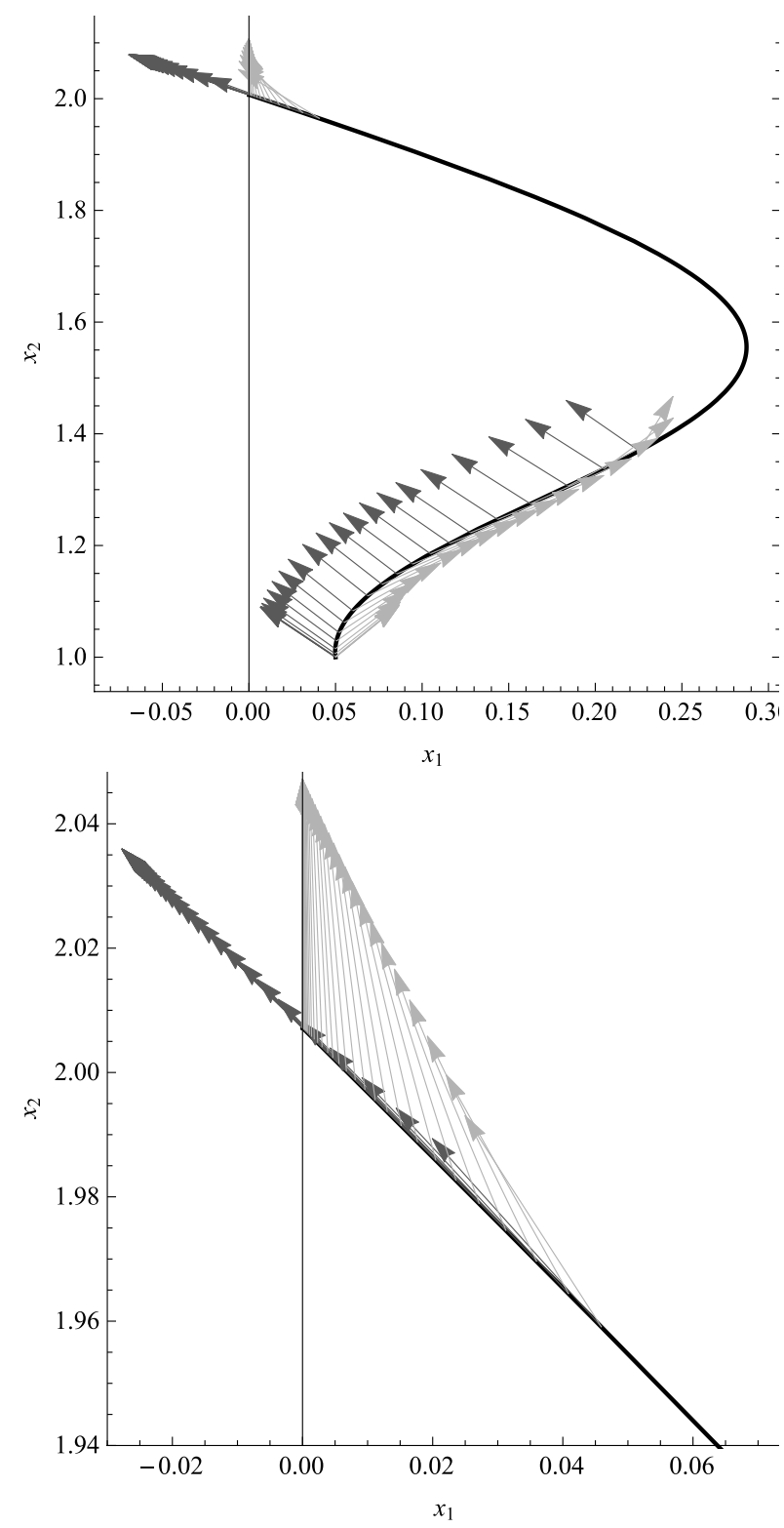

Figure 35: Evolution of eigenvectors of Williams model in phase space ( $\epsilon=$ $\left.0.2, \lambda_{0}=1.4, \alpha=1.0, \beta=0.01 / \epsilon, x_{1}^{0}=\beta \epsilon=0.05, x_{2}^{0}=1.0\right)$; fast $/$ slow eigenvectors plotted as dark/light gray arrows.

The CSP and TSR analyses are carried out by resorting to $\mathrm{CSPTK}^{3}$.

The numerical solution is carried out using DVODE [17].

We adopt the GRI 3.0 mechanism for methane [18], and the Curran mechanisms [19], for the other two fuels.

The initial conditions for all three fuels are set at $p_{0}=1 \mathrm{~atm}$, $T_{0}=1000 \mathrm{~K}$, and the mixture composition is stoichiometric.

\footnotetext{
${ }^{3}$ CSPTk - A Software Toolkit for the CSP and TSR Analysis of Kinetic Models and the Simplification and Reduction of Chemical Kinetics Mechanisms), 2015. The software can be obtained upon request from M.Valorani (mauro.valorani@uniroma1.it).
}

\subsection{Methane Ignition}

In Fig. 36, we plot the real parts of the negative/positive eigenvalues in light-gray/black markers, respectively, for the methane mechanism. The negative eigenvalues are plotted using the absolute value of the real parts to plot them on the logscale. In the following, the eigenvalues are ordered according to increasing magnitude of their modulus.

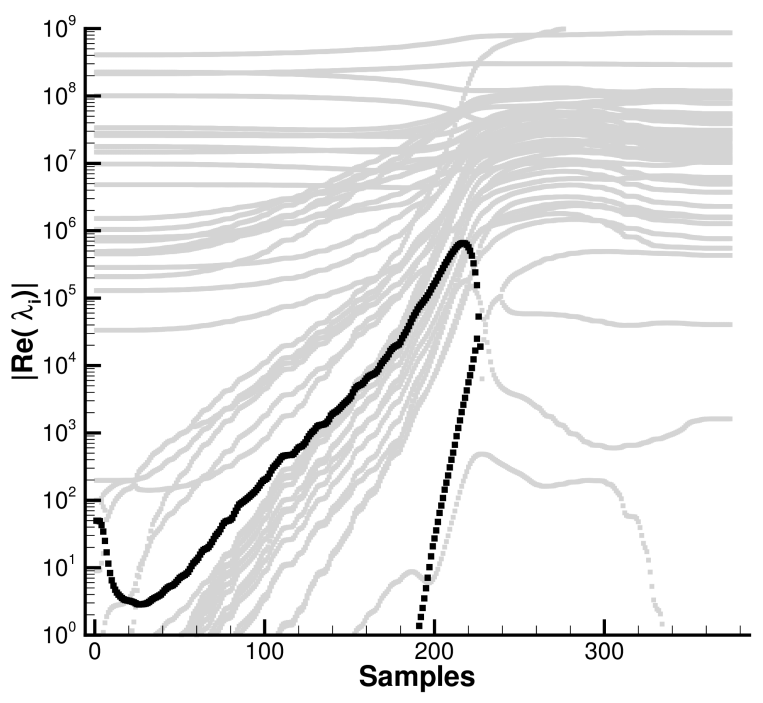

Figure 36: Methane Gri 3.0 mechanism: a pair of positive eigenvalues merge similarly to what observed in Williams model; absolute values of eigenvalues with positive real parts (black markers), and negative real parts (light-gray markers). Samples are taken in the time interval $t=[0-1.08] \mathrm{s}$.

The black markers clearly show that there exists two eigenvalue with positive real part. At about sample \#250, the two eigenvalues merge to form a complex conjugate pair with positive real parts similarly to what observed in the Williams' model.

Still later, the complex conjugate pair crosses the imaginary axis so that the real parts become negative. Next, the imaginary parts reduce to zero, and several real negative eigenvalues emerge and remain until the system reaches the equilibrium state. In contrast to what happens in Williams' model, it is now difficult to identify the pair of negative eigenvalues emerging from the coalescence of the two positive ones. We will see shortly that this identification can be readily carried out by resorting to the TSR.

However, the qualitative similarity of the behavior between Williams' model and the real kinetics associated with the presence and the dynamics of the pair of positive eigenvalues can be considered at this stage ascertained.

The tangential stretch rate $\bar{\omega}_{\tilde{\tau}}$ (computed without considering the direction cosine in the weights, formula (32)) for the methane mechanism is shown in Fig. 37 together with the modes mostly contributing to the active subspace. This subspace is spanned by all the modes which have a participation in$\operatorname{dex} P_{\text {mode }_{i}}^{\bar{\omega}_{\tilde{\tau}}}>10^{-2}$. Red/Yellow markers indicates when $\omega_{t}$ has 
a positive/negative sign, this denoting an explosive/dissipative stage).

Inspection of Fig. 37 reveals that $\bar{\omega}_{\tilde{\tau}}$, after a short initial transient, becomes coincident with $\lambda_{a+}$. A little earlier than the merging of the two positive eigenvalue, $\bar{\omega}_{\tilde{\tau}}$ departs from $\lambda_{a+}$ for two reasons.

Firstly, in Fig. 38 we draw a marker for all positive eigenvalues having a participation index $P_{\text {mode }_{i}}^{\bar{\omega}_{\tilde{\tau}}}>10^{-2}$. It is apparent that the slow positive eigenvalue becomes important only earlier than its merging with the largest positive eigenvalue for all the three fuels considered.

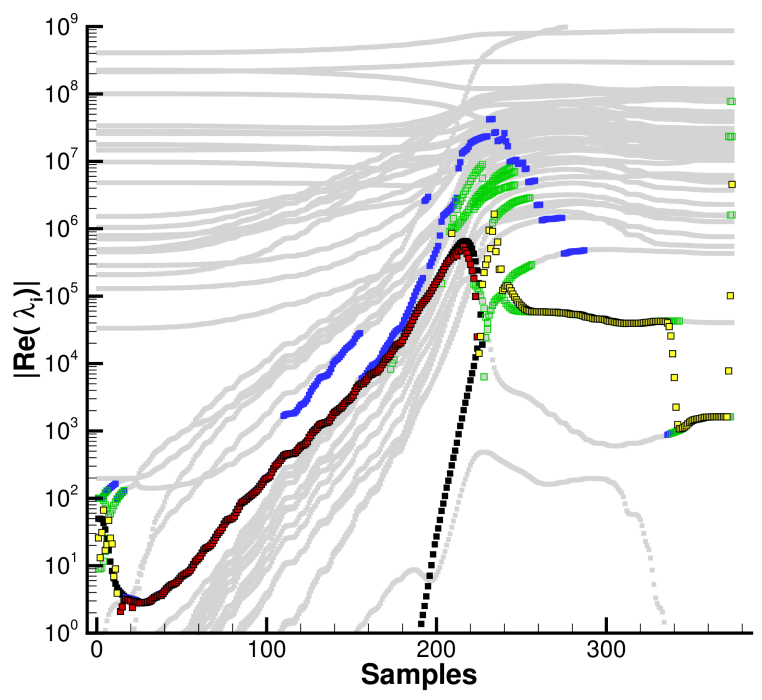

Figure 37: TSR analysis for methane: absolute values of eigenvalues with negative real parts (light-gray markers), fastest of the slow CSP modes $\lambda_{M+1}$ (blue markers), $\omega_{t}$ with positive sign (red markers), $\omega_{t}$ with negative sign (yellow markers), and active subspace (green markers) computed without considering the direction cosine in the weights (formula (32).

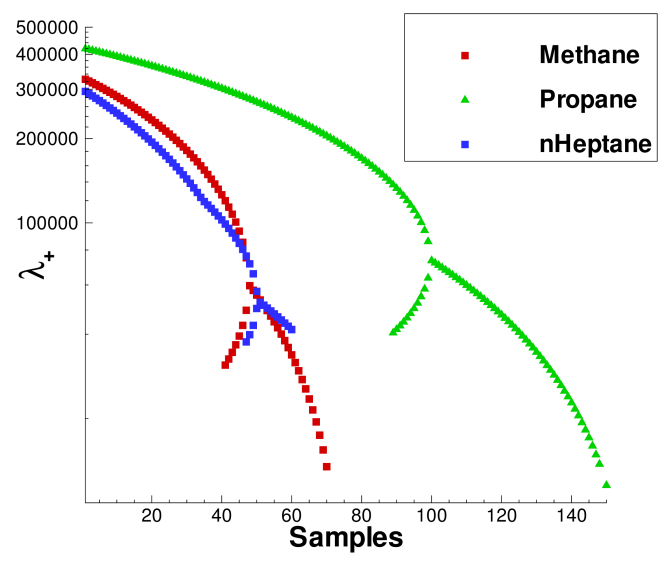

Figure 38: Positive eigenvalues $\left(\lambda_{+}\right)$with $P_{\lambda_{+}}^{\bar{\omega}_{\tilde{\tau}}}>10^{-2}$ for methane, propane, and n-heptane.
Secondly, the contribution of dissipative scales (green markers) to the positive $\bar{\omega}_{\tilde{\tau}}$ (red markers) increases just near the merging, with the effect of reducing the magnitude of the positive $\bar{\omega}_{\tilde{\tau}}$ (the red markers are drawn somewhat lower than the black markers associated with the largest positive eigenvalue), whereas the negative $\bar{\omega}_{\tilde{\tau}}$ (yellow markers) first tracks the fastest negative eigenvalue (green markers) of the pair emanating after the merging of the two positive eigenvalues, and later $\bar{\omega}_{\tilde{\tau}}$ smoothly transitions and becomes coincident with the slowest negative eigenvalue of the pair, while the system progresses towards equilibrium.

It is worth noticing how the fastest of the slow CSP modes $\lambda_{M+1}$ (blue markers) defines the upper boundary of the dissipative scales (green markers) contributing to $\bar{\omega}_{\tilde{\tau}}$. We recall that according to CSP, all scales larger than $\lambda_{M}$ lie in the fast subspace, and all lower than $\lambda_{M+1}$ lie in the slow subspace.

As anticipated earlier, the participation index to TSR, $P_{i}^{\bar{\omega}_{\tilde{\tau}}}$, allow us to identify the pair of eigenvalues with negative real part associated with the pair of eigenvalues with positive real part, so as to complete the analogy with the Williams' model. All these events are clearly noticeable in Fig. 39.

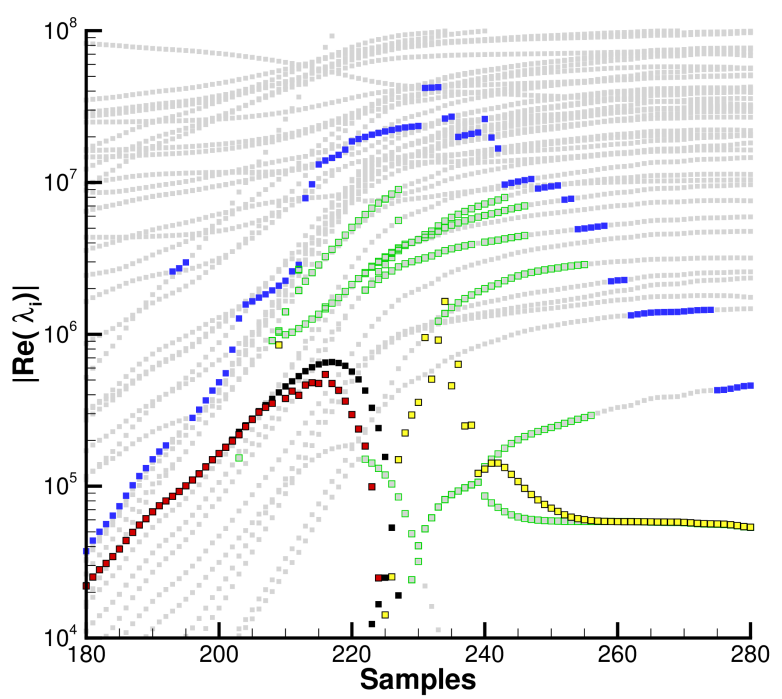

Figure 39: Enlargement of Fig. 37: the fastest of the slow CSP modes $\lambda_{M+1}$ (blue markers) defines the upper boundary of the dissipative scales (green markers) contributing to $\bar{\omega}_{\tilde{\tau}}$ (red/yellow markers for positive/negative TSR, respectively); absolute values of eigenvalues with positive real parts (black markers), absolute values of eigenvalues with negative real parts (light-gray markers).

It is of interest to identify what are the reactions contributing the most to the TSR during the different stages of the ignition process. To this aim, we use the index $P_{\text {reaction }_{k}}^{\bar{\omega}_{\tilde{\tau}}}$, as defined in (37). It is also of interest to identify what are the species involved in the most important reactions. This investigation can be readily carried out by first sorting out the important modes to the TSR as those having a sufficiently large participation index to TSR, $P_{\text {mode }}^{\bar{\omega}_{\tilde{\tau}}}$, and then to find the CSP pointed species associated with the "important-to-TSR modes". The outcome 


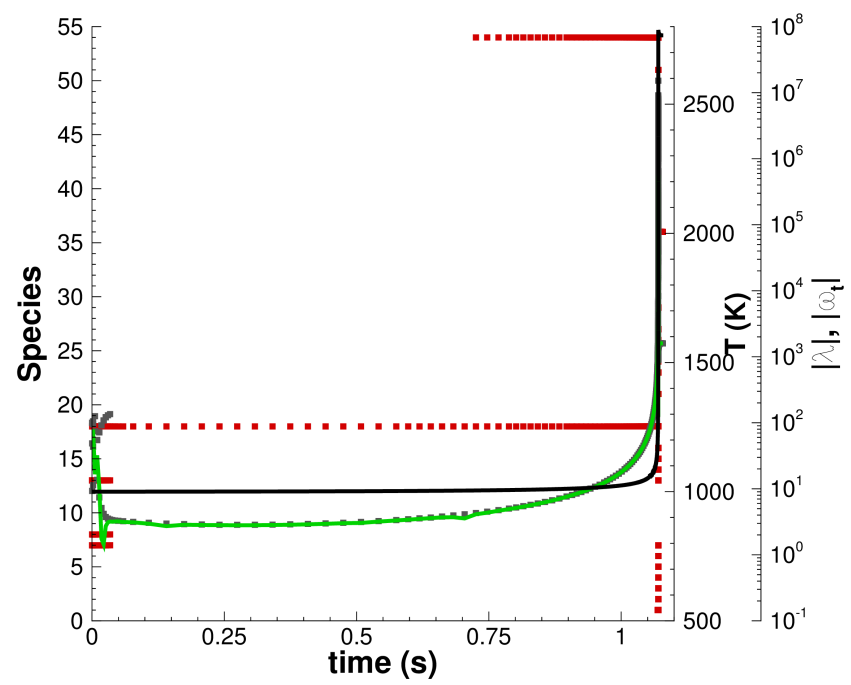

Figure 40: Most important species during the explosion regime of methane/air ignition; Red markers: CSP pointed species (\# on left Y axis), Black solid line: Temperature (\# on right $Y$ axis), Green solid line: $\left|\bar{\omega}_{\tilde{\tau}}\right|$ (\# on second Y right axis), Grey markers: $|\operatorname{Re}(\operatorname{active} \lambda)|$ (\# on second Y right axis).

of this exercise is reported in Fig. 40, which indicates that until time $\mathrm{t}=0.05$, there are 4 species marked as important, that is species \#7, 8, 13, and 18, which in GRI 3.0 correspond to $\mathrm{HO}_{2}, \mathrm{H}_{2} \mathrm{O}_{2}, \mathrm{CH}_{2}$, and $\mathrm{CH}_{2} \mathrm{O}$, respectively; the most important reactions involving these species are listed in Table 1.

\begin{tabular}{ccc} 
Reaction \# & $P_{\text {reaction }}^{\bar{\omega}_{\tilde{\tau}}}$ & Reaction \\
\hline R\#444 & $+9.40 \mathrm{E}-01$ & $\mathrm{HO}_{2}+\mathrm{CH}_{3} \leftarrow \mathrm{O}_{2}+\mathrm{CH}_{4}$ \\
R\#483 & $+2.78 \mathrm{E}-02$ & $\mathrm{CH}_{3}+\mathrm{H}_{2} \mathrm{O}_{2} \leftarrow \mathrm{HO}_{2}+\mathrm{CH}_{4}$ \\
R\#156 & $+2.09 \mathrm{E}-02$ & $\mathrm{CH}_{3}+\mathrm{O}_{2} \rightarrow \mathrm{O}+\mathrm{CH}_{3} \mathrm{O}$ \\
R\#157 & $+1.49 \mathrm{E}-02$ & $\mathrm{CH}_{3}+\mathrm{O}_{2} \rightarrow \mathrm{OH}+\mathrm{CH}_{2} \mathrm{O}$
\end{tabular}

Table 1: Initiation: $\mathrm{t}=1.11786775 \mathrm{E}-03$

The species $\mathrm{CH}_{2} \mathrm{O}$ (\#18 in Fig. 40)) is persistently marked as important throughout the whole incubation period. The reactions important in the incubation period, clearly also involving $\mathrm{CH}_{2} \mathrm{O}$, are listed in Table 2.

\begin{tabular}{ccc} 
Reaction \# & $P_{\text {reaction }_{k}}^{\bar{\omega}_{\tilde{k}}}$ & Reaction \\
\hline R\#157 & $+1.83 \mathrm{E}-01$ & $\mathrm{CH}_{3}+\mathrm{O}_{2} \rightarrow \mathrm{OH}+\mathrm{CH}_{2} \mathrm{O}$ \\
R\# 32 & $+1.43 \mathrm{E}-01$ & $\mathrm{O}_{2}+\mathrm{CH}_{2} \mathrm{O} \rightarrow \mathrm{HO}_{2}+\mathrm{HCO}$ \\
R\#159 & $-1.70 \mathrm{E}-01$ & $2 \mathrm{CH}_{3}(+\mathrm{M}) \rightarrow \mathrm{C}_{2} \mathrm{H}_{6}(+\mathrm{M})$
\end{tabular}

Table 2: Incubation Period: $\mathrm{t}=3.94445859 \mathrm{E}-01$

Temperature (\#54 in Fig. 40) is marked as important only past time $t=0.73$. Indeed, it is well known that the incubation period is eminently isothermal.

The list of important (pointed species) during the explosion regime of methane/air ignition is somewhat wider than the one

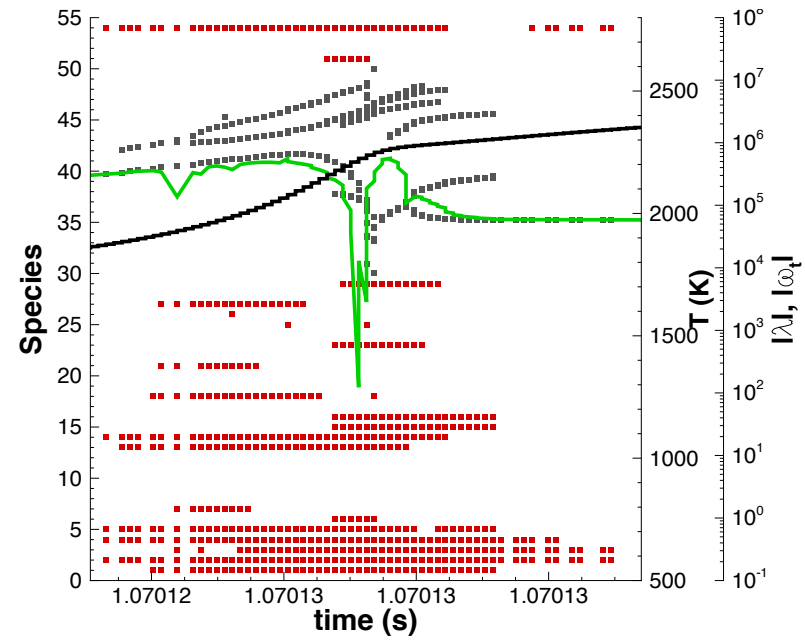

Figure 41: Most important species during the explosion regime of methane/air ignition; Red markers: CSP pointed species (\# on left Y axis), Black solid line: Temperature (\# on right $\mathrm{Y}$ axis), Green solid line: $\left|\bar{\omega}_{\tilde{\tau}}\right|$ (\# on second Y right axis), Grey markers: $\mid \operatorname{Re}($ active $\lambda) \mid$ (\# on second $\mathrm{Y}$ right axis).

during the initiation regime. The explosion regime occurs at about time $\mathrm{t}=1.0701 \mathrm{~s}$ just when $\bar{\omega}_{\tilde{\tau}}$ attains its peak value. Inspection of Fig. 41 shows that the list includes: \#1 $-\mathrm{H}_{2}$, \#2 - $\mathrm{H}, \# 3-\mathrm{O}, \# 4-\mathrm{O}_{2}, \# 5-\mathrm{OH}, \# 13-\mathrm{CH}_{3}, \# 14-\mathrm{CH}_{4}, \# 18-$ $\mathrm{CH}_{2} \mathrm{O}$, \#25 $-\mathrm{C}_{2} \mathrm{H}_{4}, \# 27-\mathrm{C}_{2} \mathrm{H}_{6}$, and temperature. The reactions marked as important in the explosion regime of methane are listed in Table 3, where it is also indicated the mode number associated with the pointed reaction, and the real part of the corresponding eigenvalue. Note that during the explosion, there are four important modes, namely mode \#15, \#19, \#20 and \#22, the first being associated with the eigenvalue with a positive real part, $\lambda_{+}=+6.5 \mathrm{E}+05$, and the others with eigenvalues with negative real parts, $\lambda_{-}=-1.44 \mathrm{E}+06,-1.47 \mathrm{E}+06$, and $-3.19 \mathrm{E}+06$, respectively. Under those circumstances, $\bar{\omega}_{\tilde{\tau}}=5.6 E+05$, that is slightly lower than the positive eigenvalue. The reactions

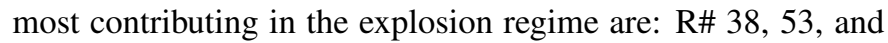
99, that is reactions $\mathrm{H}+\mathrm{O}_{2} \rightarrow \mathrm{O}+\mathrm{OH}, \mathrm{CH}_{4}+\mathrm{H} \rightarrow \mathrm{CH}_{3}+\mathrm{H} 2$ and $\mathrm{OH}+\mathrm{CH}_{4} \rightarrow \mathrm{CH}_{3}+\mathrm{H}_{2} \mathrm{O}$, respectively. Note that the first reaction is the most contributing to the positive eigenvalue $\lambda_{+}$.

\begin{tabular}{ccccc}
$\#$ & $\mathrm{Re}[\lambda]$ & $\mathrm{R} \#$ & $P_{\text {reaction }_{k}}^{\bar{\omega}_{\tilde{\tau}}}$ & Reaction \\
\hline 15 & $6.50 \mathrm{E}+05$ & 38 & $2.88 \mathrm{E}-01$ & $\mathrm{H}+\mathrm{O}_{2} \rightarrow \mathrm{O}+\mathrm{OH}$ \\
19 & $-1.44 \mathrm{E}+06$ & 38 & $-1.68 \mathrm{E}-01$ & $\mathrm{H}+\mathrm{O}_{2} \rightarrow \mathrm{O}+\mathrm{OH}$ \\
19 & $-1.44 \mathrm{E}+06$ & 53 & $1.39 \mathrm{E}-01$ & $\mathrm{H}+\mathrm{CH}_{4} \rightarrow \mathrm{CH}_{3}+\mathrm{H} 2$ \\
20 & $-1.47 \mathrm{E}+06$ & 38 & $1.35 \mathrm{E}-01$ & $\mathrm{H}+\mathrm{O}_{2} \rightarrow \mathrm{O}+\mathrm{OH}$ \\
20 & $-1.47 \mathrm{E}+06$ & 53 & $-1.66 \mathrm{E}-01$ & $\mathrm{H}+\mathrm{CH}_{4} \rightarrow \mathrm{CH}_{3}+\mathrm{H} 2$ \\
22 & $-3.19 \mathrm{E}+06$ & 38 & $1.11 \mathrm{E}-01$ & $\mathrm{H}+\mathrm{O}_{2} \rightarrow \mathrm{O}+\mathrm{OH}$ \\
22 & $-3.19 \mathrm{E}+06$ & 53 & $-1.61 \mathrm{E}-01$ & $\mathrm{H}+\mathrm{CH}_{4} \rightarrow \mathrm{CH}_{3}+\mathrm{H} 2$ \\
22 & $-3.19 \mathrm{E}+06$ & 99 & $-1.06 \mathrm{E}-01$ & $\mathrm{OH}+\mathrm{CH}_{4} \rightarrow \mathrm{CH}_{3}+\mathrm{H}_{2} \mathrm{O}$
\end{tabular}

Table 3: Explosion period: $\mathrm{t}=1.07 \mathrm{~s}, \bar{\omega}_{\tilde{\tau}}=5.6 E+05$.

Here, we want to stress again that the process of combining the important modes with the CSP pointers is made fully auto- 
matic thanks to the Participation Index of a mode to the TSR, $P_{\text {mode }}^{\bar{\omega}_{\bar{\tau}}}$, which allows us to identify the modes most contributing to the most energy containing scale. In the standard CSP method, the CSP pointers are used to identify the minor species related to the fast subspace, and therefore the mode selection is obviously made by picking all modes spanning the fast subspace. However, as discussed in this paper, not all the modes spanning the slow subspace equally contribute to the most energy containing kinetics. This is where the TSR concept comes very handy because it allows an automatic identification of the modes most important in the slow subspace.

\subsection{Propane, and n-Heptane Ignition}

We carry out the analysis for two other fuels: propane and nheptane. Figure 42 compares the behavior of the positive eigenvalues and of the largest (absolute value) negative eigenvalues for the three fuels. It can be noted that the qualitative behavior is very similar, independently of the complexity of the mechanism. Furthermore, the peak values of the positive eigenvalues are very similar: $6.56 \times 10^{5}, 7.50 \times 10^{5}$ and $7.13 \times 10^{5}$ for methane, propane and n-heptane, respectively.

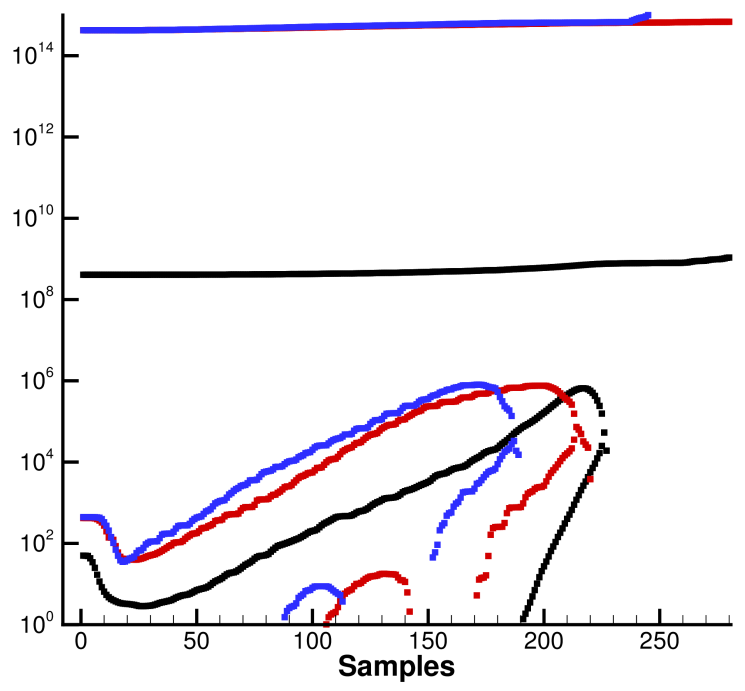

Figure 42: Comparing eigenvalues with positive real part (range $10^{0}-10^{6}$ ) and fastest eigenvalues with negative real part (range $10^{9}-10^{15}$ ) during ignition of methane (black), propane (red), and n-heptane (blue).

The tangential stretch rate $\bar{\omega}_{\tilde{\tau}}$ for the propane and n-heptane mechanisms is shown in Figs. 43 and 44 together with the eigenvalues of the modes mostly contributing to the active subspace (modes having a participation index $P_{\text {mode }_{i}}^{\bar{\omega}_{\tilde{\tau}}}>10^{-2}$ ). Comparing Figs. 37, 43, and 44 reveals that $\bar{\omega}_{\tilde{\tau}}$, after a short initial transient, becomes also coincident with $\lambda_{a+}$. Therefore, $\bar{\omega}_{\tilde{\tau}}$ properly identifies which positive eigenvalue is the controlling one if more than one is simultaneously present.

It is worth noting that methane, propane and n-heptane attain nearly the same maximum positive value of $\bar{\omega}_{\tilde{\tau}}: 5.6 \times 10^{5}, 6.5 \times$ $10^{5}$ and $6.4 \times 10^{5}$, respectively. Besides the region about the

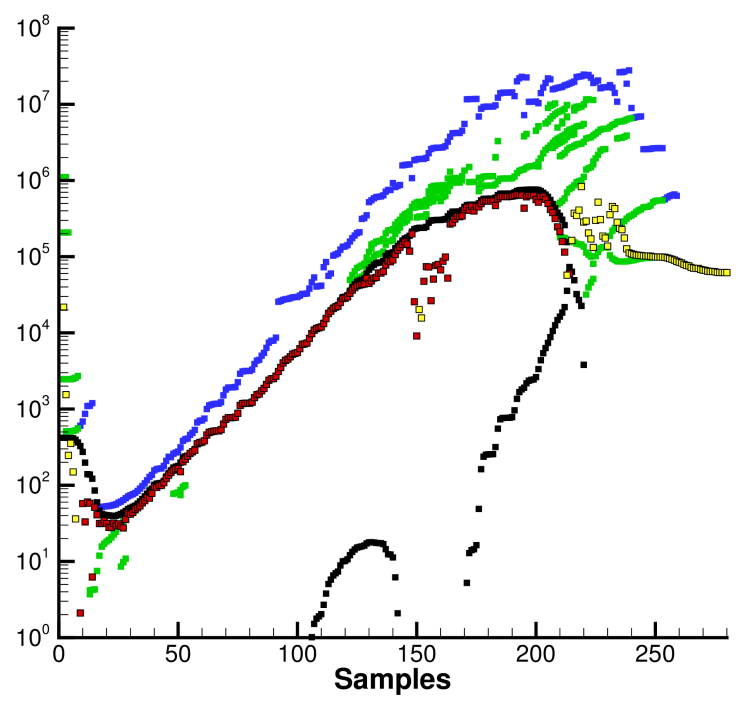

Figure 43: TSR analysis for propane: absolute values of eigenvalues with positive real parts (black markers), fastest of the slow CSP modes $\lambda_{M+1}$ (blue markers), $\omega_{t}$ with positive sign (red markers), $\omega_{t}$ with negative sign (yellow markers), and active subspace (green markers) computed without considering the direction cosine in the weights (formula (32).

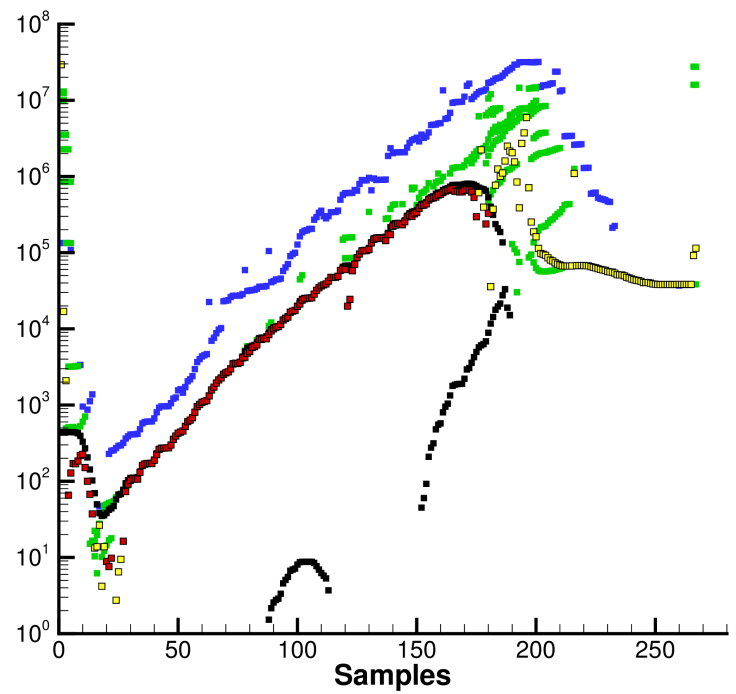

Figure 44: TSR analysis for n-heptane: absolute values of eigenvalues with positive real parts (black markers), fastest of the slow CSP modes $\lambda_{M+1}$ (blue markers), $\omega_{t}$ with positive sign (red markers), $\omega_{t}$ with negative sign (yellow markers), and active subspace (green markers) computed without considering the direction cosine in the weights (formula (32).

eigenvalues merging, very few modes contribute to $\bar{\omega}_{\tilde{\tau}}$, that is, the active scale range is dominated by very few modes for most of the ignition process.

It is interesting to compare the time evolution of $\lambda_{M+1}$, the fastest of the slow modes according with the CSP method, 
where the number of exhausted modes $\mathrm{M}$ is found by applying the CSP criterion (25). In Figs. 37-44, it is possible to observe that $\lambda_{M+1}$ is much faster than (i) the most energetic scale (indicated by $\bar{\omega}_{\tilde{\tau}}$ ), and (ii) the fastest mode of the active subspace.

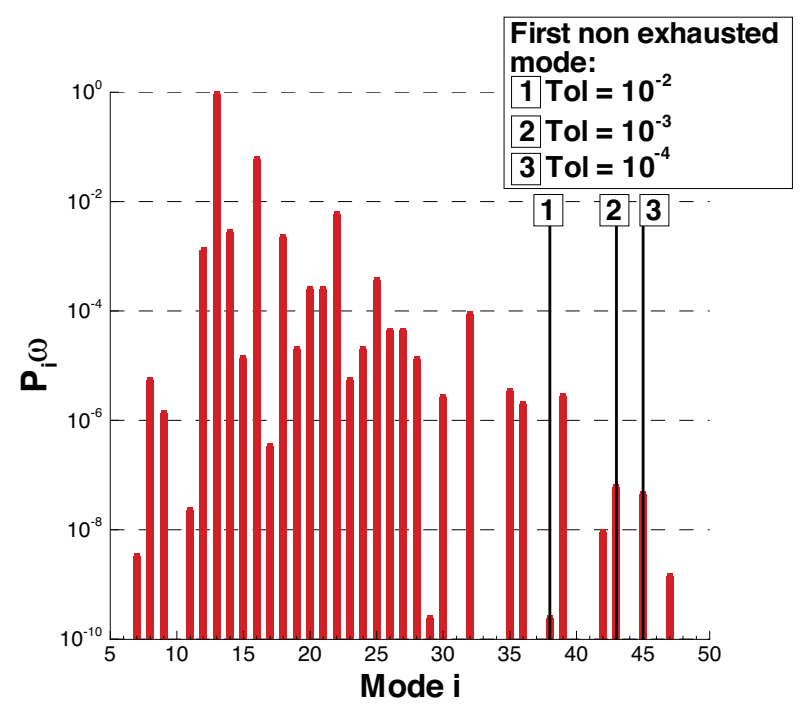

Figure 45: Propane participation indices and first non-exhausted mode for different tolerances, sample \#170 (see Fig. 43).

Figure 45 shows the participation indices $P_{\text {mode }}^{\bar{\omega}_{\tilde{\tau}}}$ for the propane mechanism at sample \#170, which corresponds to the instant when $\bar{\omega}_{\tilde{\tau}}$ has its maximum value during the explosion. It can be seen that only two modes, one characterized by an eigenvalue with negative real part (mode \#16) and the other one by an eigenvalue with positive real part (mode \#13), are above the selected tolerance of $10^{-2}$.

By construction, the number of CSP exhausted modes $M$ is a function of the user defined error threshold. Indeed, we found at sample \#170, that $M=39,44,46$ when rtol in Eq. (25) is set to $10^{-2}, 10^{-3}$, and $10^{-4}$, respectively. The figure shows that, for all the error thresholds, the modes just slower than the first non-exhausted mode, $M+1$, do not contribute much to $\bar{\omega}_{\tilde{\tau}}$, all having a $P_{\text {mode }}^{\bar{\omega}_{\tilde{\tau}}}$ below $10^{-6}$, and that the amplitude of these modes are well below the threshold chosen for the computation of the participation index $\left(10^{-2}\right)$.

Figure 46 shows the participation index of the $k-$ th reaction to the i-th mode having eigenvalues with positive real parts, $P_{\text {reaction }_{k}}^{\text {mode }_{i}}(\lambda+)$, together with the participation index $P_{\text {reaction }_{k}}^{\text {mode }_{i}}\left(\bar{\omega}_{\tilde{\tau}}\right)$, for the case of propane, still at sample \#170. As noted above, only few modes contribute to the range of active scales and, consequently, the most significant reactions to the active dynamics are those contributing to these few modes (all reactions with a large CSP Participation index to the important modes for $\bar{\omega}_{\tilde{\tau}}$ ), as already discussed in [23]. Note from Figs. 45 and 46 that, at this sample, the slow positive eigenvalue (mode \#7) and consequently the reactions important for it, does not participate to $\bar{\omega}_{\tilde{\tau}}$, while a negative one (mode \#16) does participate.

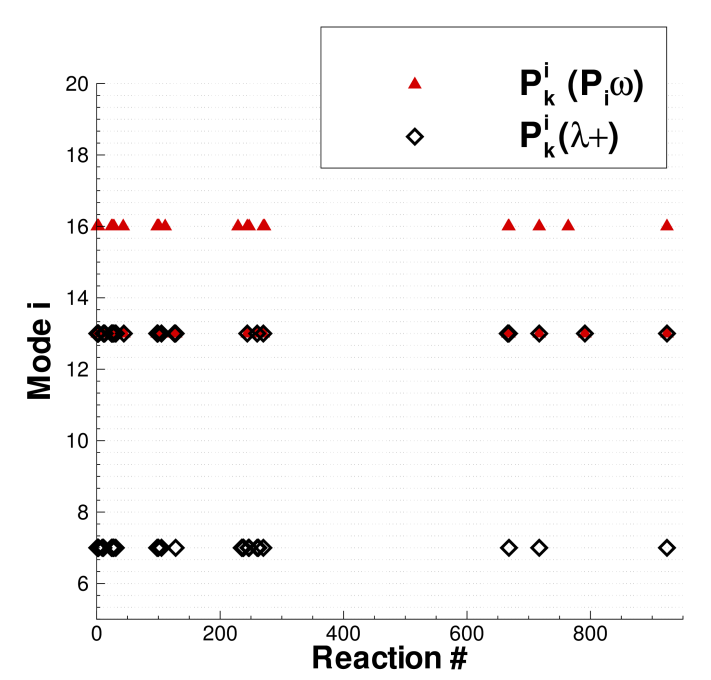

Figure 46: Propane reaction indices for sample \#170 (see Fig. 43).

At this stage, it is interesting to identify all the reactions that are important to $\bar{\omega}_{\tilde{\tau}}$ and that are common to all fuels. At the selected samples, the important modes for the TSR are three, one characterized by an eigenvalue with a positive real part, and the other two by eigenvalues with negative real parts. We focus our attention to the instant at which the TSR has its maximum value during the ignition event (Table 4).

\begin{tabular}{ccc} 
Fuel & Sample \# & Time $(\mathrm{s})$ \\
\hline methane & $\# 227$ & $1.0701 \times 10^{0}$ \\
propane & $\# 170$ & $5.0994 \times 10^{-2}$ \\
n-heptane & $\# 151$ & $5.25 \times 10^{-2}$
\end{tabular}

Table 4: Time instants at which TSR attains its maximum value during the ignition event of the 3 fuels.

The results discussed in the following are not specific to the particular sample chosen: they persist over a rather large interval of samples, centered at the point of maximum $\bar{\omega}_{\tilde{\tau}}$.

The reactions important for the eigenvalue with positive/negative real part are reported in Table 5/6, respectively. The two most important reactions for the eigenvalue with positive real part are $\mathrm{H}_{2}-\mathrm{O}_{2}$ chain reactions: $\mathrm{H}+\mathrm{O}_{2} \rightarrow \mathrm{O}+\mathrm{OH}$ and $\mathrm{OH}+\mathrm{H}_{2} \rightarrow \mathrm{H}+\mathrm{H}_{2} \mathrm{O}$. The third is a chain-branching reaction involving the consumption of $\mathrm{HCO}$ to form $\mathrm{H}$ and $\mathrm{CO}$. In the fourth reaction, the radical $\mathrm{CH}_{2}$ (s) reacts with $\mathrm{O}_{2}$ to form the highly active $\mathrm{H}$ and $\mathrm{OH}$ radicals (chain-branching), speeding up the overall oxidation. The next reaction is the dominant path for $\mathrm{CO}$ oxidation $\mathrm{CO}+\mathrm{OH} \rightarrow \mathrm{CO}_{2}+\mathrm{H}$, which also generates the $\mathrm{H}$ radicals needed by the first reaction.

Considering the modes with eigenvalues with negative real parts, the two most important reactions are $\mathrm{CH}_{4}+\mathrm{OH} \rightarrow \mathrm{CH}_{3}$ $+\mathrm{H}_{2} \mathrm{O}$, that is the methane consumption achieved through $\mathrm{H}-$ abstraction by $\mathrm{OH}$ and producing a methyl radical. The second reaction is the methyl radical consumption by $\mathrm{O}$ atoms through $\mathrm{CH}_{3}+\mathrm{O} \rightarrow \mathrm{CH}_{2} \mathrm{O}+\mathrm{H}$. 


\begin{tabular}{|c|c|c|}
\hline Reaction & $\left|P_{\text {reaction }_{k}}^{\text {mode }_{i}}\right|$ & $\left|P_{\text {reaction }} \bar{\omega}_{\tilde{\tau}}\right|$ \\
\hline $\mathrm{H}+\mathrm{O}_{2} \rightarrow \mathrm{O}+\mathrm{OH}$ & $0.2234 \times 10^{-0}$ & $0.2159 \times 10^{-0}$ \\
\hline $\mathrm{OH}+\mathrm{H}_{2} \rightarrow \mathrm{H}+\mathrm{H}_{2} \mathrm{O}$ & $0.8346 \times 10^{-1}$ & $0.8067 \times 10^{-1}$ \\
\hline $\mathrm{HCO}+\mathrm{M} \rightarrow \mathrm{H}+\mathrm{CO}+\mathrm{M}$ & $0.6036 \times 10^{-1}$ & $0.5834 \times 10^{-1}$ \\
\hline $\mathrm{CH}_{2}(\mathrm{~s})+\mathrm{O}_{2} \rightarrow \mathrm{CO}+\mathrm{OH}+\mathrm{H}$ & $0.4243 \times 10^{-1}$ & $0.4101 \times 10^{-1}$ \\
\hline $\mathrm{CO}+\mathrm{OH} \rightarrow \mathrm{CO}_{2}+\mathrm{H}$ & $0.3240 \times 10^{-1}$ & $0.3131 \times 10^{-1}$ \\
\hline $\mathrm{O}+\mathrm{H}_{2} \rightarrow \mathrm{H}+\mathrm{OH}$ & $0.2795 \times 10^{-1}$ & $0.2702 \times 10^{-1}$ \\
\hline $\mathrm{OH}+\mathrm{H}_{2} \leftarrow \mathrm{H}+\mathrm{H}_{2} \mathrm{O}$ & $0.2531 \times 10^{-1}$ & $0.2446 \times 10^{-1}$ \\
\hline $\mathrm{CH}_{2}(\mathrm{~s})+\mathrm{M} \rightarrow \mathrm{CH}_{2}+\mathrm{M}$ & $0.2347 \times 10^{-1}$ & $0.2268 \times 10^{-1}$ \\
\hline $\mathrm{CH}_{3}+\mathrm{OH} \rightarrow \mathrm{CH}_{2}(\mathrm{~s})+\mathrm{H}_{2} \mathrm{O}$ & $0.2268 \times 10^{-1}$ & $0.2192 \times 10^{-1}$ \\
\hline $\mathrm{CH}_{4}+\mathrm{H} \rightarrow \mathrm{CH}_{3}+\mathrm{H}_{2}$ & $0.2091 \times 10^{-1}$ & $0.2021 \times 10^{-1}$ \\
\hline $\mathrm{C}_{2} \mathrm{H}_{4}+\mathrm{H} \rightarrow \mathrm{C}_{2} \mathrm{H}_{3}+\mathrm{H}_{2}$ & $0.1901 \times 10^{-1}$ & $0.1838 \times 10^{-1}$ \\
\hline $\mathrm{H}+\mathrm{O}_{2} \leftarrow \mathrm{O}+\mathrm{OH}$ & $0.1383 \times 10^{-1}$ & $0.1336 \times 10^{-1}$ \\
\hline $\mathrm{CH}_{2} \mathrm{O}+\mathrm{H} \rightarrow \mathrm{HCO}+\mathrm{H}_{2}$ & $0.1248 \times 10^{-1}$ & $0.1206 \times 10^{-1}$ \\
\hline $\mathrm{HCO}+\mathrm{H} \rightarrow \mathrm{CO}+\mathrm{H}_{2}$ & $0.1229 \times 10^{-1}$ & $0.1188 \times 10^{-1}$ \\
\hline
\end{tabular}

Table 5: Important reactions for the eigenvalue with positive real part for $n$ heptane ignition at time $5.25 \times 10^{-2}$ (sample \#151), which are also found important to methane and propane ignition. In all the above reactions $P_{\text {mode }_{i}}^{\bar{\omega}_{\tilde{\tau}}}=$ 0.9965 .

\begin{tabular}{|ccc|}
\multicolumn{4}{c}{ Eigenvalues with negative real parts. } \\
\hline Reaction & $\left|P_{\text {reaction }}^{\text {mode }_{k}}\right|$ & $\left|P_{\text {reaction }}^{\bar{\omega}}\right|$ \\
\hline $\mathrm{CH}_{4}+\mathrm{OH} \rightarrow \mathrm{CH}_{3}+\mathrm{H}_{2} \mathrm{O}$ & $0.5551 \times 10^{-1}$ & $0.5632 \times 10^{-3}$ \\
$\mathrm{CH}_{3}+\mathrm{O} \rightarrow \mathrm{CH}_{2} \mathrm{O}+\mathrm{H}$ & $0.5513 \times 10^{-1}$ & $0.5594 \times 10^{-3}$ \\
$\mathrm{C}_{2} \mathrm{H}_{4}+\mathrm{OH} \rightarrow \mathrm{C}_{2} \mathrm{H}_{3}+\mathrm{H}_{2} \mathrm{O}$ & $0.4016 \times 10^{-1}$ & $0.4074 \times 10^{-3}$ \\
$\mathrm{CH}_{4}+\mathrm{H} \leftarrow \mathrm{CH}_{3}+\mathrm{H}_{2}$ & $0.2106 \times 10^{-1}$ & $0.2137 \times 10^{-3}$ \\
$\mathrm{CH}_{4}+\mathrm{O} \rightarrow \mathrm{CH}_{3}+\mathrm{OH}$ & $0.1904 \times 10^{-1}$ & $0.1932 \times 10^{-3}$ \\
\hline
\end{tabular}

Table 6: Important reactions for the two eigenvalues with negative real parts for $\mathrm{n}$-heptane ignition at time $5.25 \times 10^{-2}$ (samples \#151), which are also found important to methane and propane ignition. In all the above reactions $P_{\text {mode }_{i}}^{\bar{\omega}_{\tilde{\tau}}}=$ $1.015 \times 10^{-2}$.

In Tables 7, 8 and 9 we listed the species which are affected the most by the time scales of the modes with a large contribution to the TSR $\left(P_{\text {mode }_{i}}^{\bar{\omega}_{\tilde{\tau}}}\right.$ greater than the tolerance). It can be seen that, for all the mechanisms, the species associated with the eigenvalue with positive real part are those connected with hydrogen chemistry $\left(\mathrm{H}, \mathrm{O}, \mathrm{O}_{2}\right.$ and $\left.\mathrm{OH}\right)$ and temperature. The $\mathrm{CH}_{4}$ species is only present in the case of methane kinetics. As far as the eigenvalues with negative real part are concerned, the most important species for methane are (in order of importance): $\mathrm{CH}_{3}, \mathrm{C}_{2} \mathrm{H}_{4}, \mathrm{CH}_{4}, \mathrm{C}_{2} \mathrm{H}_{6}, \mathrm{CH}_{2} \mathrm{O}, \mathrm{H}_{2}$ and $\mathrm{H}$. For the propane mechanism there are: $\mathrm{CH}_{4}, \mathrm{C}_{2} \mathrm{H}_{4}, \mathrm{H}$ and $\mathrm{H}_{2}$. Finally, for the n-heptane mechanisms we have: $\mathrm{CH}_{3} \mathrm{OH}, \mathrm{CH}_{4}, \mathrm{H}$ and $\mathrm{H}_{2}$.

From the inspection of the tables it can be seen that there is a pool of common species, reported in table 10, that is important for all the analyzed mechanisms. As already noted above, hydrogen chemistry is important for the eigenvalue with positive real part, while $\mathrm{CH}_{4}$ is the common species associated with the eigenvalue with negative real part.

\section{Non-normality and trajectory curvature}

We noted earlier that the TSR when computed considering the contribution of the direction cosines according with formula (31) might produce overshoots between the time evolution of
Eigenvalue with positive real part.

\begin{tabular}{|c|c|}
\hline Name & CSP pointer \\
\hline $\mathrm{H}$ & $3.62 \times 10^{-1}$ \\
\hline $\mathrm{O}$ & $1.46 \times 10^{-1}$ \\
\hline $\mathrm{O}_{2}$ & $1.88 \times 10^{-1}$ \\
\hline $\mathrm{OH}$ & $2.34 \times 10^{-1}$ \\
\hline $\mathrm{CH}_{4}$ & $1.82 \times 10^{-1}$ \\
\hline Temp & $3.77 \times 10^{-1}$ \\
\hline \multicolumn{2}{|c|}{ Eigenvalue with negative real part. } \\
\hline Name & CSP pointer \\
\hline $\mathrm{H}$ & $1.07 \times 10^{-1}$ \\
\hline $\mathrm{H}_{2}$ & $1.22 \times 10^{-1}$ \\
\hline $\mathrm{CH}_{4}$ & $3.91 \times 10^{-1}$ \\
\hline $\mathrm{C}_{2} \mathrm{H}_{4}$ & $6.71 \times 10^{-1}$ \\
\hline $\mathrm{CH}_{3}$ & $8.43 \times 10^{-1}$ \\
\hline $\mathrm{CH}_{2} \mathrm{O}$ & $2.22 \times 10^{-1}$ \\
\hline $\mathrm{C}_{2} \mathrm{H}_{6}$ & $2.41 \times 10^{-1}$ \\
\hline
\end{tabular}

Table 7: Species associated with the eigenvalues with positive and negative real parts for methane ignition at time 0.10701s (samples \#227).

Eigenvalue with positive real part.
\begin{tabular}{|c|c|}
\hline Name & CSP pointer \\
\hline $\mathrm{H}$ & $3.04 \times 10^{-1}$ \\
$\mathrm{O}$ & $1.39 \times 10^{-1}$ \\
$\mathrm{O}_{2}$ & $1.25 \times 10^{-1}$ \\
$\mathrm{OH}$ & $1.96 \times 10^{-1}$ \\
Temp & $4.88 \times 10^{-1}$ \\
\hline Eigenvalue with negative real part. \\
\hline Name & CSP pointer \\
\hline $\mathrm{H}$ & $1.81 \times 10^{-1}$ \\
$\mathrm{H}_{2}$ & $1.56 \times 10^{-1}$ \\
$\mathrm{C}_{2} \mathrm{H}_{4}$ & $1.66 \times 10^{-1}$ \\
$\mathrm{CH}_{4}$ & $3.26 \times 10^{-1}$ \\
\hline
\end{tabular}

Table 8: Species associated with the eigenvalues with positive and negative real parts for propane ignition at time $5.0994 \times 10^{-2} \mathrm{~s}$ (samples \#170).

the TSR and that of the eigenvalues. This happened for the subcritical regime of the Williams model (Fig. 18), and both in the super and subcritical regime of the Semenov model (Figs. 22 and 27).

It just occurs that even in more complicated problems such as those related to HC fuels, the evolution of the TSR when computed with Eq. (31) might develop overshoots between the time evolution of the TSR and that of the eigenvalues as noticed in the two dimensional model problems.

For example, consider again the oxidation of methane studied in Sec. 10.1. We will use the same dataset used to produce the results shown in Fig. 37 when the TSR is computed using Eq. (32). In this section however, the TSR is computed considering the direction cosine in the weights of Eq. (31). This different definition of the TSR causes the development of an overshoot while transiting from one scale to the next slower scale at about sample \#350 (see Fig. 47), while there is no overshoot in Fig. 37 when the TSR when is computed using Eq. (32). We verified that the two scales bracketing the TSR are both associated with eigenvalues with negative real parts.

We will show in this section that this peculiar evolution of the TSR is related to the near co-linearity of the eigenvectors associated with the two scales bracketing the oscillation of the 
Eigenvalue with positive real part.

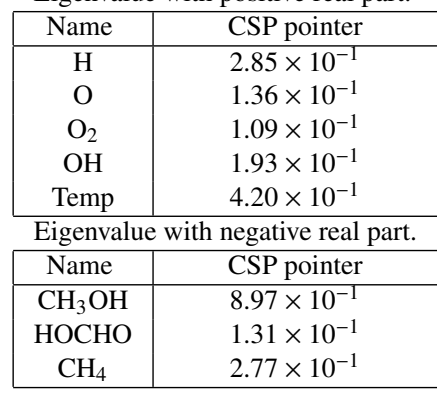

Table 9: Species associated with the eigenvalues with positive and negative real parts for n-heptane ignition at time $5.25 \times 10^{-2}$ (samples \#151).

Eigenvalue with positive real part.

\begin{tabular}{|c|c|}
\hline Name & CSP pointer \\
\hline $\mathrm{H}$ & $2.85 \times 10^{-1}$ \\
$\mathrm{O}$ & $1.36 \times 10^{-1}$ \\
$\mathrm{O}_{2}$ & $1.09 \times 10^{-1}$ \\
$\mathrm{OH}$ & $1.93 \times 10^{-1}$ \\
Temp & $4.20 \times 10^{-1}$ \\
\hline \multicolumn{2}{|c|}{ Eigenvalue with negative real part. } \\
\hline Name & CSP pointer \\
\hline $\mathrm{CH}_{4}$ & $2.77 \times 10^{-1}$ \\
\hline
\end{tabular}

Table 10: Species associated with the eigenvalues with positive and negative real parts for $n$-heptane ignition at time $5.25 \times 10^{-2}$ (samples \#151), which are also found important for methane and propane ignition.

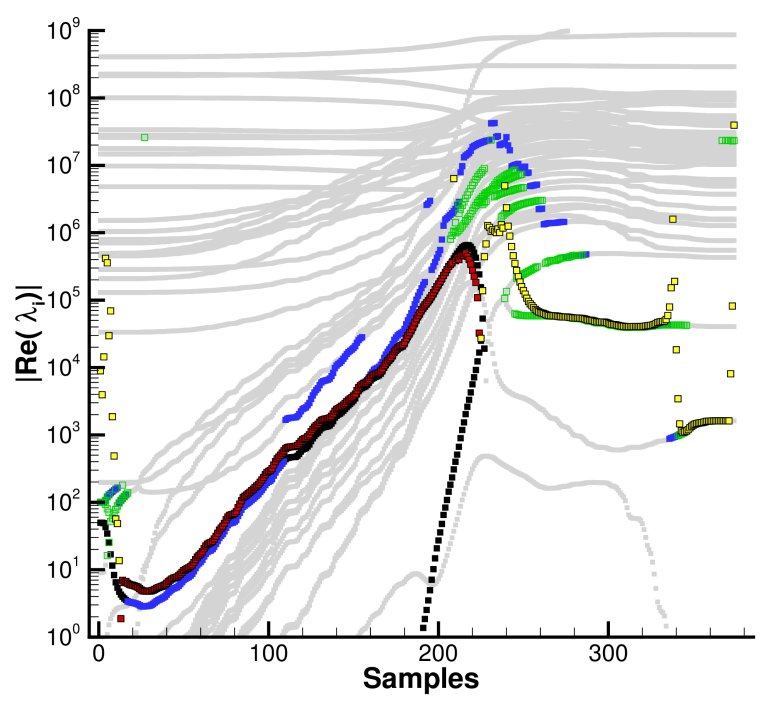

Figure 47: TSR analysis for methane. Eigenvalues with positive real parts (black markers); first non-exhausted mode $\lambda_{M+1}$ (blue markers); $\omega_{t}$ with positive sign (red markers), $\omega_{t}$ with negative sign (yellow markers), active subspace (green markers); $\omega_{\tau}$ computed considering the direction cosine in the weights (formula (31) exhibits an overshoot at about sample \#350.

TSR. In his seminal work on non-normal systems, Trefethen ([31], page 10) warns that "Eigenvalues do not always govern the transient behavior of a non-normal system", where a non- normal system can be defined as one involving matrices or operators $\mathrm{V}$, for which the inverse, if it exists, contains very large entries.

To assess whether non-normality could be the actual cause for the observed evolution of the TSR, we consider here a simple two-dimensional linear system with a tunable degree of nonnormality. To this end, we define two unit (column) vectors as:

$$
\boldsymbol{a}_{1}=\{\operatorname{Cos}[\gamma], \operatorname{Sin}[\gamma]\} \quad \boldsymbol{a}_{2}=\{\operatorname{Cos}[\gamma+\phi], \operatorname{Sin}[\gamma+\phi]\}
$$

where $\phi$ is a tunable angle comprised by the two vectors; define next a matrix $\mathrm{V}$ built upon $\boldsymbol{a}_{1}$ and $\boldsymbol{a}_{2}$ as:

$$
V=\left\{\begin{array}{ll}
a_{1} & a_{2}
\end{array}\right\}
$$

and its inverse $V^{-1}$; define two scales with a prescribed and constant spectral gap $\epsilon$ :

$$
\Lambda=\left\{-\epsilon^{-1},-1\right\}
$$

and, define a matrix $\mathbf{J}$ as:

$$
J=V \operatorname{diag}(\Lambda) V^{-1}
$$

As a measure of non-normality, we adopt the condition number of $\mathbf{J}$.

Define a linear Cauchy problem on the basis of the constant matrix J as:

$$
\partial_{t}\left\{\begin{array}{l}
y \\
z
\end{array}\right\}=J\left\{\begin{array}{l}
y \\
z
\end{array}\right\} \quad ; \quad\left\{\begin{array}{l}
y(0) \\
z(0)
\end{array}\right\}=\left\{\begin{array}{l}
y_{0} \\
z_{0}
\end{array}\right\}
$$

having a closed form analytic solution.

Figure 48 shows the trajectory obtained by integrating the set (55) with $\gamma=0.1(\pi / 2), \phi=0.25, \epsilon=10^{-2}, y_{0}=0, z_{0}=\phi$. With this selection of parameters, the Jacobian matrix is:

$$
J=\left(\begin{array}{ll}
-157.483 & 362.931 \\
-24.7844 & 56.4826
\end{array}\right)
$$

whose determinant is $\epsilon^{-1}=100$ (note that does not depend on the degree of co-linearity of the two eigenvectors, this being controlled by the value of $\phi$ ). Instead, the condition number of $\mathrm{J}$ depends on $\phi$, and, with the prescribed values of the model parameters, its value is 1603.24 , while the direction cosine between the two right eigenvectors is 0.968912 , both values denoting a rather high degree of non-normality.

Finally, we want to make a remark for the CSP users, which are familiar with the concept of CSP pointers. The two CSP pointer matrices associated with the two eigenvectors take the following values:

$$
\begin{aligned}
& \boldsymbol{a}_{1} \boldsymbol{b}^{1}=\left(\begin{array}{cc}
1.58063 & -3.66597 \\
0.250348 & -0.580633
\end{array}\right) \\
& \boldsymbol{a}_{2} \boldsymbol{b}^{2}=\left(\begin{array}{ll}
-0.580633 & 3.66597 \\
-0.250348 & 1.58063
\end{array}\right)
\end{aligned}
$$


The two matrices have diagonal entries larger than unity, and have rather large off diagonal terms. In this case, their usage as indicators to discriminate between major and minor (fast or slow) species is doubtful. Indeed, the co-linearity of the two eigenvectors, by construction, indicate that both modes contribute to the dynamics of the two species, and there is no way to attribute to one species or the other a different dynamical role for the prescribed set of parameters.
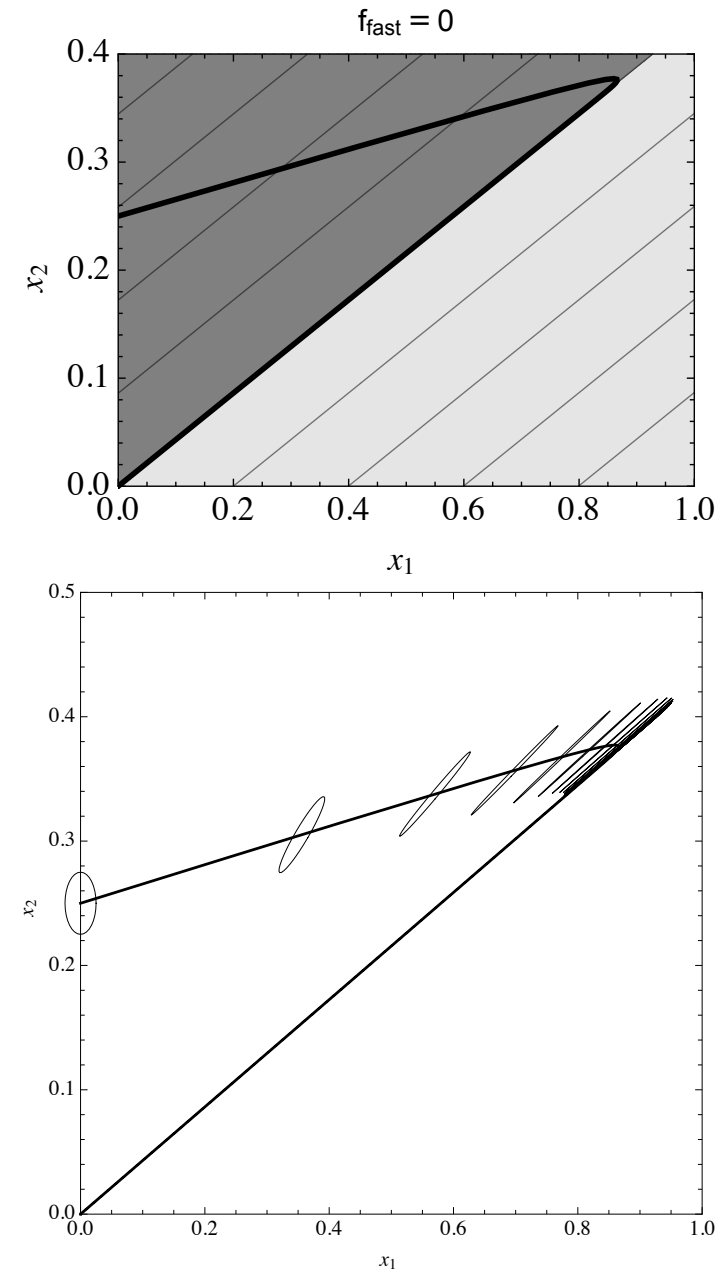

Figure 48: Evolution of the trajectory and of the circular probe in the phase space, with overshoots $\left(y_{0}=0, z_{0}=\phi\right)$; (top) isocontour lines refer to constant fast eigenvalue magnitude.

Fig. 48 shows that the trajectory emanating from the initial conditions $\left(y_{0}=0, z_{0}=\phi\right)$ proceeds first along the direction of the fast negative eigenvector $\boldsymbol{a}_{1}$ at the pace $-\epsilon^{-1}=-100$. The circular probe experiences a contraction along the direction $\boldsymbol{a}_{1}$, but also a rotation caused by the off-diagonal term of the Jacobian (non-normality). The rotation deforms the circular probe into an ellipsoidal shape having the largest axis nearly parallel to the slow eigenvector $\boldsymbol{a}_{2}$. There exists a small zone were the trajectory turns from the fast to the slow directions. There, both scales are equally important. At the time that the trajectory becomes essentially parallel to the slow direction $\boldsymbol{a}_{2}$, the ellipsoidal shape degenerated into an essentially one dimensional closed line. This closed shape keeps being shrunken at the slow pace -1 while it proceeds along the SIM defined by the algebraic constraint $f^{1}=\boldsymbol{b}^{1} \cdot \boldsymbol{g}=0$, until it reaches the fixed point $(0,0)$.

The time evolution of the TSR is plotted in Fig. 49 with weights computed with formula (31) and (32), together with the, constant, values of the fast, $-\epsilon^{-1}$, and slow scales, -1 .

In Fig. 49, the TSR with weights computed with formula (31) (long dashed line) exhibits the same qualitative evolution observed in Fig. 47 for the methane system, that is (i) initially TSR follows closely the fast scale $-\epsilon^{-1}$ in the initial transient, (ii) next, it attains values much smaller than $-\epsilon^{-1}$, soon later (iii) much larger than the slow scale -1 (even positive!), and eventually, (iv) it closely follows the slow scale set by -1 during the final approach to the fixed point.

Note instead that that TSR with weights computed with formula (32) (dashed black line) proceeds monotonously from the fast scale $-\epsilon^{-1}$ to the slow scale -1 without developing any overshoot.

Since the only difference in the two formulation is the presence or absence of the director cosines, we can attribute the overshoot to this term, which plays a significant role when the system is non-normal.

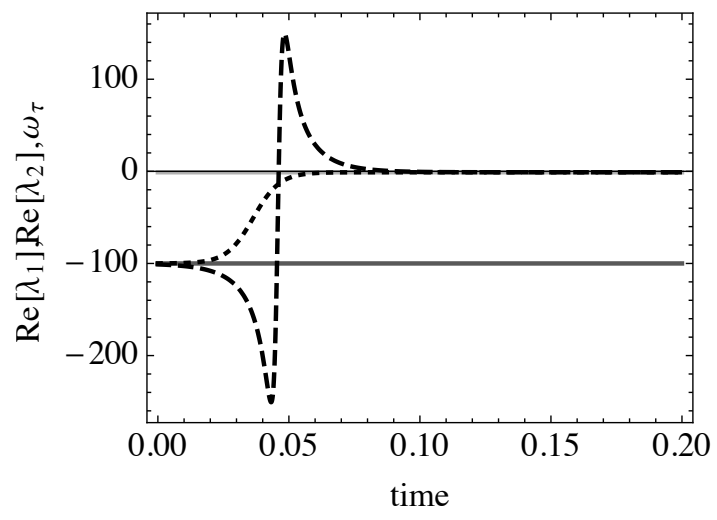

Figure 49: Time evolution of the TSR with overshoots $\left(y_{0}=0, z_{0}=\phi\right)$; real part of eigenvalues (dark gray lines); TSR computed according with formula (31) (long dashed black line) and (32) (dashed black line).

However, having nearly co-linear eigenvectors is only a necessary condition to observe the time evolution depicted in Fig. 49. A further condition is related to the selection of the initial condition. Indeed, Figure 50 shows the trajectory obtained by integrating the set (55) with the same set of parameters, but with a new initial condition: $y_{0}=2, z_{0}=\phi$. The condition number of $\mathbf{J}$ is clearly identical for the two tests, since $J$ is the same for both cases.

The time evolution of the TSR obtained in this second test is plotted in Fig. 51. It is apparent that although the set of parameters is unchanged, the different initial condition selects a trajectory along which the TSR computed with formula (31) (long dashed line) does not exhibit the overshoots; indeed, in this case TSR monotonously evolves from $-\epsilon^{-1}$ to -1 .

The main difference of the two cases consists in the presence 

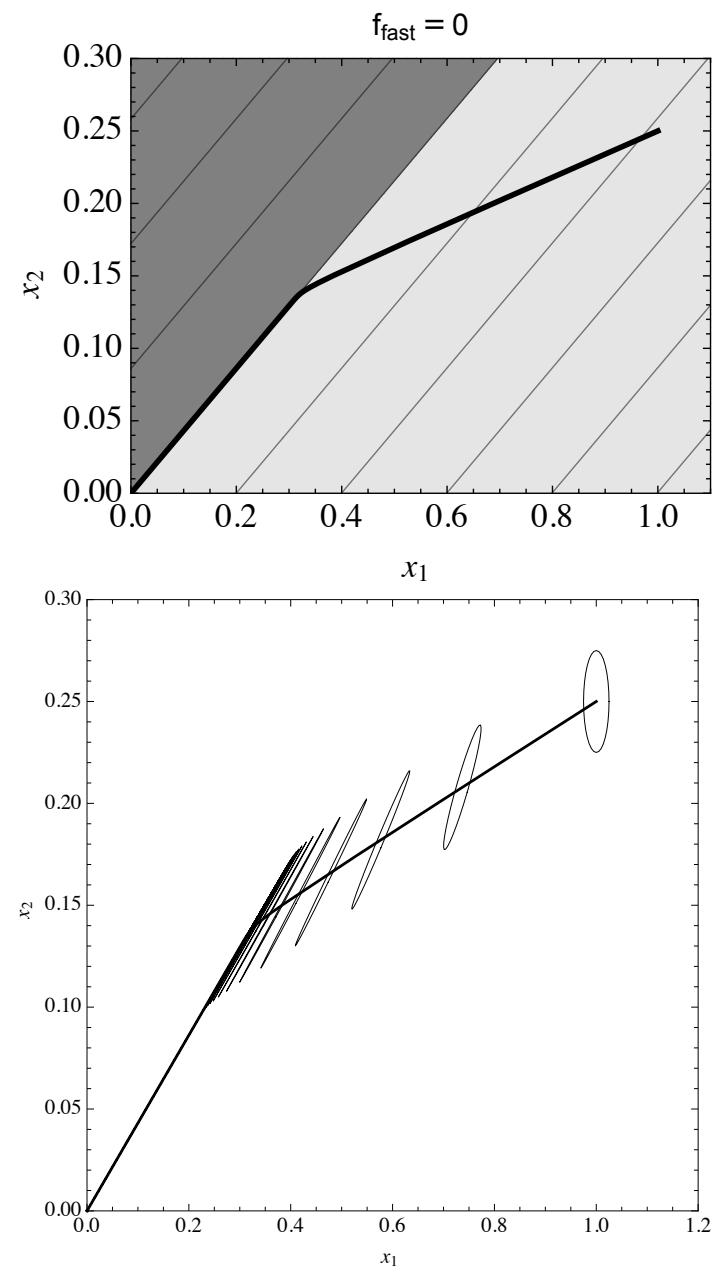

Figure 50: (top) isocontour lines refer to constant fast eigenvalue magnitude; (bottom) evolution of the trajectory and of the circular probe in the phase space with no overshoots $\left(y_{0}=2, z_{0}=\phi\right)$.

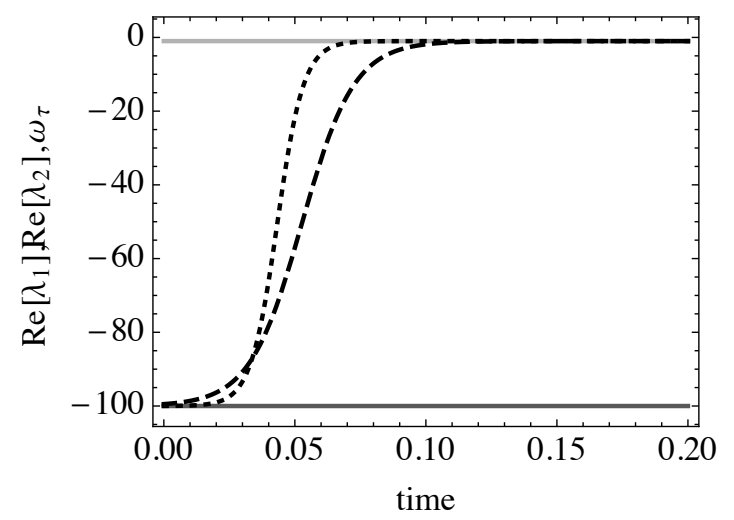

Figure 51: Time evolution of the TSR with no overshoots $\left(y_{0}=2, z_{0}=\phi\right)$; real part of eigenvalues (dark/light gray lines); TSR computed according with formula (31) (long dashed black line) and (32) (dashed black line).

or absence of the development of a strong curvature of the trajectory in the phase space (compare Fig. 48 to Fig. 50), while transitioning from one scale to the next. Therefore, the develop- ment of the overshoots can be attributed to the strong curvature of the trajectory.

We can thus conclude that the TSR computed with with Eq. (31) is able to sense the curvature of the trajectory as measured by the direction cosine between eigenvectors and the vector field $\boldsymbol{g}$, whereas the TSR computed with with Eq. (32) is not able to sense this dynamical feature.

\section{Discussion and Conclusions}

The Tangential Stretching Rate concept, combined with the local decomposition of the Jacobian matrix, provides information about the most energetic scale and the range of active scales.

The definition of the Tangential Stretching Rate combines three aspects of the dynamics, that turn out to be equally important in the generation of the most energetic scale:

(1) the eigenvalues, $\lambda_{i}$, of the Jacobian matrix, $J$, of the local vector field, $\boldsymbol{g}$, of the system;

(2) the normalized amplitude, $f^{i}=\boldsymbol{b}^{i} \cdot \boldsymbol{g}$, of each eigenmode $a_{i}$;

(3) the degree of co-linearity, $\boldsymbol{g} \cdot \boldsymbol{a}_{i}$, of each eigenvector $\boldsymbol{a}_{i}$ with respect to the vector field $\boldsymbol{g}$.

The Tangential Stretching Rate is a weighted average of all eigenvalues in (1) with weights that depend on both (2) and (3). Each mode can in principle contribute to the TSR providing its mode amplitude is large (high energy), and its direction sufficiently aligned with that of the vector field.

The analysis here presented indicates that the proper characteristic chemical time scale in problems involving multi-step chemical kinetic mechanisms is $\tau_{\text {chem }}:=\left|1 / \bar{\omega}_{\tilde{\tau}}\right|$, and it can be used to define a Damköeler number, $D a$, in conjunction with a characteristic fluid dynamical scale $\tau_{f}$ to yield $D a:=\tau_{f} / \tau_{\text {chem }}$. This conclusion can be drawn by observing that $\tau_{\text {chem }}:=\left|1 / \bar{\omega}_{\tilde{\tau}}\right|$ (i) is the most relevant time scale during both the explosive and relaxation regimes, (ii) is intrinsic to the dynamics, and, as such, can be identified without the need of any ad-hoc assumption.

In this regard, it is worth comparing the concept of the TSR with that of chemical explosive mode $(\mathrm{CEM})$, the latter introduced by $\mathrm{Lu}$, et al. in [7], and defined as $\tau_{C E M}:=\left|1 / \lambda_{a+}\right|$, where $\lambda_{a+}$ is the fastest eigenvalue with positive real part in the system, as follows:

1. the TSR recovers the CEM scale when $W_{a+}=1$ and $W_{i \neq a+}=0$, that is, when mode $i=a+$ has unit weight (which implies co-linearity with the vector field), and all modes $i \neq a+$ have zero amplitude.

2. Even when the eigenvalue with positive real part in the system, $\lambda_{a+}$, is the sole participant to the TSR, it can happen that $W_{a+} \ll 1$. In fact, it suffices that the amplitude of mode $a+$ be small, $f^{a+} \sim 0$, to yield a small weight $\bar{W}_{a+}=\left(f^{a+} / \mathrm{g}\right)^{2} \sim 0$, and in turn a small TSR value, $\bar{\omega}_{\tilde{\tau}}=\bar{W}_{a+}\left|\lambda_{a+}\right| \sim 0$. What this means is that the presence of an eigenvalue with positive real part in the system 
is a necessary but not sufficient condition for ignition. Ignition requires that the corresponding mode amplitude be also large. In other words, the eigenvalue with positive real part is a potential route for ignition: the actual ignition requires enough energy as measured by the mode amplitude. This statement is especially noteworthy when any of the above criteria is used to post-process computed reactive flows.

3. A similar conclusion has been drawn by Boivin, et al. [14], page 1787 , in their analysis of a supersonic hydrogen/air autoignition-stabilized flame. Boivin states that the eigenvalue with positive real part marks the mixture "reactivity", but not the actual occurrence of ignition. Their analysis concludes that another variable, the chemical steadystate parameter defined as $\alpha=$ (production rate $\left(\mathrm{HO}_{2}\right)$ - destruction rate $\left.\left(\mathrm{HO}_{2}\right)\right)$ /production rate $\left(\mathrm{HO}_{2}\right)$, was required to mark the actual ignition progress. Indeed, our TSR analysis shows (Fig. 40) that the species most contributing to the magnitude of the TSR during the initiation regime are $\mathrm{HO}_{2}, \mathrm{H}_{2} \mathrm{O}_{2}, \mathrm{CH}_{2}$, and $\mathrm{CH}_{2} \mathrm{O}$, a list which includes $\mathrm{HO}_{2}$. Thus, both our analysis and Boivin's point out that the actual inception of ignition requires both a "channel" (a positive eigenvalue) and a "content", this being perceived either by a large mode amplitude, or by a large Boivin's chemical steady-state parameter.

4. Boivin's methodology aims at producing an analytical approximation of the eigenvalue with positive real part; however, to apply their method requires the preliminary identification of the reactions most affecting ignition. The discussion above should make clear that the TSR and the Participation Index to the TSR are ideal candidates to complement Boivin's approach.

5. As stated in the previous comments, the CEM scale might not coincide with the TSR when more modes contribute to the TSR, when $\boldsymbol{a}_{a+}$ is not co-linear with the vector field, and when the mode amplitude of the fast positive eigenvalue is small. When any of the above conditions apply, the relevance of the CEM is questionable.

6. the TSR is defined at all time and locations, whereas CEM is not defined when and where a positive eigenvalue does not exist.

7. Finally, the TSR can be applied to systems of PDEs after, say, having discretized the right hand side according with the method of lines. In this circumstance, there might coexist very many positive eigenvalues, and the TSR will be still able to identify the current most energetic time scale and the processes contributing to it. This problem cannot be dealt by CEMA, because CEMA requires an a-priori ansatz to pick the proper positive eigenvalue.

We can also establish the connection with the scales introduced in the CSP method and TSR. The time scale associated with the fastest of the energy containing scales, $\tau_{L+1}:=\left|1 / \lambda_{L+1}\right|$ finds is upper bound in the $\tau_{M+1}$ scale identified in CSP as the fastest scale of the "slow" modes. On the other hand, the most significant progress of the TSR with respect to the classic CSP methodology is the possibility to identify the most energy containing scale, which is a missing concept in CSP. From this perspective the TSR parameter is not complementing and not competing with the CSP analysis.

Finally, this paper introduced three nonlinear planar models of auto-ignition. The analysis of these prototypical models allowed us to investigate the role of the nonlinearities in branched-chain reactions (polynomial) and non-isothermal (exponential) systems and their combined action. We found that ignition by non-isothermal branched-chain reactions, differently from isothermal branched-chain reactions, might initiate with a pair of real eigenvalues having different sign and also that the transition from positive to negative sign can occur without crossing a region of complex eigenvalues.

The planar linear model allowed us to study the role of nonnormality in kinetic systems, which might result in overshoots of the TSR index associated with the presence of a strong curvature of the trajectory in the phase space.

The TSR method has been applied to the study of the autoignition of three different fuels: methane, propane and nheptane. The analysis applied to these complex kinetic mechanisms indicates that the most energy containing scale $\bar{\omega}_{\tilde{\tau}}$ tracks at first the fast positive eigenvalue and then, before the merging, it feels the influence of the dissipative modes. This behavior is qualitatively very similar for all the mechanisms and the maximum value of $\bar{\omega}_{\tilde{\tau}}$ are of similar magnitude. The analysis of the important indices enables to identify the most important reactions common to all mechanisms.

In closing, we remark that in all the test cases discussed in this work, the identification of the explosive time scales in the autoignition problems treated (see Figs. 39, 43 and 44) indicates that these time scales are (almost always) much slower than the fastest time scales in the dynamics of the problem because all other faster time scales become exhausted. It is specifically on the exploitation of this feature that the traditional methodology (asymptotics) to identify the explosive time scales involved first the construction of a reduced model, and next the analysis of the dominant dynamics of the reduced model by identifying the reactions in the reduced model that were responsible the most for the generation of the explosive time scale. A significant novelty of the algorithm proposed here is that it can identify the characteristic time scale without the explicit need to construct a reduced model.

\section{Acknowledgements}

MV and EM acknowledge the support of the Italian Ministry of University and Research (MIUR). MV acknowledges the support of the CCRC at KAUST for the development of the CSPTk library. The authors are thankful to C.Safta for their support during the harmonization of TChem with CSPTk.

\section{References}

[1] A. Kazakov, M. Chaos, Z. Zhao, and F.L. Dryer, J. Phys. Chem. A, 110 (2006) 7003-7009. 
[2] D. Diamantis, D. Kyritsis, D.A. Goussis, Two Stage Ignition of n-heptane: Identifying the Chemistry Setting the Explosive Time Scales, 2nd Intl. Conference in Model Reduction in Reacting Flows, South Bent, Indiana, USA, April 2009.

[3] D.J. Diamantis, D.C. Kyritsis, D.A. Goussis, Proc. Combustion Institute, 35, (2015) 267-274.

[4] Z. Chen, X. Qin, Y. Ju, Z. Zhao, M. Chaos, F.L. Dryer, Proc. Combustion Institute, 31, (2007) 1215-1222.

[5] S. Gupta, H.G. Im , M. Valorani, Proc. Combustion Institute, 33 (2), (2011) 2991-2999.

[6] S. Gupta, H.G. Im , M. Valorani, Proc. Combustion Institute, 34 (2013) 1125-1133.

[7] T. Lu, C.S. Yoo, J.H. Chen, and C.K. Law, J. Fluid Mech., 652 (2010) 45-64.

[8] C.S. Yoo, T. Lu, J.H. Chen, and C.K. Law, Combust. Flame, 158, (2011) 1727-1741.

[9] Z. Luo, C.S. Yoo, E.S. Richardson, J.H. Chen, C.K. Law, and T. Lu, Combust. Flame, 159 (2012) 265-274.

[10] R. Shan, C.S. Yoo, J.H. Chen, and T. Lu, Combust. Flame, 159 (2012) 3119-3127.

[11] R. Shan, T. Lu, Combust. Flame, 161 (7) (2014) 1716-1723.

[12] H.N. Najm, M. Valorani, and D.A. Goussis, Combust. Theory Modelling, 14 (2010) 257-294.

[13] A. Adrover, F. Creta, M. Giona, and M. Valorani, J.Comput. Phys., 225 (007) 1442-1471.

[14] P. Boivin, A. Dauptain, C. Jimenez, B. Cuenot, Combust. Flame, 159, (2012) 1779-1790.

[15] R.J. Kee, F.M. Rupley, E. Meeks, J.A. Miller, Chemkin-III: a Fortran chemical kinetics package for the analysis of gas-phase chemical and plasma kinetics, UC-405, SNAD96-8216, May 1996.

[16] C. Safta, H.N. Najm, O. Knio, TChem - A Software Toolkit for the Analysis of Complex Kinetic Models, SANDIA REPORT, SAND2011-3282, Unlimited Release, May 2011.

[17] P.N. Brown, G.D. Byrne, and A.C. Hindmarsh, SIAM J. Sci. Stat. Comput., 10 (1989) 1038-1051.

[18] G.P. Smith, D.M. Golden, M. Frenklach, N.W. Moriarty, B. Eiteneer, M. Goldenberg, C.T. Bowman, R.K. Hanson, S. Song, W.C. Gardiner, Jr., V.V. Lissianski, and Z. Qin, GRI-Mech 3.0, www.me.berkeley.edu/gri mech, Version:3.0, 7/30/99.

[19] H.J. Curran, P. Gaffuri, W.J. Pitz, and C.K. Westbrook, Combust. Flame, 114 (1998) 149-177.

[20] F.A. Williams, Combustion Theory, Westview Press, $2^{\text {nd }}$ Edition, 570-573.

[21] F. Creta, A. Adrover, S. Cerbelli, M. Valorani, and M. Giona, J. Phys. Chem. A, 110 (2006) 13447-13462.

[22] A. Kapila, J. Engineering Mathematics, 12 (3), July 1978.

[23] M. Valorani, and S. Paolucci, J.Comput. Phys., 228 (2009) 46654701.

[24] M. Valorani, H.N. Najm, and D.A. Goussis, Combust. Flame, 134 (2003) 35-53.

[25] A. Adrover, S. Cerbelli, and M.Giona, Int. J. Bifurc. Chaos, 12 (2002) 353-368.

[26] A. Lichnerowitz, Linear Algebra and Analysis, Holden-Day,San Francisco, 1967.

[27] M. Giona, A. Adrover, F. Creta, and M. Valorani, J. Phys. Chem. A, 110 (2006) 13463-13474.

[28] K.D. Mease, S. Bharadwaj, and S. Iravanchy, Control and Dynamics, 26 (2003) 318-330.

[29] C.K. Law, Combustion Physics, Cambridge University Press, 2006.

[30] L. Perko, Differential Equations and Dynamical Systems, Springer, 2001.

[31] L.N. Trefethen, M. Embree, Spectra and Pseudospectra, Princeton University Press, 2005

[32] A. Adrover, F. Creta, S. Cerbelli, M. Valorani, M. Giona, Computers and Chemical Engineering, 31 (2007) 1456-1474.

[33] N.N. Semenov, Zur theorie des verbrennungprozesses. Zeitschrift fur Physics, 48 (1928) 571-581.

[34] N.N. Semenov (1959). Some problems of chemical kinetics and reactivity. London: Pergamon Press.

[35] H.N. Najm, M Valorani, Journal of Computational Physics, 270 (2014), 544-569.

\section{Appendix A. Complex Eigenvalues}

We noted in Fig. 8 that the TSR takes very large values (in modulus) when there are complex eigenvalues. The source of these large values can be explained if we decompose the Jacobian matrix. Before doing this, it is worth discussing how to carry out the matrix decomposition (and algorithmic issues) when complex eigenvectors are present.

Consider the linear system

$$
\dot{\boldsymbol{x}}=A \cdot \boldsymbol{x},
$$

for which the following theorem holds [30]:

Theorem: If the $2 n \times 2 n$ real matrix $A$ has $2 n$ distinct complex eigenvalues $\lambda_{j}=a_{j} \pm i b_{j}$ and corresponding complex eigenvectors $\mathbf{w}_{j}=\boldsymbol{u}_{j} \pm i \boldsymbol{v}_{j}, j=1, \ldots, n$, then $\boldsymbol{u}_{1}, \boldsymbol{v}_{1}, \ldots, \boldsymbol{u}_{n}, \boldsymbol{v}_{n}$ is a basis for $\mathbf{R}^{2 n}$, the matrix

$$
Q=\left[\boldsymbol{u}_{1}, \boldsymbol{v}_{1}, \boldsymbol{u}_{2}, \boldsymbol{v}_{2}, \ldots, \boldsymbol{u}_{n}, \boldsymbol{v}_{n}\right]
$$

is invertible, and

$$
Q^{-1} \cdot A \cdot Q=\operatorname{diag}\left(\begin{array}{cc}
a_{J} & b_{J} \\
-b_{J} & a_{J}
\end{array}\right)
$$

is a real $2 n \times 2 n$ matrix with $2 \times 2$ blocks along the diagonal.

Now, using this method in order to build the matrices of right and left eigenvectors, we write $J_{g}=A \cdot \Lambda \cdot B$, where the matrix $\Lambda$ is no longer diagonal, but is a real matrix with $m 2 \times 2$ blocks along the diagonal (with $m$ number of pairs of complex eigevalues). Because of Eq. (A.3), Eq. (18) must be modified as follows:

$$
\begin{aligned}
\omega_{\tilde{\boldsymbol{\tau}}} & =\tilde{\boldsymbol{\tau}} \cdot J_{\boldsymbol{g}} \cdot \tilde{\boldsymbol{\tau}} \\
& =\frac{1}{\mathrm{~g}^{2}}(\boldsymbol{g} \cdot A \cdot \Lambda \cdot B \cdot \boldsymbol{g}) \\
& =\frac{\boldsymbol{g}}{\mathrm{g}^{2}} \cdot \sum_{i, j=1}^{N} \boldsymbol{a}_{j} \Lambda_{i}^{j}\left(\boldsymbol{b}^{i} \cdot \boldsymbol{g}\right) \\
& =\frac{\boldsymbol{g}}{\mathrm{g}^{2}} \cdot \sum_{i, j=1}^{N} \boldsymbol{a}_{j} \Lambda_{i}^{j} f^{i} \\
& =\frac{1}{\mathrm{~g}^{2}} \sum_{i, j=1}^{N}\left(\boldsymbol{g} \cdot \boldsymbol{a}_{j}\right) \Lambda_{i}^{j} f^{i} .
\end{aligned}
$$

Expanding $\boldsymbol{g}$ in eigenmodes through Eq. (19), one obtains

$$
\omega_{\tilde{\tau}}=\sum_{i, j=1}^{N}\left(\frac{f^{i}}{\mathrm{~g}^{2}} \sum_{k=1}^{N} f^{k}\left(\boldsymbol{a}_{k} \cdot \boldsymbol{a}_{j}\right)\right) \Lambda_{i}^{j}=\sum_{i, j=1}^{N} W_{j}^{i} \Lambda_{i}^{j}
$$

with

$$
W_{j}^{i}:=\frac{f^{i}}{\mathrm{~g}} \sum_{k=1}^{N} \frac{f^{k}}{\mathrm{~g}}\left(\boldsymbol{a}_{k} \cdot \boldsymbol{a}_{j}\right) .
$$

With this decomposition, it is now possible to see which terms are responsible for the behavior of the TSR when the eigenvectors are complex. It has been shown indeed, that where there is a pair of complex eigenvalues, $\omega_{\tilde{\tau}}$ is characterized by 


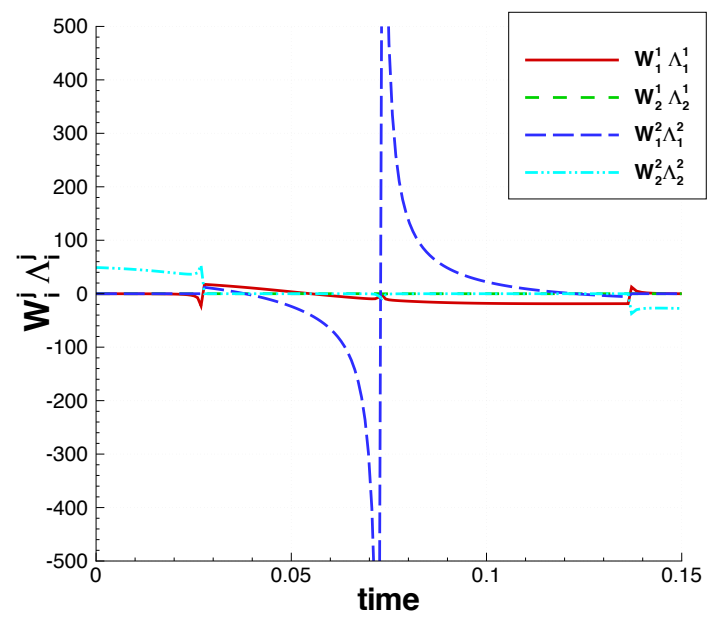

Figure A.1: Time evolution of $W_{j}^{i} \Lambda_{i}^{j}$ terms in TSR.

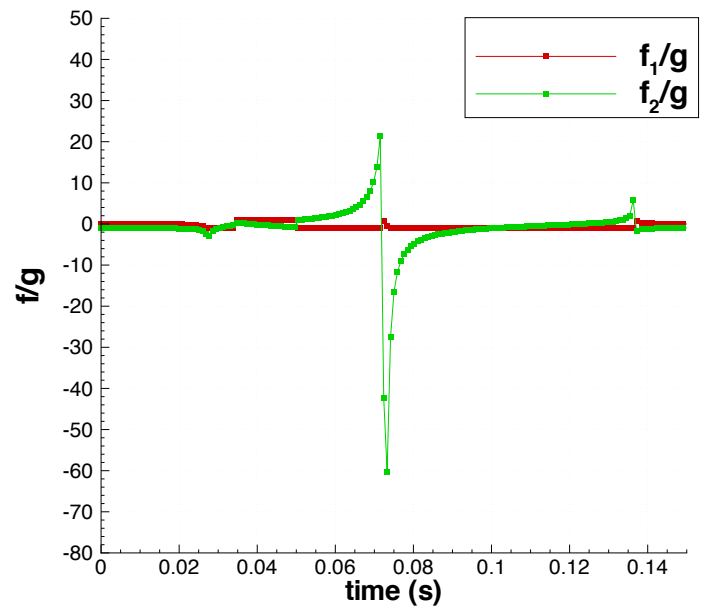

Figure A.2: Time evolution of $f / g$ terms in TSR

very high (in modulus) values. Figure A.1 shows the time evolution of each of the terms $W_{j}^{i} \Lambda_{i}^{j}$ in Eq. (A.5). In the William's model there are four of such terms; we found that the one responsible for the divergence of the TSR is the off diagonal term $W_{1}^{2} \Lambda_{1}^{2}$ (rotational term). In turn $W_{1}^{2}$ depends on $f^{2} / g$ term is characterized by two opposite peaks (Fig. A.2). 